\title{
Pregnancy after caesarean : current care, clinical prediction and risk counselling
}

Citation for published version (APA):

Schoorel, E. N. C. (2015). Pregnancy after caesarean : current care, clinical prediction and risk counselling. [Doctoral Thesis, Maastricht University]. Uitgeverij BOXPress. https://doi.org/10.26481/dis.20150623es

Document status and date:

Published: 01/01/2015

DOI:

10.26481/dis.20150623es

Document Version:

Publisher's PDF, also known as Version of record

\section{Please check the document version of this publication:}

- A submitted manuscript is the version of the article upon submission and before peer-review. There can be important differences between the submitted version and the official published version of record.

People interested in the research are advised to contact the author for the final version of the publication, or visit the DOI to the publisher's website.

- The final author version and the galley proof are versions of the publication after peer review.

- The final published version features the final layout of the paper including the volume, issue and page numbers.

Link to publication

\footnotetext{
General rights rights.

- You may freely distribute the URL identifying the publication in the public portal. please follow below link for the End User Agreement:

www.umlib.nl/taverne-license

Take down policy

If you believe that this document breaches copyright please contact us at:

repository@maastrichtuniversity.nl

providing details and we will investigate your claim.
}

Copyright and moral rights for the publications made accessible in the public portal are retained by the authors and/or other copyright owners and it is a condition of accessing publications that users recognise and abide by the legal requirements associated with these

- Users may download and print one copy of any publication from the public portal for the purpose of private study or research.

- You may not further distribute the material or use it for any profit-making activity or commercial gain

If the publication is distributed under the terms of Article $25 \mathrm{fa}$ of the Dutch Copyright Act, indicated by the "Taverne" license above, 


\section{Pregnancy after caesarean}

Current care, Clinical prediction and Risk counselling 
Thesis Universiteit Maastricht

ISBN 978-94-6295-188-4

(C) E.N.C Schoorel, Maastricht 2015

Cover design: Mandy Bronsgeest, ORANJEBRUIN grafisch ontwerp

Lay-out: Tiny Wouters

Printed by Proefschriftmaken.nl || Uitgeverij BOXPress, 's-Hertogenbosch

This research was funded by the Netherlands Organisation for Health Research and Development (ZonMW grant No 17100.3006).

Financial support by the SBOH for the publication of this thesis is gratefully acknowledged.

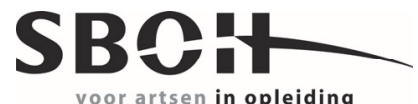

Additional financial support for the publication of this thesis by ABN Amro is gratefully acknowledged. 


\title{
Pregnancy after caesarean
}

\section{Current care, Clinical prediction and Risk counselling}

\author{
PROEFSCHRIFT \\ ter verkrijging van de graad van doctor \\ aan de Universiteit Maastricht, \\ op gezag van de Rector Magnificus, Prof. dr. L.L.G. Soete, \\ volgens het besluit van het College van Decanen, \\ in het openbaar te verdedigen op \\ dinsdag 23 juni 2015 om 12.00 uur \\ door
}

Ellen Nynke Caroliene Schoorel 


\section{Promotor}

Prof. dr. J.G. Nijhuis

\section{Copromotoren}

Dr. R.P.M.G. Hermens, IQ healthcare Radboud Universitair Medisch Centrum Dr. H.C.J. Scheepers

\section{Beoordelingscommissie}

Prof. dr. M.H. Prins, voorzitter

Dr. M.J. Faber, IQ healthcare Radboud Universitair Medisch Centrum

Prof. dr. S.A. Scherjon, Universitair Medisch Centrum Groningen

Prof. dr. M.E.A. Spaanderman

Prof. dr. R.G. de Vries, Academie Verloskunde Maastricht 


\section{Contents}

$\begin{array}{lll}\text { Chapter } 1 \text { General introduction. } & 7\end{array}$

Part I Current practice of caesarean sections in The Netherlands.

Chapter 2 SIMPLE: Implementation of recommendations from international evidence-based guidelines on caesarean sections in the Netherlands. Protocol for a controlled before and after study.

Chapter 3 Development and measurement of guidelines-based quality indicators 35 of caesarean section care in the Netherlands: a RAND-modified Delphi procedure and retrospective medical chart review.

Chapter 4 Practice variation of vaginal birth after cesarean: a registration based cohort study.

Part II Improving current practice on pregnancy following caesarean section.

Chapter 5 Predicting successful intended vaginal delivery after previous caesarean section: external validation of two predictive models in a Dutch nationwide registration-based cohort with a high intended vaginal delivery rate.

Chapter 6 Vaginal birth after a caesarean section: the development of a Western European population based prediction model for deliveries at term.

Chapter 7 Probability of vaginal birth after caesarean: do not ask the obstetrician.

Chapter 8 Involving women in personalised decision-making on mode of delivery after caesarean section: the development and pilot testing of a patient decision aid.

Chapter 9 Summary and General discussion.

Valorisatie.

Nederlandstalige samenvatting.

Dankwoord.

Curriculum Vitae. 



\section{Chapter 1}

\section{General introduction}

Adapted from:

ENC Schoorel, HCJ Scheepers, JG Nijhuis

Pregnancy and delivery after prior caesarean section: shared decision making and be aware of signs of

(imminent) uterine rupture

Nederlands Tijdschrift voor Obstetrie en Gynaecologie 2012;125:384-389 


\section{General introduction}

The caesarean section (CS) was originally designed as an emergency life-saving procedure for the pregnant woman. However, the nowadays worldwide rising CS rates implicate unnecessary use of this procedure which might even lead to higher morbidity and costs. The increasing CS rates also result in an expanding group of women who are pregnant with a CS scar, which demands a different approach to the management of pregnancy and labour. Clear evidence-based peer-reviewed guidelines exist on when to perform a CS, however to what extent these guidelines are implemented is currently unknown. This thesis will focus on the analysis of guideline-adherence around the performance of CSs in The Netherlands (part one). Subsequently a tailored strategy to improve care will be developed (part two) which is focused on management of birth after CS. In this chapter, an introduction is provided to current practice of CSs. Also, in respect to part two of this thesis, management of pregnancy after CS will be discussed in particular. The chapter concludes with a description of the aims an outline of this thesis.

\section{Part I Current practice of caesarean sections}

In 1985, the WHO advised a CS rate of 10 to $15 \% .{ }^{1}$ Yet, internationally, the rate of CSs has risen in many countries far beyond this recommended range. The highest reported rates are $41.9 \%$ in Iran and the Dominican Republic to $45.9 \%$ in Brazil. ${ }^{2}$ In The Netherlands, the CS rate has risen from $7.4 \%$ to $16.4 \%$ between 1990 and 2013.3.4 A study amongst 22 industrialized countries showed that between 1987 and 2007, 20 of the 22 countries had a statistical significant rise of their CS rate, with a mean relative increase of $49 \% .^{5}$ This rise in CSs implies not only a higher risk of feto-maternal complications ${ }^{6,7}$ but also enormous economic implications. A study performed by the WHO showed that in 2008, the costs of all CSs exceeding the level of $15 \%$ are 2.32 billion US dollars based on data of 137 countries, representing $95 \%$ of global births per year. ${ }^{8}$

\section{Implementation of contemporary guidelines}

Compared to a planned vaginal delivery, women with a planned CS have a higher risk of prolonged hospital stay, hysterectomy and cardiac arrest. ${ }^{9}$ For the child there is an increased risk of admission to the neonatal intensive care unit. ${ }^{9}$ Yet a planned CS reduces the risk of perineal and abdominal pain during birth and three days postpartum, early postpartum haemorrhage and obstetric shock. ${ }^{9}$ Hence, clear evidence-based recommendations on when to perform a CS are provided in several national and international guidelines such as the NICE guideline of the Royal College of Obstetricians and Gynaecologists (RCOG) ${ }^{9}$ and 
similar guidelines provided by the American College of Obstetricians and Gynaecologists $(\mathrm{ACOG})^{10}$ and the Dutch society of Obstetrics and Gynaecology (NVOG). ${ }^{11}$ Leading indications for CSs include failure to progress during labour (35\%), non-reassuring fetal heart rate pattern (24\%) and fetal malpresentation (19\%). ${ }^{12}$ To what extent contemporary guideline recommendations are implemented is currently unknown. We hypothesize that one of the causes of the rising CS rates might be inadequate adherence to these guidelines. This is supported by the finding of a meta-analysis of guideline implementation strategies of practice of CSs which showed that CS rates can be reduced without impeding safety for mother and child using interventions on guideline adherence. ${ }^{13}$ Interventions which are thoroughly designed and correctly targeted might lead to a change of approximately $5-10 \% .{ }^{14,15}$ Chaillet et al. ${ }^{16}$ have shown in a meta-analysis of guideline implementations studies within obstetrics, that in this field educational strategies are generally ineffective. Yet a multifaceted strategy, incorporating feedback from audits and supported by the organisation, were much more effective. The authors recommended to tailor the implementation strategy to the target-group by carrying out a prospective study which sets out to identify possible barriers to change. This approach is reflected in an evidence-based stepwise approach for implementing evidence-based guidelines which was developed by Grol et al. ${ }^{17}$ This stepwise approach consists of the following actions: 1) analysis of the setting in order to identify targets for change; 2) identification of barriers and facilitators to change; 3) the development of an implementation strategy based on the in step two found barriers and facilitators; and 4) evaluation of progress. In this thesis, the stepwise approach of Grol et al. ${ }^{17}$ will be applied in order to evaluate and improve current practice of CS in The Netherlands and care for pregnant women with a prior CS in particular.

\section{Part II Improving current practice on pregnancy following caesarean section}

In The Netherlands, approximately $10 \%$ of all pregnant women have had a prior CS. ${ }^{18}$ Medical guidance of these pregnancies requires thoughtful medical attention, especially with regard to antenatal counselling on mode of delivery. Based on the results of the in part one of this thesis described analysis of current care, the second part of this thesis will elaborate on the systematically development of a care improvement strategy on counselling on mode of birth after prior CS, directed at physicians and pregnant women. The second part of the current chapter will provide an introduction to current care on birth after CS and to guideline-recommendations on counselling on pregnancy following CS in particular. 


\section{Definitions}

Several terms are used in international literature to describe mode of delivery after caesarean. In general, the term 'trial of labour after caesarean' (TOL or TOLAC) is used to describe women who try to have a vaginal birth after a prior CS. A successful TOL results in a vaginal birth after caesarean (VBAC), an unsuccessful TOL results in an (emergency) CS. In this project we use the term 'intended VBAC' instead of TOL, as this merely highlights the high probability of success instead of possible failure. When women opt for a scheduled or planned CS, this is described as an elective repeat CS (ERCS).

\section{Current care on mode of birth after $C S$}

According to contemporary guidelines on pregnancy after CS, pregnant women with a prior CS should be provided with a choice between an intended VBAC and an ERCS. ${ }^{19-22}$ Yet, worldwide, the amount of ERCSs rises ${ }^{23}$ and several hospitals in the USA even abandoned the option of intended VBAC. ${ }^{24}$ For the USA, the most recent literature shows a VBAC rate of only $8.3 \%{ }^{25}$ while earlier studies reported a rate of $50 \%$ in $1996 .{ }^{26}$ Most European countries report higher VBAC rates. For instance, current known VBAC rates in England and Wales are approximately 30-37\%.27,28 In comparison to other countries, The Netherlands have a relatively high VBAC rate: in 2002-2003 the total VBAC rate was $54 . \%^{18}$, current numbers are unknown. Supposed causes for the decreasing VBAC rates centre around the safety issue regarding the possible occurrence of uterine rupture during labour. Hence, fear for claims and litigation, insurance policies play a role ${ }^{24}$ but also the attitudes of providers and patients towards intended VBAC are changing. ${ }^{23}$

\section{Contents of antenatal counselling}

Absolute contraindications for an intended VBAC are prior uterine rupture, a vertical CS scar, three or more prior CSs, placenta previa or other factors which preclude a vaginal birth, and the inability to perform an emergency CS. ${ }^{19,29}$ The majority of women has none of these contraindications for an intended VBAC and in those cases both birth options should be offered and discussed. ${ }^{19,30}$ Within the decision making process, many factors count and therefore optimal counselling is of key importance. The following text elaborates on main factors that are regarded to be key-elements within decision-making.

\section{Probability of successful intended VBAC in relation to morbidity}

The probability that an intended VBAC results in a VBAC is generally $60-80 \% .^{23}$ Most studies which discuss risks of feto-maternal morbidity and mortality focus on the comparison of intended VBAC with ERCS. However, the probability of morbidity within 
intended VBAC also coincides with the probability that an intended VBAC results in a VBAC. ${ }^{31-33}$ This is illustrated by observations which show that the incidence of maternal morbidity is lowest in women who have a VBAC $(0.2 \%)$ in comparison to emergency CS $(3.8 \%)$ and ERCS $(0.8 \%) .{ }^{31}$ Since the incidence of morbidity within a planned CS is significantly lower than within an emergency CS, an ERCS might be the better choice for women with a low probability of successful intended VBAC. From a statistical point of view this would be the ideal approach, assuming that women could be accurately classified as 'low risk' and 'high risk' for failed intended VBAC. Several prediction models which aim to provide this calculation have been published and many provide reasonable predictability. ${ }^{34}$ However none of these models are prospectively evaluated or implemented within current care. Therefore, it is currently unknown to what extend such a prediction model would be valid and useful in clinical practice. Furthermore, current published models have been developed in diverse types of populations and settings. Before a model would be applied in Dutch practice, it should be adapted to the Dutch population.

\section{Uterine rupture}

Along general risks and benefits of a CS and vaginal delivery as described in 'part I' of this chapter, there is the probability of the occurrence of a uterine rupture during labour. A uterine rupture is the most feared complication during labour after CS, though the probability that it occurs is relatively small. However, when a uterine rupture occurs, the consequences can be severe. The probability of fetal death in case of uterine rupture is 6.2$12.4 \% .23,35$ No studies report on maternal mortality as a result of uterine rupture, however the probability of hysterectomy amongst this group is $14-33 \% .{ }^{26}$ Many cohorts with several types of settings, sample sizes and case-mix have described the incidence of uterine rupture. A recently published large case-control study revealed an incidence of $0.2 \%$ in women who had an intended VBAC. ${ }^{35}$ The earlier published meta-analysis of Guise et al. ${ }^{23}$ showed a pooled incidence of $0.47 \% .{ }^{23}$ The incidence increases when labour has been induced. There exists a large heterogeneity amongst published studies in this context, hence the quality of pooled-data is limited. A rough estimation provided by the previously named meta-analysis of Guise et al. ${ }^{23}$ shows an incidence of uterine rupture of $1.1 \%$ in women who are induced with oxytocin, $2.0 \%$ when induction occurs with prostaglandin E2 and $6.0 \%$ when induction occurs with misoprostol. ${ }^{23}$ Induction of labour using a Foley catheter seems not to effect the probability of rupture though data are limited. ${ }^{23}$ Other factors that are associated with to an increased risk of uterine rupture are two or more prior CSs, a pregnancy within twelve months of the prior CS, a prior vertical incision, macrosomia and high body mass index (BMI). ${ }^{23}$ Prior vaginal delivery seems to be protective. ${ }^{23}$, 36 Within the group of women who chooses ERCS, the mean incidence of uterine rupture is $0.03 \% .23,35$ To date, for the prediction of uterine rupture, there is no valid model available. Several 
reports show that sonographic measurements of the thickness of the CS-scar might be an powerful predictor, though there is currently no consensus on cut-off values that significantly show increase of the risk of uterine rupture ${ }^{37}$, also the methods of measurement has yet to be standardized .

\section{Fetal complications}

The total chance of fetal morbidity and mortality plays a significant role within the decisionmaking process. Apart from general risks and benefits of a CS and vaginal delivery, the total chance of fetal mortality is higher in intended VBAC, which is mainly attributable to the occurrence of uterine rupture. For intended VBAC, the weighted incidence of perinatal mortality is about $0.13 \%$, which is higher than the reported $0.05 \%$ for ERCS. ${ }^{26}$ However, to put these numbers into context, for all pregnant women the combined chance of perinatal mortality at term is approximately $0.23 \% .^{38}$ Furthermore, the probability of hypoxicischaemic encephalopathy might be higher in an intended VBAC although lack of highquality studies impair clearness about this topic. ${ }^{39,40}$

\section{Future pregnancies}

Mode of delivery in the current pregnancy has consequences for subsequent pregnancies. Studies show that with each extra CS, there is an increase of the incidence of placenta accreta, hysterectomy, IC admission, massive blood loss, operative injury and increase of duration of operation time. ${ }^{23,41}$ In absolute risks, the study of Silver et al. ${ }^{41}$ showed for placenta abnormalities an incidence of $0.31 \%$ in women having their second CS and of $0.57 \%$ for the third CS. For hysterectomy, these numbers were $0.42 \%$ and $0.90 \%$, respectively. ${ }^{1}$ Furthermore, an increasing number of studies reports on an increased risk of unexplained antepartum stillbirth in women with a uterine scar. A recent meta-analysis on this topic performed by O'Neill et al. ${ }^{42}$ suggests an increased relative risk of $23 \%$ on stillbirth for women with a prior CS compared to women without a prior CS. The aetiology however remains unravelled and confounding by indication might explain the larger part of the found association.

\section{Shared decision making}

Qualitative studies show that women experience the decision on mode of birth after CS to be a difficult decision yet they wish to be involved in decision making. ${ }^{50,51}$ Key items in the decision making process were the opinions of the providers, women's own prior experiences and expectations, experienced safety and whether information was tailored to their personal situation. ${ }^{50,52}$ Because of the complex nature of this decision-making process, 
mode of birth after CS might be discussed within a shared decision making setting. Shared decision making (SDM) is defined as a process in which the provider and patient come to a shared decision regarding a health issue. SDM is mainly practiced within preferencesensitive decisions of major importance ${ }^{53}$ and results in higher patient satisfaction, higher quality of decisions and better patient-provider communication. ${ }^{53,54} \mathrm{~A}$ patient decision aid (PtDA) is an example of a tool that can be used to effectively enhance SDM. ${ }^{55}$ In this thesis we will develop a PtDA for decision-making on mode of birth after CS in order to enhance guideline-adherence and to improve care.

\section{Aim and outline of this thesis}

In order to gain more insight into current quality of care on the practice of CSs in The Netherlands, the caesarean Section IMPLEmentation (SIMPLE) study is designed. The aim of this study is to evaluate both current physicians' adherence to guideline-based quality indicators as cognitions of medical professionals and patients on decision-making about CSs. Based on the results of the evaluation of current care, a tailored guideline implementation strategy will be developed in order to improve care.

\section{Part I Current practice of caesarean sections}

Chapter 2 defines the study protocol of the SIMPLE study. Chapter 3 describes both the development process of the guideline-based quality indicators for measurement of current Dutch practice of CSs and the results of the actual adherence to these indicators. Finally this chapter uncovers counselling of women on mode of birth after prior CS to be one of the main target points for intervention. Chapter 4 elaborates on practice variation in mode of birth after CS between Dutch hospitals.

\section{Part II Improving current practice on pregnancy following caesarean section}

Clinical prediction models and patient decision aids may help physicians in the management of patients. This concept is discussed in part II of this thesis and elaborated into the development and applicability of a guideline-implementation strategy with regard to the counselling-process of mode of birth after prior CS. Chapter 5 describes the external validation of two existing prediction models for the Dutch population, chapter 6 presents a new prediction model and chapter 7 elaborates on whether a prediction model is useful in terms of comparison to current care. Chapter 8 describes the development and pilot-testing of the developed guideline-implementation strategy. 
Chapter 9, the general discussion, highlights the implications of this thesis for current practice and future research.

\section{References}

1. Appropriate technology for birth. Lancet 1985; 2(8452): 436-7.

2. Gibbons L, Belizan JM, Lauer JA, Betran AP, Merialdi M, Althabe F. Inequities in the use of cesarean section deliveries in the world. Am J Obstet Gynecol. 2012; 206(4): 331 e1-19.

3. Kramer K, Bremer. Once a cesarean, always a cesarean? Ned Tijdscbr Geneeskd 2009; 149(B49).

4. Stichting Perinatale Registratie Nederland. Perinatale Zorg in Nederland 2013. Utrecht: Stichting Perinatale Registratie Nederland.

5. Declercq E, Young R, Cabral H, Ecker J. Is a rising cesarean delivery rate inevitable? Trends in industrialized countries, 1987 to 2007. Birth 2011; 38(2): 99-104.

6. Villar J, Carroli G, Zavaleta N, et al. Maternal and neonatal individual risks and benefits associated with caesarean delivery: multicentre prospective study. BMJ 2007; 335(7628): 1025.

7. Souza JP, Gulmezoglu A, Lumbiganon P, et al. Caesarean section without medical indications is associated with an increased risk of adverse short-term maternal outcomes: the 2004-2008 WHO Global Survey on Maternal and Perinatal Health. BMC Med 2010; 8: 71.

8. Luz Gibbons JMB, Jeremy A Lauer, Ana P Betrán, Mario Merialdi and Fernando Althabe. The Global Numbers and Costs of Additionally Needed and Unnecessary Caesarean Sections Performed per Year: Overuse as a Barrier to Universal Coverage. World Health Report (2010); Background Paper, 30.

9. National Institute for Health and Clinical Excellence. Clinical Guideline 132: Caesarean Section. London: RCOG, 2011.

10. ACOG. Safe prevention of the primary cesarean delivery. Obstetric Care Consensus No. 1. American College of Obstetricians and Gynecologists. Obstet Gynecol 2014;123:693-711..

11. NVOG - richtlijn Indicatiestelling sectio caesarea. 2011 (www.nvog.nl).

12. Boyle A, Reddy UM, Landy HJ, Huang CC, Driggers RW, Laughon SK. Primary cesarean delivery in the United States. Obstet Gynecol 2013; 122(1): 33-40.

13. Chaillet N, Dumont A. Evidence-based strategies for reducing cesarean section rates: a meta-analysis. Birth 2007; 34(1): 53-64.

14. Grol R, Grimshaw J. From best evidence to best practice: effective implementation of change in patients' care. Lancet 2003; 362(9391): 1225-30.

15. Grol R. Improving the quality of medical care: building bridges among professional pride, payer profit, and patient satisfaction. JAMA 2001; 286(20): 2578-85.

16. Chaillet N, Dube E, Dugas M, et al. Evidence-based strategies for implementing guidelines in obstetrics: a systematic review. Obstet Gynecol 2006; 108(5): 1234-45.

17. Grol R, Grimshaw J. Evidence-based implementation of evidence-based medicine. It Comm I Qual Improv. 1999; 25(10): 503-13.

18. Kwee A, Bots ML, Visser GH, Bruinse HW. Obstetric management and outcome of pregnancy in women with a history of caesarean section in The Netherlands. Eur J Obstet Gynecol Reprod Biol. 2007; 132(2): 171-6.

19. NVOG - richtlijn Zwangerschap en bevalling na een voorgaande section caesarea. 2010 (www.nvog.nl).

20. RCOG. Birth after previous caesarean birth. Green-top Guideline. 2007;No. 45.

21. SOGC. Guidelines for Vaginal Birth After Previous Caesarean Birth. J Obstet Gynaecol Can. 2005(155):89: 319-31.

22. ACOG. Vaginal birth after previous cesarean delivery. ACOG practice bulletin. 2010(no. 115).

23. Guise JM, Eden K, Emeis C, et al. Vaginal birth after cesarean: new insights. Evid Rep Technol Assess (Full Rep). 2010; (191): 1-397.

24. Roberts RG, Deutchman M, King VJ, Fryer GE, Miyoshi TJ. Changing policies on vaginal birth after cesarean: impact on access. Birth 2007; 34(4): 316-22.

25. MacDorman M, Declercq E, Menacker F. Recent trends and patterns in cesarean and vaginal birth after cesarean (VBAC) deliveries in the United States. Clin Perinatol 2011; 38(2): 179-92. 
26. National Institutes of $\mathrm{H}$. National Institutes of Health Consensus Development Conference Statement vaginal birth after cesarean: new insights March 8-10, 2010. Semin Perinatol 2010; 34(5): 351-65.

27. Royal College of Obstetricians and Gynaecologists: Birth after previous Caesarian birth iG-TGNL, UK, RCOG, 2007.

28. Montgomery AA, Emmett CL, Fahey T, et al. Two decision aids for mode of delivery among women with previous caesarean section: randomised controlled trial. BMJ 2007; 334(7607): 1305.

29. Scott J. Intrapartum management of trial of labour after caesarean delivery: evidence and experience. BJOG 2014; 121(2): 157-62.

30. Bujold E. Evaluating professional society guidelines on vaginal birth after cesarean. Semin Perinatol 2010; 34(5): 314-7.

31. McMahon MJ, Luther ER, Bowes WA, Jr., Olshan AF. Comparison of a trial of labor with an elective second cesarean section. N EnglJ Med 1996; 335(10): 689-95.

32. Cahill AG, Stamilio DM, Odibo AO, et al. Is vaginal birth after cesarean (VBAC) or elective repeat cesarean safer in women with a prior vaginal delivery? Am J Obstet Gynecol 2006; 195(4): 1143-7.

33. Grobman WA, Lai Y, Landon MB, et al. Can a prediction model for vaginal birth after cesarean also predict the probability of morbidity related to a trial of labor? Am J Obstet Gynecol 2009; 200(1): 56 e1-6.

34. Eden KB, McDonagh M, Denman MA, et al. New insights on vaginal birth after cesarean: can it be predicted? Obstet Gynecol 2010; 116(4): 967-81.

35. Fitzpatrick KE, Kurinczuk JJ, Alfirevic Z, Spark P, Brocklehurst P, Knight M. Uterine rupture by intended mode of delivery in the UK: a national case-control study. PLoS Med 2012; 9(3): e1001184.

36. de Lau H, Gremmels H, Schuitemaker NW, Kwee A. Risk of uterine rupture in women undergoing trial of labour with a history of both a caesarean section and a vaginal delivery. Arch Gynecol Obstet 2011; 284(5): 1053-8.

37. Jastrow N, Chaillet N, Roberge S, Morency AM, Lacasse Y, Bujold E. Sonographic lower uterine segment thickness and risk of uterine scar defect: a systematic review. J Obstet Gynaecol Can 2010; 32(4): 321-7.

38. Rapport a terme sterfte 2010. Perinatale audit.

39. O'Shea TM, Klebanoff MA, Signore C. Delivery after previous cesarean: long-term outcomes in the child. Semin Perinatol 2010; 34(4): 281-92.

40. Patel RM, Jain L. Delivery after previous cesarean: short-term perinatal outcomes. Semin Perinatol 2010; 34(4): 272-80.

41. Silver RM, Landon MB, Rouse DJ, et al. Maternal morbidity associated with multiple repeat cesarean deliveries. Obstet Gynecol 2006; 107(6): 1226-32.

42. O'Neill SM, Kearney PM, Kenny LC, et al. Caesarean delivery and subsequent stillbirth or miscarriage: systematic review and meta-analysis. PloS One 2013; 8(1): e54588.

43. Tita AT, Landon MB, Spong CY, et al. Timing of elective repeat cesarean delivery at term and neonatal outcomes. N Engl J Med 2009; 360(2): 111-20.

44. Grobman WA, Lai Y, Landon MB, et al. Does information available at admission for delivery improve prediction of vaginal birth after cesarean? Am J Perinatol 2009; 26(10): 693-701.

45. Scott JR. Vaginal birth after cesarean delivery: a common-sense approach. Obstet Gynecol 2011; 118(2 Pt 1): 342-50.

46. Zwart JJ, Richters JM, Ory F, de Vries JI, Bloemenkamp KW, van Roosmalen J. Uterine rupture in The Netherlands: a nationwide population-based cohort study. BJOG 2009; 116(8): 1069-78; discussion 78-80.

47. Al-Zirqi I, Stray-Pedersen B, Forsen L, Vangen S. Uterine rupture after previous caesarean section. BJOG 2010; 117(7): 809-20.

48. Rowbottom SJ, Critchley LA, Gin T. Uterine rupture and epidural analgesia during trial of labour. Anaesthesia 1997; 52(5): 486-8.

49. Holmgren C, Scott JR, Porter TF, Esplin MS, Bardsley T. Uterine rupture with attempted vaginal birth after cesarean delivery: decision-to-delivery time and neonatal outcome. Obstet Gynecol 2012; 119(4): 725-31.

50. Moffat MA, Bell JS, Porter MA, et al. Decision making about mode of delivery among pregnant women who have previously had a caesarean section: A qualitative study. BJOG 2007; 114(1): 86-93.

51. Emmett CL, Shaw AR, Montgomery AA, Murphy DJ, Di Asg. Women's experience of decision making about mode of delivery after a previous caesarean section: the role of health professionals and information about health risks. BJOG 2006; 113(12): 1438-45. 
52. Kaimal AJ, Kuppermann M. Understanding risk, patient and provider preferences, and obstetrical decision making: approach to delivery after cesarean. Semin Perinatol 2010; 34(5): 331-6.

53. van Staveren R. [Shared decision-making in medical practice--patient-centred communication skills]. Ned Tijdschr Geneeskd 2011; 155(42): A3777.

54. Stacey D, Bennett CL, Barry MJ, et al. Decision aids for people facing health treatment or screening decisions. Cochrane Database Syst Rev 2011; (10): CD001431.

55. O'Connor AM, Bennett CL, Stacey D, et al. Decision aids for people facing health treatment or screening decisions. Cocbrane Database Syst Rev 2009; (3): CD001431. 


\section{Part I}

Current practice of caesarean sections in

The Netherlands 


\section{Chapter 2}

\section{SIMPLE: Implementation of recommendations}

from international evidence-based guidelines on caesarean sections in the Netherlands. Protocol for a controlled before and after study

S Melman, ENC Schoorel, C Dirksen, A Kwee, L Smits, F de Boer, M Jonkers, MD Woiski, BWJ Mol, JPR Doornbos, H Visser, AJM Huisjes, MM Porath, FMC Delemarre, SMI Kuppens, R Aardenburg, IMA Van Dooren, FPJM Vrouenraets, FTH Lim, G Kleiverda, PCM van der Salm, K de Boer, MJ Sikkema, JG Nijhuis, RPMG Hermens,

HCJ Scheepers 


\section{Abstract}

\section{Background}

Caesarean section (CS) rates are rising worldwide. In the Netherlands, the most significant rise is observed in healthy women with a singleton in vertex position between 37 and 42 weeks gestation, whereas it is doubtful whether an improved outcome for the mother or her child was obtained. It can be hypothesized that evidence-based guidelines on CS are not implemented sufficiently.

Therefore, the present study has the following objectives: to develop quality indicators on the decision to perform a CS based on key recommendations from national and international guidelines; to use the quality indicators in order to gain insight into actual adherence of Dutch gynaecologists to guideline recommendations on the performance of a CS; to explore barriers and facilitators that have a direct effect on guideline application regarding CS; and to develop, execute, and evaluate a strategy in order to reduce the CS incidence for a similar neonatal outcome (based on the information gathered in the second and third objectives).

\section{Methods}

An independent expert panel of Dutch gynaecologists and midwives will develop a set of quality indicators on the decision to perform a CS. These indicators will be used to measure current care in 20 hospitals with a population of 1,000 women who delivered by CS, and a random selection of 1,000 women who delivered vaginally in the same period. Furthermore, by interviewing healthcare professionals and patients, the barriers and facilitators that may influence the decision to perform a CS will be measured. Based on the results, a tailor-made implementation strategy will be developed and tested in a controlled before-and-after study in 12 hospitals (six intervention, six control hospitals) with regard to effectiveness, experiences, and costs.

\section{Discussion}

This study will offer insight into the current CS care and into the hindering and facilitating factors influencing obstetrical policy on CS. Furthermore, it will allow definition of patient categories or situations in which a tailor-made implementation strategy will most likely be meaningful and cost effective, without negatively affecting the outcome for mother and child. 


\section{Background}

The worldwide rise in caesarean section (CS) rate is a major healthcare issue, with rates reported as high as 32\% in the United States (US) and 37\% in Brazil. ${ }^{1,2}$ In the Netherlands, the overall CS rate has increased from $8.1 \%$ to $13.6 \%$ over the recent decade. Although this rise is relatively low compared to other countries, a striking detail is that the most impressive rise, in absolute numbers, was among healthy women with a singleton in vertex position between 37 and 42 weeks gestation. ${ }^{3}$ However, an increasing CS rate does not imply an improved outcome for mother and infant. ${ }^{4} \mathrm{CS}$ are associated with an increased risk of maternal mortality as well as serious morbidity, such as admission to the intensive care unit (Odds Ratios (ORs) between 30.8 and 63.4), hysterectomy (ORs between 3.2 and 13.5), and puerperal infection (OR 3.0). . $^{5-7}$

Besides the short-term risks, CS have an impact on the mother's future reproductive health, for example uterine rupture, placenta praevia, or placenta accrete.,, 9

There is no evidence suggesting a better neonatal outcome from the increased CS rate in terms of mortality, intracranial haemorrhage, or impaired neurological development in the general population. ${ }^{10,11}$ In fact, an elective CS performed before 39 completed weeks is associated with respiratory distress and admission to the neonatal intensive care unit. ${ }^{11,12}$ The question arises what causes the worldwide increase in CS rate considering the fact that in most situations there are no apparent benefits of a CS for mother and child; the costs are higher compared to vaginal birth ${ }^{5}$; and the incidence of both maternal and neonatal complications are increased. There are concerns about the increasing rate of planned CS as well as a declining rate of vaginal birth after a previous CS (VBAC) in the US and Australia. ${ }^{13,14}$

To optimize CS practice, the Royal College of Obstetricians and Gynaecologists (RCOG) developed an evidence-based guideline (NICE: National Institute of Clinical Evidence) with clear recommendations for obstetric care. Similar recommendations, which have a direct effect on the decision to perform a CS, are also mentioned in the different guidelines of the Dutch Society of Obstetrics and Gynaecology (NVOG), Society of Obstetricians and Gynaecologists of Canada (SOGC), American College of Obstetrics and Gynaecology (ACOG), and National Guideline Clearinghouse from the US department of health and human services (NGC).

Despite the introduction of evidence-based guidelines, the CS rate continues to increase. We hypothesize that poor adherence to the guidelines plays a key role in the rising CS rate. In order to optimize adherence to the CS guidelines, the stepwise model by Grol can be used to select the proper strategies. ${ }^{15,16}$ The first step in this model is to analyze the current care (measured by valid quality indicators) compared to the optimal care as described in 
evidence-based guidelines, and to determine which barriers and facilitators might influence the implementation of optimal care. Subsequently, a tailor-made implementation strategy can be developed with activities applied to the determined barriers. In the last step, the strategy is executed and evaluated in terms of effectiveness, feasibility, and costs.

In view of the rising CS rate, this study aims are:

1. To develop a set of quality indicators on the decision to perform a CS based on key recommendations of both Dutch and international guidelines.

2. To gain insight into actual adherence of Dutch gynaecologists to guideline recommendations on the performance of CS.

3. To explore barriers and facilitators that have a direct effect on application of guideline recommendations regarding CS.

4. To develop, execute and evaluate a strategy in order to improve care and possibly decrease the CS incidence for a similar neonatal outcome, based on the information gathered in steps two and three.

\section{Methods}

The four aims were approached in four parts: the development of quality indicators, the assessment of current care, the identification of barriers and facilitators, and the development of a tailored implementation strategy and executing and evaluating this strategy in a clustered controlled before-and-after study (CBA).

\section{The development of quality indicators}

\section{Design and methods}

In order to measure current Dutch practice on CS, quality indicators regarding the process, structure, and outcome of care need to be developed. This will be achieved according to the RAND-modified Delphi method. ${ }^{17,} 18$ The indicators will be based on key recommendations extracted from the guidelines of several international obstetric organisations (RCOG, NVOG, SOGC, ACOG and NGC). These key recommendations will be evaluated in two rounds by an independent expert panel consisting of Dutch obstetricians and midwives. In the first round, a questionnaire will be developed on three subjects (planned CS, emergency $\mathrm{CS}$, and methods to reduce the CS rate). The questionnaire will be sent to the experts who will be asked to individually rate the key recommendations on a 9-point Likert scale ranging from 1 to 9 ('not relevant' to 'extremely relevant' for measuring the quality of CS care). 
Furthermore, a ranking of the key recommendations will be asked per subject to ultimately extract those indicators considered to be most important for quality-of-care measurement. The experts also have the opportunity to add comments or suggest additional recommendations they consider suitable as a quality indicator. The returned questionnaires will be analysed based on the ratings of the recommendations on the 9-point Likert scale, and the median score of these items will be calculated and rated as described previously by Campbell. ${ }^{19}$ Furthermore, scoring variables reflecting the ranking of the items in each of the three subjects will be developed (e.g., in a top 3 ranking, a first ranking creates 3 points, a second ranking 2 points, and a third ranking 1 point).

The second round consists of a consensus meeting where the experts will receive their individual as well as the overall results of the first round to promote discussion. The aim of this meeting is to reach consensus on those recommendations that are most suitable for assessing the quality of care on performing CS. After consensus is reached, the recommendations will be operationalized into a set of measurable quality indicators.

\section{Study population and setting}

A representative, national expert panel consisting of obstetricians and midwives (about 12 to 15 experts) will be invited. The obstetricians and midwives will have worked at various types of hospitals, ranging from small regional hospitals to university hospitals.

\section{Outcome measures}

The outcome of the first step of the study is a set of valid quality indicators regarding the decision to perform a CS the can then be used to measure the current practice.

\section{The assessment of current care}

\section{Design and methods}

A retrospective medical record search based on the set of quality indicators will be performed in order to assess the Dutch gynaecologists' adherence to the CS guideline recommendations. Adherence to these indicators will be quantified, as well as the variation in care and adherence between the participating hospitals. To gain insight into the current Dutch care compared to international care, the CS percentages of the different risk groups will be calculated according to the Robson classification (Table 2.1). ${ }^{20}$ 
Table 2.1 Ten-group classification according to Robson.

\begin{tabular}{ll}
\hline Groups & \\
\hline 1. & Nulliparous, single cephalic, $\geq 37$ weeks, in spontaneous labour \\
2. & Nulliparous, single cephalic, $\geq 37$ weeks, induced or CS before labour \\
3. & Multiparous (excluding previous CS), single cephalic, $\geq 37$ weeks, in spontaneous labour \\
4. & Multiparous (excluding previous CS), single cephalic, $\geq 37$ weeks, induced or CS before labour \\
5. & Previous CS, single cephalic, $\geq 37$ weeks \\
6. & All nulliparous breeches \\
7. & All multiparous breeches (including previous CS) \\
8. & All multiple pregnancies (including previous CS) \\
9. & All abnormal lies (including previous CS) \\
10. & All single cephalic, $\leq 36$ weeks, (including previous CS) \\
\hline
\end{tabular}

Furthermore, the maternal mortality or severe acute morbidity (Table 2.2) ${ }^{21}$, and perinatal mortality or serious morbidity ( $\mathrm{pH}<7.00$, Apgar $5 \mathrm{~min}<7$, and NICU admission) will be noted. This study will provide us with information about current practice on CS in the Netherlands and insight into the effects on outcome of mother and child.

Table 2.2 Inclusion criteria for severe acute maternal morbidity.

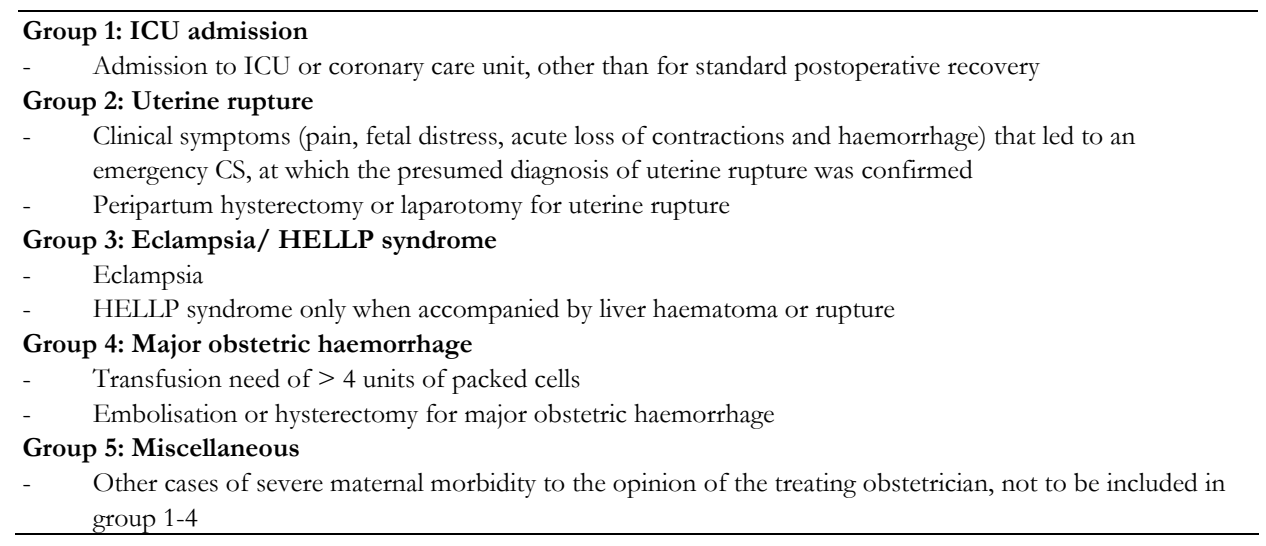

\section{Study population and setting}

In order to create a representative view of the current obstetrical care in the Netherlands, a multi-centre study will be carried out. Twenty hospitals of different Dutch regions will participate in this study, including university teaching hospitals, non-university teaching hospitals and non-university, non-teaching hospitals. The present study will take place in the setting of a Dutch Obstetric Research Consortium in which all the participating hospitals collaborate.

In the participating hospitals, data on basic obstetrical care and adherence to the quality indicators will be collected. Per hospital 100 women will be selected from the local database: 
50 women who delivered by CS and a random set of 50 women who delivered vaginally in the same time period. Exclusion criteria will be a major fetal congenital malformality and fetal death prior to onset of delivery.

\section{Outcome measures}

The main outcome is adherence to the guideline recommendations, based on the adherence to the quality indicators. Therefore, basic obstetrical data and indicator specific data will be gathered. For example, consider the indicator 'every woman with a child in breech presentation at 34 to 36 weeks gestation should be offered external cephalic version unless a contraindication for external cephalic version is present.' This implies that we need to assess the incidence of breech presentation at 34 to 36 weeks gestation, as well as data that show whether an external cephalic version is being offered. Furthermore, we will note in which cases this procedure was not offered for a valid reason. This will allow us to determine the frequencies of adherence for this indicator.

The secondary outcomes are the number of preventable CS, and Dutch practice as compared to international data using the Robson criteria.

\section{Sample size considerations}

Assuming an adherence to the guidelines of $75 \%$, an alpha of 0.05 , and a precision of the estimation of $5 \%, 300$ patients must be included. However, this number has to be adapted to take clustering of data across clinicians and within obstetrical departments into account. Assuming an intra-cluster correlation (ICC) of 0.2 and 80 professionals in 20 hospitals, 960 medical records need to be analysed. In order to compensate for loss to follow-up or incomplete data, 1,000 women with a CS will be included in 20 hospitals within a timescale of three to four months. In order to enable the calculation of specific events, as described in 'outcome measures,' a random selection of 1,000 women with a vaginal birth will be included. Thus, there will be 2,000 participants, i.e., 1,000 women after a caesarean delivery and 1,000 women after a vaginal birth. Sampling fraction will be adjusted to the fraction of women with CS in each individual hospital.

\section{Data analysis}

The frequencies of adherence per quality indicator will be calculated. This will be calculated by dividing the total number of women who apply for an indicator and for whom care was appropriate by the total number of women who apply for an indicator. For example, women with a child in breech presentation between 34 to 36 weeks of gestation, without contraindication for external cephalic version, should have been offered an external cephalic version. Adherence is the total number of women with a child in breech presentation 
between 34 to 36 weeks of gestation without contraindication for external cephalic version, in whom an external version was offered, divided by the total number of women with a child in breech presentation between 34 to 36 weeks of gestation without contraindication for external cephalic version.

\section{Barrier and facilitator study}

\section{Design and methods}

To determine the barriers and facilitators that influence the decision to perform a CS for healthcare professionals and patients, a qualitative study will be performed. The setting for guideline implementation will be analysed. Focus group interviews will be held among healthcare professionals (obstetricians, residents, and midwives) to discover factors that determine the decision to perform a CS or not. The interviewer will explore the following categories of influencing factors: features of the guidelines itself; features of the target group of professionals who should use the guidelines; features of patients who have to accept or contribute to the use of the guidelines; features of the social setting and social network of the professionals; and features of the organizational, economic, and administrative context. Remarks by professionals will be classified into categories of potential determining factors following this theoretical framework. The 'prevalence' of the features mentioned in the focus group interviews will be quantified in a survey with questionnaires among the different professionals.

Similarly, depending on the outcome of the current care study, semi-structured interviews will be held with patients in a detailed study to discover relevant factors that influence the patients' decision to choose a CS or vaginal delivery.

\section{Study population and setting}

In different hospital types (university, non-university teaching, and non-university nonteaching hospitals) interviews will be held among healthcare professionals (obstetricians, residents and midwives) as well as patients. Focus group interviews among 8 to 12 healthcare professionals will be planned. To assess whether the factors mentioned in the focus group interviews are structural, the 'prevalence' of these factors will be assessed using a survey with questionnaires among obstetric gynaecologists, residents, and midwives in the Netherlands. The questionnaires will be sent to the professionals via email addresses we will obtain from the national professional organisations of both professions. Among patients, semi-structured interviews will be held. These patients will be selected in the abovementioned hospitals from the current care study. The interviews will be conducted 
with those women belonging to the non-adherence subgroups (such as breech, nonprogressing labour, or maternal request) to whom a possible implementation should be directed. Approximately ten to fifteen women will be interviewed until no new information emerges during the interviews.

\section{Outcome measures}

The main outcome measures are the barriers and facilitators for adherence to the quality indicators for performing a CS.

\section{Data analysis}

Using Atlas, the qualitative software package, a qualitative analysis will be performed on the barriers and facilitators that are presented in the interviews among healthcare professionals and patients. The transcribed interview will be marked and coded with barriers and facilitators according to the framework used to structure the interviews: features of the guidelines, professionals, patients, social setting, and organization. These influencing factors will be quantified among all Dutch gynaecologists and midwives by means of questionnaires. The analyses of the questionnaires will be descriptive (e.g., frequencies and means).

\section{Controlled before-and-after study}

\section{Design and methods}

Based on the results of the current care study and the barrier and facilitator study, one or more target groups for a tailor-made implementation strategy will be identified. Target groups will be selected with focus on women with both a high incidence of the indicator (our hypothesis is that this will include, for example, non-progressing labour and previous CS) and low indicator adherence. A tailor-made implementation strategy will be developed in order to increase adherence to the CS quality indicators. This strategy will be executed and evaluated in a clustered CBA study in 12 hospitals (six intervention, six control hospitals) (see sample size calculation) in terms of effectiveness, experiences, and costs. It is likely that a strategy with different implementation elements is needed because several barriers for implementation of recommendations may exist at different levels. This will probably result in a combined intervention directed at the level of professionals, patients, and the organisation. 


\section{Study population and setting}

The implementation strategy will be executed and evaluated in 12 hospitals (see sample size calculation) that also participated in the current care study: six intervention hospitals in which the newly developed strategy will be applied; and six control hospitals in which care as usual will be offered. In order to select these 12 hospitals, all 20 hospitals of the current care study will be categorized into university, non-university teaching, and non-university non-teaching hospitals (three categories). Within these three categories, possible hospital pairs will be made based on pre-intervention adherence to quality indicators and CS rates, as measured in the current care study. To get a sample representative for the Dutch setting, in total two university hospitals, six non-university teaching hospitals and four non-university, non-teaching hospitals will be asked to participate. Subsequently, the participating hospitals have to be assigned to the intervention and control group. This will be done per stratum and based on geographic region. The evaluation will include an effect, process, and cost analysis. Just as in de current care study, the effects will be measured both at medical outcome level (i.e., CS rates and complication rates) and on guideline adherence level. Satisfaction with and applicability of the tailor-made implementation strategy for both patients and healthcare professionals will be measured in a process evaluation. Information regarding the process will be gathered in a qualitative study in the hospitals in which the implementation strategy was applied. Individual interviews will take place among the involved healthcare professionals and patients to gather data about experiences with the changed care. During the interview, they also will be asked about which elements of the tested strategy they specifically used to implement the evidence-based guidelines; how satisfied they are with the different elements; and their opinion about the feasibility of the different elements.

Furthermore, a cost analyses of the tested implementation strategy will take place with respect to three aspects: 1) the rate at which the guideline recommendations are already applied; 2) the costs of the implementation strategy (taking into account the development of the strategy, training of healthcare professionals, and possible extra costs regarding both time and medical costs) and 3) the effectiveness of the implementation strategy. In order to measure the effectiveness of the implementation strategy both the effects on medical outcome (i.e., CS rates, complication rates) and adherence to CS quality indicators will be measured. The cost-effectiveness of the implementation strategy will be expressed as the incremental costs per extra patient treated according to the CS quality indicators, compared to the 'do-nothing' strategy. 


\section{Outcome measures}

The primary outcome is effectiveness of the implementation strategy, which is defined as the observed increase in adherence to the developed quality indicators with regard to the chosen target group (for example non-progressing labour or previous CS) between the intervention and control hospitals and the actual CS rates in both groups. Secondary outcome measures are experiences and satisfaction of healthcare providers and patients with the implementation strategy as well as applicability and costs.

\section{Sample size considerations}

For a sample size calculation, the target group and the adherence to the quality indicators regarding this target group are necessary. These data will be available after performing the current care as well as the barrier study. Based on these data, the sample size can be calculated. In this calculation, we will take into account clustering of patients within professionals and hospitals. We expect most of the clustering at professional level and presume an inclusion of a number of professionals per hospital and a number of patients per professional. For pragmatic reasons, we will include at most 12 out of the 20 hospitals of the current care measurement. This sample of hospitals has to be representative for the Dutch setting, i.e., a total of two university hospitals, six non-university teaching hospitals, and four non-university, non-teaching hospitals.

\section{Data analysis}

To assess the effectiveness of the implementation strategy, the proportion of patients that are treated in accordance with the guidelines before and after implementation of the guidelines in both the intervention and control hospitals will be measured. Medical outcome measures will include CS rates and maternal as well as neonatal complications related to vaginal delivery or CS. Multilevel multivariate analysis will be carried out to assess the independent effect of the implementation strategy on adherence to the CS quality indicators and medical outcome measures. A qualitative descriptive analysis will be done in order to evaluate the process. Furthermore, by means of a questionnaire, the experiences of healthcare providers and patient satisfaction with the implementation strategy will be evaluated, and the outcomes will be descriptive.

The costs analysis will be performed from a healthcare perspective. The costs of the implementation process will be calculated on the basis of the time and materials invested based on activity-based costing (ABC) approach, focusing on activities performed with costs accumulated at the activity level(s) of the healthcare implementation processes. The costs of implementation of the guidelines and consolidation consist of personnel and material costs. The input of resources will be assessed by collecting volumes of consumed 
resources, and multiplying these by the price of each resource unit. For collecting information on the input of the resources, registration forms will be completed by the people involved in the implementation and consolidation process. The prices of each resource unit will be based on standard $\operatorname{costs}^{22}$, market prices, or self-determined costs. The medical costs used in de cost analysis will include CS rates and maternal and neonatal complications related to vaginal delivery or CS. The costs of implementation and costs of the changed medical care will be weighed against the proportion of patients that are treated according to the CS guideline, after implementation. The cost-effectiveness of implementation will be expressed as the incremental costs per extra patient treated according to the CS guideline, compared to the 'do-nothing' strategy (i.e., no implementation, for which data before implementation will be used).

\section{Ethical considerations}

The Medical Ethical Committee (CMO) of Maastricht (azM/UM) declared that no ethical approval was necessary for this study protocol.

\section{Discussion}

The CS rate in The Netherlands is comparatively low compared to other countries, but it is increasing especially in the group of healthy women with a singleton pregnancy in vertex position at term. One would expect this to coincide with improved outcomes for mothers and children, which is, however, not the case. ${ }^{5}$ Although many suggestions considering the reason for this rise have been made, the answer is not yet clear. We hypothesize that incomplete guideline adherence is a possible cause for the current increase in CS rate. In this study we will determine current Dutch care regarding CS using quality indicators. We will use national as well as international guidelines to select the recommendations, resulting in at least a set of internationally accepted indicators. Because we will also report the current CS rates by classification into the internationally accepted Robson Criteria, international comparison of incidences of CS rates in different subgroups will be possible. In that way, specific indicators and incidences of those indicators might be applicable elsewhere. Furthermore, we will focus on factors that influence guideline implementation, and thus optimal care in the barrier and facilitator analysis.

Although earlier reviews claimed that multifaceted strategies (combinations of many different interventions) are often effective, Grimshaw found that a higher number of intervention components was not related to higher effectiveness. ${ }^{23}$ It seems plausible that combined interventions are only more effective than single interventions, if these are 
addressed at the specific barriers to change. This is also the conclusion of Chaillet et al:: in the obstetric setting in general and the CS setting in particular, prospective identification of efficient strategies and barriers to change is necessary to achieve a better adaptation of intervention and to improve clinical practice guideline implementation. ${ }^{24}$

This study will hopefully result in one or more target groups with high incidence and low guideline adherence and the evaluation of an implementation strategy to improve care. For example, non-progressing labour is known to be one of the major reasons to perform a CS. Should guideline adherence in these women be low, an intervention based both on informing women and reminders on optimal care for caregivers could be an option. Another possible target group might be women with a previous CS. Although in general, the VBAC rate was previously reported higher in the Netherlands than in some other countries, recent data are lacking. Improvement of care for these women could consist of a decision aid to improve counselling. Both types of interventions are also possibly effective outside the Netherlands. The ultimate aim of our study is to implement the national and international evidence-based guidelines on CS in all Dutch hospitals in order to reduce the incidence of CS and improve the outcome for mother and child. Furthermore, this study provides a framework for future studies to enable improvement of guideline adherence and reduction of the CS rate.

\section{References}

1. Menacker F, Hamilton BE. Recent trends in cesarean delivery in the United States. NCHS data brief 2010; (35): 1-8.

2. Betran AP, Merialdi M, Lauer JA, et al. Rates of caesarean section: analysis of global, regional and national estimates. Paediatr Perinatal Epidemiol 2007; 21(2): 98-113.

3. Kwee A, Elferink-Stinkens PM, Reuwer PJ, Bruinse HW. Trends in obstetric interventions in the Dutch obstetrical care system in the period 1993-2002. Eur J Obstet Gynecol Reprod Biol 2007; 132(1): 70-5.

4. Villar J, Carroli G, Zavaleta N, et al. Maternal and neonatal individual risks and benefits associated with caesarean delivery: multicentre prospective study. BMJ 2007; 335(7628): 1025.

5. Souza JP, Gulmezoglu A, Lumbiganon P, et al. Caesarean section without medical indications is associated with an increased risk of adverse short-term maternal outcomes: the 2004-2008 WHO Global Survey on Maternal and Perinatal Health. BMC Med 2010; 8: 71.

6. Liu S, Liston RM, Joseph KS, et al. Maternal mortality and severe morbidity associated with low-risk planned cesarean delivery versus planned vaginal delivery at term. CMAJ 2007; 176(4): 455-60.

7. Lee YM, D'Alton ME. Cesarean delivery on maternal request: maternal and neonatal complications. Curr Opin Obstet Gynecol 2008; 20(6): 597-601.

8. Ananth CV, Smulian JC, Vintzileos AM. The association of placenta previa with history of cesarean delivery and abortion: a metaanalysis. Am J Obstet Gynecol 1997; 177(5): 1071-8.

9. Rossi AC, Lee RH, Chmait RH. Emergency postpartum hysterectomy for uncontrolled postpartum bleeding: a systematic review. Obstet Gynecol 2010; 115(3): 637-44.

10. Tita AT, Landon MB, Spong CY, et al. Timing of elective repeat cesarean delivery at term and neonatal outcomes. N Engl J Med 2009; 360(2): 111-20. 
11. Yee W, Amin H, Wood S. Elective cesarean delivery, neonatal intensive care unit admission, and neonatal respiratory distress. Obstet Gynecol 2008; 111(4): 823-8.

12. Wilmink FA, Hukkelhoven CW, Lunshof S, Mol BW, van der Post JA, Papatsonis DN. Neonatal outcome following elective cesarean section beyond 37 weeks of gestation: a 7-year retrospective analysis of a national registry. Am J Obstet Gynecol 2010; 202(3): 250 e1-8.

13. MacDorman M, Declercq E, Menacker F. Recent trends and patterns in cesarean and vaginal birth after cesarean (VBAC) deliveries in the United States. Clin Perinatol 2011; 38(2): 179-92.

14. Zhang J, Troendle J, Reddy UM, et al. Contemporary cesarean delivery practice in the United States. $A m J$ Obstet Gynecol 2010; 203(4): 326 e1- e10.

15. Grol R. Personal paper. Beliefs and evidence in changing clinical practice. BMJ 1997; 315(7105): 418-21.

16. Grol R. Improving the quality of medical care: building bridges among professional pride, payer profit, and patient satisfaction. JAMA 2001; 286(20): 2578-85.

17. Hermens RP, Ouwens MM, Vonk-Okhuijsen SY, et al. Development of quality indicators for diagnosis and treatment of patients with non-small cell lung cancer: a first step toward implementing a multidisciplinary, evidence-based guideline. Lung Cancer 2006; 54(1): 117-24.

18. Mourad SM, Hermens RP, Nelen WL, Braat DD, Grol RP, Kremer JA. Guideline-based development of quality indicators for subfertility care. Hum Reprod 2007; 22(10): 2665-72.

19. Campbell SM, Braspenning J, Hutchinson A, Marshall M. Research methods used in developing and applying quality indicators in primary care. Qual Saf Health Care 2002; 11(4): 358-64.

20. Robson MS. Can we reduce the caesarean section rate? Best Pract Res Clin Obstet Gynaecol.2001; 15(1): 179-94.

21. van Dillen J, Mesman JA, Zwart JJ, Bloemenkamp KW, van Roosmalen J. Introducing maternal morbidity audit in the Netherlands. BJOG 2010; 117(4): 416-21.

22. Oostenbrink JB, Buijs-Van der Woude T, van Agthoven M, Koopmanschap MA, Rutten FF. Unit costs of inpatient hospital days. Pharmacoeconomics 2003; 21(4): 263-71.

23. Grimshaw JM, Thomas RE, MacLennan G, et al. Effectiveness and efficiency of guideline dissemination and implementation strategies. Health Technol Assess 2004; 8(6): iii-iv, 1-72.

24. Chaillet N, Dumont A. Evidence-based strategies for reducing cesarean section rates: a meta-analysis. Birth 2007; 34(1): 53-64. 


\section{Chapter 3}

\section{Development and measurement of guidelines-based}

quality indicators of caesarean section care in the Netherlands: a RAND-modified Delphi procedure and retrospective medical chart review

S Melman, ENC Schoorel, K de Boer, H Burggraaf, JB Derks, D van Dijk, J van Dillen, CD Dirksen, JJ Duvekot, A Franx, THM Hasaart, AJM Huisjes, D Kolkman, S. van Kuijk, A Kwee, BW Mol, MG van Pampus, A de Roon-Immerzeel, JJM van Roosmalen, FJME Roumen, E Smid-Koopman, L Smits, WA Spaans, H Visser, WJ van Wijngaarden, C Willekes, MGAJ Wouters, JG Nijhuis, RPMG Hermens, HCJ Scheepers 


\section{Abstract}

\section{Objective}

Development and measurement of quality indicators to determine guideline adherence and target groups for improvement on care with direct effect on caesarean section (CS) rates.

\section{Design}

RAND-modified Delphi method and a retrospective medical chart review.

\section{Setting}

Multi-centre study, including twenty-one hospitals.

\section{Population}

Eighteen obstetricians and midwives participated in an expert panel for indicator development. Charts of 1024 women with a CS and a stratified and weighted randomly selected group of 1036 women with a vaginal delivery (VD) were analysed.

\section{Method}

A RAND-modified Delphi method was used to systematically develop quality indicators, with focus on counselling and preventive measures in CS care. Quality indicators frequency and adherence were scored in 2060 women with CS or VD.

\section{Main outcome measures}

Indicator adherence, defined as the number of women in a specific obstetrical situation in which care was performed as recommended in both planned and emergency CS care.

\section{Results}

The expert panel developed 16 indicators on planned CS and 11 indicators on emergency CS. The most frequently occurring situations with low indicator adherence were: 1) suspected fetal distress (frequency 17\%, adherence 46\%), 2) non-progressive labour (frequency $12 \%$, CS performed too early in over 75\%), 3) continuous support during labour (frequency $88 \%$, adherence $37 \%$ ) and 4) previous CS (frequency $12 \%$ ), with adequate counselling in $15 \%$.

\section{Conclusions}

Based on 27 developed quality indicators, we identified four target groups for improvement of care. 


\section{Background}

There is a worldwide rise in caesarean section (CS) rates. Although the Netherlands has a relatively low CS rate $(16.7 \%)$ compared to the United Kingdom $(24.6 \%)$, the most impressive rise in CS rate is found in 'low risk pregnancies': healthy women with a singleton in vertex position at term. ${ }^{1-4}$ Furthermore, rising CS rates are not associated with improved outcome for mother and neonate. Although a CS is a relatively safe procedure, it is associated with increased short term morbidity and mortality, with an increased risk of abnormal placentation and uterine rupture in future pregnancies. ${ }^{5-10}$ The World Health Organization estimates a CS rate between $10-15 \%$ to be optimal. ${ }^{11}$. A CS costs twice as much as a vaginal delivery, (1256 to 9652 euro extra depending on the country of origin (Daily Mail July 2013)). Adding all costs of future morbidity and increased risk of future repeat CS, the estimated additional costs of one CS are 7500 euro.

The cause of the increasing CS rate is still unknown. Previous studies mostly focus on epidemiological data such as rising maternal age, maternal request for CS and decline in attempt of vaginal birth after CS. ${ }^{12,13}$ Our hypothesis is, that incomplete adherence to guidelines regarding the decision when to perform a CS might be an important explanation for the rising CS rate. This hypothesis is supported by hospital-level variation in CS rates, which cannot be explained by socio-demographic or clinical factors. ${ }^{12}$

In recent decades, several international obstetrical organizations have developed evidencebased guidelines with recommendations for optimal care regarding the decision when to perform a CS. However, the crucial issue remains whether these recommendations are actually followed. In order to improve current CS care, it is of importance to gain insight into the extent of guideline implementation in daily practice. Before this can be measured, valid quality indicators for optimal care have to be systematically developed. ${ }^{14,15}$

In the present study, we apply a systematic method for development of evidence-based quality indicators. Based on these indicators, we compare current Dutch care to optimal care as described in international evidence-based guidelines. This will allow the identification of target groups of women in which a tailor-made implementation strategy might improve care and reduce CS rates.

\section{Methods}

\section{Development of CS quality indicators}

A systematic RAND-modified Delphi method was used to select a set of key recommendations appropriate for transcription into quality indicators. ${ }^{15,16}$ These recommendations were extracted from national guidelines (NVOG: Dutch Society of 
Obstetricians and Gynaecologists, CBO: Centraal Begeleidings Orgaan, a Dutch organization aiming at improving the quality of care by health care professionals), international guidelines (RCOG: Royal College of Obstetricians and Gynaecologists, ACOG: American Congress of Obstetricians and Gynaecologists, and SOGC: Society of Obstetricians and Gynaecologists of Canada) and literature. ${ }^{15}$ The national expert panel consisted of both obstetricians $(\mathrm{N}=13)$ and midwives $(\mathrm{N}=4)$ and were members of either the Dutch Society of Obstetrics and Gynaecology (NVOG) or the Royal Dutch Organization of Midwives (KNOV). The experts rated and discussed indicators on planned CS (including mode of delivery counselling and CS prevention) as well as indicators on emergency CS in an iterative way. The exact procedure for indicator development is described in Appendix S1 (Stepwise procedure of CS quality indicators).

\section{Measurement of CS current care}

\section{Design and setting}

We conducted a retrospective multi-centre cohort study. This study was situated within the Dutch Obstetric Consortium, which is a research collaboration of obstetric clinics in The Netherlands (http://www.studies-obsgyn.nl).

\section{Study population}

In order to obtain a representative view of current CS care, this study was conducted in 21 hospitals: 5 university hospitals, 10 non-university teaching hospitals and 6 non-university, non-teaching hospitals located in different regions of The Netherlands. To gain real insight in current obstetrical care and measure quality indicator adherence, women with a CS and women with a vaginal delivery (VD) were included. For example, consider the situation of breech presentation. In such a situation, an external cephalic version should be offered. In order to study guideline adherence, all women with a fetus in breech presentation after 34 weeks need to be identified. In this case, adequate care is offering external cephalic version to women with a breech presentation after 34 weeks, independent of their acceptance or the result of the attempt.

Per hospital, the medical charts of 50 consecutive women who underwent a caesarean delivery as well as a random selection of 50 women who underwent a VD in the same period, were analysed. Since it was inefficient to include and analyse all women with a VD, a randomization list was developed per hospital based on the local CS rate. For example, if the CS rate was $20 \%$, VD sampling rate was $0.25(0.25 * 80 \%=20 \%)$. We excluded cases with major fetal abnormalities (defined as 'abnormalities that interfere with standard obstetrical care or vaginal birth'), birth prior to 24 weeks of gestation and fetal demise prior to onset of delivery. 


\section{Data collection}

The data were gathered by trained research nurses from the Dutch Obstetric Consortium (http://www.studies-obsgyn.nl). We extracted basic obstetrical data for all women from their individual medical charts; including data on previous deliveries (previous VD, CS) and current pregnancy (parity, singleton/ multiple gestation). Furthermore, we gathered indicator specific data for all women to enable calculation of adherence to each indicator. These data included conditions that might influence mode of delivery and existed prior to delivery (diabetes, hypertension) or developed either during pregnancy (suspected fetal macrosomia, intrauterine growth restriction) or during delivery (suspected fetal distress, non-progressive labour). Indicator specific data included ultrasound results, mode of delivery counselling, delivery specifics (eg. use of ST-analysis or fetal scalp blood sampling, pain medication and oxytocin). We assessed indicator specific data for all women in order to evaluate whether care was provided according to guidelines.

\section{Sample size}

We assumed a mean adherence to the guidelines of $75 \%$, an alpha of 0.05 , and a precision of the estimation of $5 \%$. Next, we assumed an intra-cluster correlation (ICC) of 0.2 and 80 professionals in 20 hospitals. Taking clustering of data across clinicians and within obstetrical departments into account, 960 medical charts were needed for analyses. In order to compensate for loss to follow-up or incomplete data, at least 1,000 women with a CS needed to be included.

In order to enable the calculation of specific events (frequencies) as described in 'measurement of CS quality indicators', a random selection of 1000 women with a vaginal delivery were included. This resulted in the analysis of 50 women with a VD and 50 women with a CS per hospital.

\section{Statistical analysis}

To assess guideline adherence, performance scores per indicator were calculated, ranging from 0 to $100 \%$. This was done as follows: the number of women to whom the indicator applied and actual care was consistent with the indicator (numerator) was divided by the total number of women to whom the indicator applied (denominator). When an indicator was composed of aggregated items (e.g. the indicator 'request for CS without medical grounds'), we calculated additional sub percentages for each item. In this case, sub percentages were calculated for 3 additional items: 1. explore reason for CS request, 2. discuss (dis)advantages to CS delivery and 3. Offer psychological counselling in case of fear of delivery. Analyses were performed using SPSS statistics 21.

Percentages were weighted for the hospital-specific sampling fractions used in sampling VDs. For example, if a sampling fraction of 0.25 was used in a particular hospital, data for 
each VD from that hospital counted four (1/0.25) times in the calculation of numerators and denominators.

\section{Results}

\section{Development of CS quality indicators}

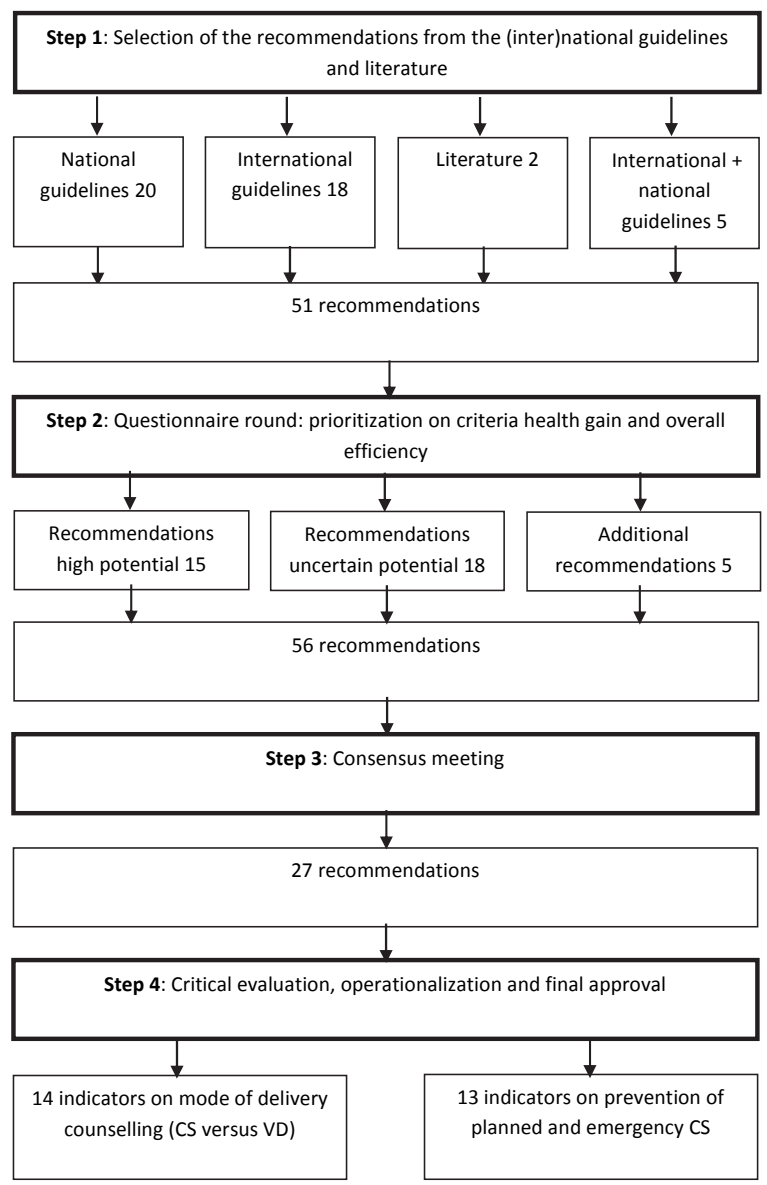

Figure 3.1 Development of quality indicators.

Based on 51 recommendations, extracted from the guidelines, the stepwise procedure of indicator development (figure 3.1) resulted in a set of 27 CS quality indicators, including 16 indicators on planned CS (mode of delivery counselling (CS versus VD) and prevention) as 
well as 11 indicators on emergency CS. The final indicator set is given in Table 3.1. The indicators on planned CS are shown in Table S3.1 (Quality indicators: planned CS is advised) in the appendix of this paper.

Table 3.1 Quality indicator set.

1) Quality indicators on planned CS

a) General counselling, CS is not mentioned (VD is the normal conduct)

1. Twin pregnancy and first child cephalic position

2. Fetal macrosomia $(<4.5 \mathrm{~kg}$ in maternal diabetes, $<5 \mathrm{~kg}$ no maternal diabetes)

3. Preterm labour, cephalic position

4. Small for gestational age without fetal distress

5. Previous shoulder dystocia without impaired perinatal outcome

b) Counselling directed at VD (VD and CS are options, VD is preferred)

6. Position of the placenta at $1-2 \mathrm{~cm}$ of the internal os Request for CS without medical grounds:

7. Explore reason for request

8. Discuss (dis)advantages to CS delivery

9. In case of extreme fear: offer psychological counselling

10. Preterm breech delivery (frank, complete breech)

c) Counselling mentioning both VD and CS as equal options

11. Breech presentation at term

Previous CS (Inform on risks and chance for successful VBAC)

12. Inform on low risk of uterine rupture

13. Inform on high chance of successful VBAC

14. Inform on increased risk and lower success rate in case of need for labour induction

d) Prevention of planned CS

15. Offer external cephalic version in case of non-cephalic position

16. Use of internal audit on CS

2) Quality indicators on emergency CS

17. In case of suspected fetal distress use STAN (ST analysis) or micro blood analysis

In case of non-progressive labour first stage:

18. Rupture of membranes,

19. Urinary catheterization,

20. Use of pain medication, preferably epidural analgesia,

21. Adequate contractions or augmentation of labour

In case of non-progressive labour second stage in nulliparous women:

22. Active pushing recommended,

23. Adequate contractions recommended,

24. Consider vacuum extraction if the head is $<1 / 5^{\text {th }}$ palpable per

Abdomen

25. Continuous support during labour for women with or without prior training

26. Use of partogram

27. Involvement of consultant obstetrician in decision making for CS

\section{Study population}

All 21 hospitals were asked to provide data of $50 \mathrm{VD}$ as well as $50 \mathrm{CS}$, which would result in a study population of 2100 women. There were 22 women who met the exclusion criteria. Not every hospital analysed the requested 100 women, resulting in an analysis of 
2060 women. When adjusting for the random selection of vaginal deliveries, these 2060 women represent a total study population of 4687 women.

\section{Measurement of current care}

Table 3.2 shows the frequency of specific obstetrical events, as described by the indicators, as well as the performance scores (indicating adequate care) in the total study population ( $N=4687$ women) concerning: 1. planned CS and 2 emergency CS.

Table 3.2 Weighted frequencies of CS quality indicators.

\begin{tabular}{|c|c|c|}
\hline Quality indicators on planned CS & $\begin{array}{l}\text { Frequency of } \\
\text { occurrence }\end{array}$ & $\begin{array}{c}\text { Perfomance } \\
\text { score } \\
\text { (adherence) }\end{array}$ \\
\hline \multicolumn{3}{|l|}{ General counselling, $C S$ is not mentioned (VD is the normal conduct) } \\
\hline Twin pregnancy and first child in cephalic position & $1.3 \%$ & $16 \%$ \\
\hline Fetal macrosomia ( $<4.5 \mathrm{~kg}$ in maternal diabetes, $<5 \mathrm{~kg}$ no maternal diabetes) & $4.3 \%$ & $33 \%$ \\
\hline Preterm labor and cephalic position & $4.7 \%$ & $45 \%$ \\
\hline Small for gestational age without fetal distress & $3.3 \%$ & $43 \%$ \\
\hline Previous shoulder dystocia no impaired outcome child & $1.1 \%$ & $22 \%$ \\
\hline \multicolumn{3}{|l|}{ Counselling directed at VD (VD and CS are options, VD is preferred) } \\
\hline Position of the placenta at $1-2 \mathrm{~cm}$ of the internal os & $0.02 \%$ & $100 \%$ \\
\hline Request for CS without medical grounds: & $1.0 \%$ & \\
\hline Discussion on reason request & & $80 \%$ \\
\hline Discussion on (dis)advantages to CS & & $66 \%$ \\
\hline Offer psychological counselling & & $62 \%$ \\
\hline Preterm breech delivery (frank, complete breech) & $1.7 \%$ & $1.3 \%$ \\
\hline \multicolumn{3}{|l|}{ Counselling mentioning both VD and CS as equal options } \\
\hline Breech position at term & $4.1 \%$ & $56 \%$ \\
\hline Previous CS (inform on risks and success rates) & $11.7 \%$ & $4 \%$ \\
\hline $\begin{array}{l}\text { Previous CS and medical reason for induction of labor (inform on risks and success } \\
\text { rates) }\end{array}$ & $2.2 \%$ & $18 \%$ \\
\hline \multicolumn{3}{|l|}{ Prevention of planned CS } \\
\hline Offer external cephalic version for non-cephalic position & $6 \%$ & $77 \%$ \\
\hline \multicolumn{3}{|l|}{ Quality indicators on emergency CS: } \\
\hline Use of partogram & $6.9 \%$ & $54 \%$ \\
\hline Use of STAN (ST analysis) or micro blood analysis in case of suspected fetal distress & $16.9 \%$ & $46 \%$ \\
\hline In case of non-progressive labor, perform or provide: & $11.1 \%$ & \\
\hline A AROM (artificial rupture of membranes) & & $95 \%$ \\
\hline B Urinary catheterization & & $61 \%$ \\
\hline C Use of pain medication & & $78 \%$ \\
\hline D Preferably use of epidural analgesia & & $49 \%$ \\
\hline E Adequate contractions & & $93 \%$ \\
\hline Before performing a CS, an optimal situation (A-E) $>2 \mathrm{hrs}$ & & $23 \%$ \\
\hline Before performing a CS, an optimal situation (A-E) $>4$ hrs & & $15 \%$ \\
\hline $\begin{array}{l}\text { In case of a primipara and non-progressive labor second stage the following needs to } \\
\text { be done: }\end{array}$ & $12.7 \%$ & \\
\hline Active pushing & & $98 \%$ \\
\hline Adequate contractions & & $72 \%$ \\
\hline \multicolumn{3}{|l|}{ Vacuum extraction considered if the head is } \\
\hline$<1 / 5^{\text {th }}$ palpable per abdomen & & $45 \%$ \\
\hline Continuous support during labor & $88.3 \%$ & $37 \%$ \\
\hline
\end{tabular}




\section{Planned caesarean section}

Table 3.2 shows that for planned CS, the frequency of the occurrence of the specific events ranged from $0.02 \%$ to $11.7 \%$ and adequate care (performance scores) ranged from 4 to $100 \%$. Although in many obstetric situations caregivers do not follow guidelines, the impact on total caesarean section rate is not likely to change since the frequency of the situation is very low. This is the case for twin pregnancies with the first fetus in cephalic position, preterm breech and previous shoulder dystocia, occurring in less than $2 \%$ in the general population. The population with a high incidence and a low performance are women with a previous CS. In an average obstetric population, $11.7 \%$ of all women have a previous CS and in only $15 \%$ counselling regarding estimated success rates of a VD, next to risks and benefits involved with CS and VD according to the guidelines was documented. In addition, in only $4 \%$ of the medical charts of these women, comments informing on risks and benefits were detailed. The highest performance scores for this group of women were found for mode of delivery counselling in case of placenta position at 1-2 cm of the internal os $(100 \%)$, to offer external cephalic version for non-cephalic position $(77 \%)$ and counselling on CS without medical grounds (62-80\%).

\section{Emergency caesarean section}

Unlike the indicators for planned caesarean sections, the indicators for emergency caesarean sections have a much higher frequency of occurrence, ranging from 11 to $88.3 \%$. In these indicators, guideline adherence in general is higher ranging from 23 to $98 \%$.

Continuous support during labour was advised for all women starting vaginal birth. In $37 \%$ of these women the support was actually provided. It was advised to apply additional diagnostics such as ST-analysis or fetal scalp blood sampling to all women with suspected fetal distress, if this was technically possible and no contraindications existed to the procedure. In $46 \%$ of the women with suspected fetal distress, additional diagnostics were applied before proceeding to a CS. In women with non-progressive labour, the performance scores of the separate quality indicators (artificial rupture of membranes, urinary catheterization, use of pain medication (preferably epidural analgesia and adequate contractions), ranged from $61 \%$ to $95 \%$. However, the expert panel advised to proceed to a CS based on non-progression, not earlier than 2- 4 hours after all previous measures were fulfilled. Only in a small proportion of women, these criteria were met and in more than $77 \%$ CS were performed to soon. 


\section{Discussion}

\section{Main findings}

This study resulted in a set of 27 evidence-based quality indicators on both planned as emergency CS. Current care measurement in the Netherlands identified four major target groups for future implementation strategies due to their high prevalence and low adherence rate: improvement of counselling in women with a previous CS, improvement of implementation of continuous support during labour, additional diagnostics before proceeding to a CS in case of suspected fetal distress and allowing a longer waiting period before proceeding to a CS in case of non-progressive labour.

\section{Strengths and limitations}

Our study offers the first set of CS indicators covering entire obstetrical care, thereby enabling measurement of quality of obstetrical care in situations that exist antepartum (e.g. breech presentation), intrapartum (e.g. non-progressive labour) as well as postpartum (e.g. internal audit on CS). This is in contrast to previously developed indicators which only focussed on peripartum care. ${ }^{17}$

By including 21 hospitals, including different types of hospitals in several regions, and analysing more than 1000 women per group, our results are a good representation of actual care in The Netherlands.

Although a standardised method for the development of quality indicators was used, there are several limitations to this study. To date no study compared the different methods used for quality indicator development. However, the RAND-modified Delphi method offers a systematic approach to indicator development and is a frequently used method that has proven to result in valid quality indicators. ${ }^{18}$

Another limitation is that the expert panel consisted of Dutch healthcare providers. One can challenge whether all quality indicators are usable and accepted in different and specific obstetrical settings. However, the obstetrical situations described (e.g. macrosomia, previous CS) are comparable world-wide and the basis of the quality indicators consists of recommendations derived from international guidelines and literature. Although there may be a different approach in obstetrical care in some cases (e.g. preterm breech delivery), we expect a similar approach by most obstetrical healthcare professionals to the identified major categories. Therefore, we believe that the most important quality indicators are likely to be adopted by most obstetrical healthcare professionals. 
The data collection from medical charts was performed by trained research nurses from the Dutch consortium, which could introduce bias. It was shown by Luck et al. ${ }^{19}$ that medical chart review somewhat underestimates the actual care given. Not every detail of a consultation is noted in the medical chart. Secondly, despite the fact that the data were extracted by trained research employees, there might be interpretation bias. However, when considering the adherence percentages for the main categories (fetal distress, nonprogressive labour and previous CS) in our study, we do not expect our results to change substantially.

\section{Interpretation}

In case of a rare situation like triplets, discussing the necessity of CS will only lead to marginal improvement of general care. However, an improvement strategy will have a considerably larger effect in case of a situation with a relatively high frequency and low nonadherence rate, such as non-progressive labour. The CS quality indicators allowed us to analyse obstetrical care in the Netherlands, thereby identifying groups of women in whom a high frequency of a certain quality indicator is observed in combination with low adherence. The next step in improvement of care will be to determine factors that influence the mode of delivery decision, by either facilitating or hindering quality indicator adherence. Based on these influencing factors, a tailor-made implementation strategy is expected to have a high impact on obstetrical care.

When the Dutch results are compared to international data, it is expected that for most indications for performing a CS, incidences are not very different. Therefore, interventions that are directed to these situations are likely to have a high impact internationally. Nonprogressive labour is globally a very common problem and a high CS rate is observed. Intervention in this group is likely to have a high impact on current care. In women with suspected fetal distress, the incidence of the problem is likely to be comparable as well, but management in the United States can probably be improved with the introduction of STanalysis or fetal blood sampling.

Our findings on adherence in the (preventive measures of) emergency CS (continuous support during labour, suspected fetal distress and non-progressive labour) are consistent with the idea that it is important to prevent the first caesarean delivery. A low adherence rate in these cases could, in part, explain the rising CS rate for healthy women with a singleton in vertex position at term. ${ }^{20-23}$

In general, the cost of a CS is about double that of a vaginal delivery. After a first CS, a large majority of women have a CS in the subsequent pregnancy, ranging from 18 to $72 \%$ depending on the country women live in. ${ }^{24}$ For each repeated CS, the morbidity increases, with higher risks of operative complications, blood transfusions, IC admittance and 
hysterectomy, adding extra costs. Therefore, with on average one subsequent pregnancy, the extra costs are estimated to approach 7500 Euro. In Europe, 5.2 million women delivered in 2012, with on average a 30\% CS rate. With a reduction of 1\%, in Europe alone 390 million Euro can be saved. The WHO advocates a CS rate between 10-15\%, although this has been challenged. This would result in a cost reduction of 3.9 billion euro every year, with no likely harmful effect on maternal or neonatal outcomes.

Beside the use of the presented indicators for local improvement, a subset could be used for international comparison of CS care. Until now, international comparison was directed at classification and numbers of CS, but this does not reflect quality of care. ${ }^{25-27}$ We believe that international comparison of CS care could be directed at the subset of indicators that have the highest impact: 1) in women with a previous CS, structured information on risks and benefits on vaginal delivery compared to planned CS should be given and women should be given a choice; 2) women should be offered continuous support during vaginal delivery 3) before performing CS for suspected distress, fetal blood sampling or ST- analysis should be performed; 4) before performing CS for non-progressive labour, a 2-4 hour waiting period should be installed after a situation with ruptured membranes, adequate contractions and adequate pain relief is established.

\section{Conclusion}

This study provides a framework for future studies for improvement of guideline adherence and reduction of CS rates, thereby possibly improving the outcome for mother and child. Due to the relatively high frequency of occurrence in combination with a low adherence rate, we identified 4 possible target groups of women where a tailor-made implementation strategy could improve CS care. The next step will be to identify barriers and facilitators that influence guideline adherence. We identified several quality indicators that are likely to be used for improvement of care on an international basis, after measurement of local current care as well as local facilitating and hindering factors.

\section{References}

1. Stichting Perinatale Registratie Nederland. Perinatale Zorg in Nederland 2012. Utrecht: Stichting Perinatale Registratie Nederland.

2. Kwee A, Bots ML, Visser GH, Bruinse HW. Obstetric management and outcome of pregnancy in women with a history of caesarean section in the Netherlands. Eur J Obstet Gynecol Reprod Biol 2007; 132(2): 171-6.

3. Information Centre. NHS maternity statistics E-DoH, 2009. 
4. Menacker F, Hamilton BE. Recent trends in cesarean delivery in the United States. NCHS data brief 2010; (35): 1-8.

5. Liu S, Liston RM, Joseph KS, et al. Maternal mortality and severe morbidity associated with low-risk planned cesarean delivery versus planned vaginal delivery at term. CMAJ 2007; 176(4): 455-60.

6. Lumbiganon P, Laopaiboon M, Gulmezoglu AM, et al. Method of delivery and pregnancy outcomes in Asia: the WHO global survey on maternal and perinatal health 2007-08. Lancet 2010; 375(9713): 490-9.

7. Ananth CV, Smulian JC, Vintzileos AM. The association of placenta previa with history of cesarean delivery and abortion: a metaanalysis. Am J Obstet Gynecol 1997; 177(5): 1071-8.

8. Rossi AC, Lee RH, Chmait RH. Emergency postpartum hysterectomy for uncontrolled postpartum bleeding: a systematic review. Obstet Gynecol 2010; 115(3): 637-44.

9. Zwart JJ, Richters JM, Ory F, de Vries JI, Bloemenkamp KW, van Roosmalen J. Uterine rupture in The Netherlands: a nationwide population-based cohort study. BJOG 2009; 116(8): 1069-78; discussion 78-80.

10. van Dillen J, Mesman JA, Zwart JJ, Bloemenkamp KW, van Roosmalen J. Introducing maternal morbidity audit in the Netherlands. BJOG 2010; 117(4): 416-21.

11. Appropriate technology for birth. Lancet 1985; 2(8452): 436-7.

12. Bragg F, Cromwell DA, Edozien LC, et al. Variation in rates of caesarean section among English NHS trusts after accounting for maternal and clinical risk: cross sectional study. BMJ 2010; 341: c5065.

13. Menacker F, Declercq E, Macdorman MF. Cesarean delivery: background, trends, and epidemiology. Semin Perinatol 2006; 30(5): 235-41.

14. Grol R, Baker R, Moss F. Quality improvement research: understanding the science of change in health care. Qual Saf Health Care 2002; 11(2): 110-1.

15. Campbell SM, Braspenning J, Hutchinson A, Marshall M. Research methods used in developing and applying quality indicators in primary care. Qual Saf Health Care 2002; 11(4): 358-64.

16. Silverberg MJ, Thorsen P, Lindeberg H, Grant LA, Shah KV. Condyloma in pregnancy is strongly predictive of juvenile-onset recurrent respiratory papillomatosis. Obstet Gynecol 2003; 101(4): 645-52.

17. Kesmodel US, Jolving LR. Measuring and improving quality in obstetrics--the implementation of national indicators in Denmark. Acta Obstet Gynecol Scand 2011; 90(4): 295-304.

18. Kotter T, Blozik E, Scherer M. Methods for the guideline-based development of quality indicators--a systematic review. Implement Sci 2012; 7: 21.

19. Luck J, Peabody JW, Dresselhaus TR, Lee M, Glassman P. How well does chart abstraction measure quality? A prospective comparison of standardized patients with the medical record. Am J Med 2000; 108(8): 642-9.

20. Kwee A, Elferink-Stinkens PM, Reuwer PJ, Bruinse HW. Trends in obstetric interventions in the Dutch obstetrical care system in the period 1993-2002. Eur J Obstet Gynecol Reprod Biol 2007; 132(1): 70-5.

21. American College of O, Gynecologists, Society for Maternal-Fetal M, et al. Safe prevention of the primary cesarean delivery. Am J Obstet Gynecol 2014; 210(3): 179-93.

22. Delbaere I, Cammu H, Martens E, Tency I, Martens G, Temmerman M. Limiting the caesarean section rate in low risk pregnancies is key to lowering the trend of increased abdominal deliveries: an observational study. BMC Pregnancy Childbirth 2012; 12: 3.

23. Haerskjold A, Hegaard HK, Kjaergaard H. Emergency caesarean section in low risk nulliparous women. $J$ Obstet Gynaecol 2012; 32(6): 543-7.

24. Guise JM, Eden K, Emeis C, et al. Vaginal birth after cesarean: new insights. Evid Rep Technol Assess (Full Rep). 2010; (191): 1-397.

25. Chong C, Su LL, Biswas A. Changing trends of cesarean section births by the Robson Ten Group Classification in a tertiary teaching hospital. Acta Obstet Gynecol Scand 2012; 91(12): 1422-7.

26. Colais P, Fantini MP, Fusco D, et al. Risk adjustment models for interhospital comparison of CS rates using Robson's ten group classification system and other socio-demographic and clinical variables. BMC Pregnancy Childbirth 2012; 12: 54.

27. Farine D, Shepherd D. Classification of caesarean sections in Canada: the Modified Robson criteria. JOGC 2012; 34(10): 976-83. 


\section{Appendix S1. Stepwise procedure of CS quality indicators}

\section{Methods}

The method of indicator development was carried out according to four consecutive steps: 1) identification of recommendations, 2) questionnaire round, 3) consensus meeting and 4) critical evaluation and operationalization.

\section{Step 1: Identification of recommendations}

Five international guidelines were used to extract recommendations: guidelines of the ACOG $(n=1)$, RCOG $(n=3)$ and SOGC $(n=1)$. In addition, all national guidelines of the Dutch Society of Obstetricians and Gynaecologists (NVOG) with recommendations that may affect CS rates were used (e.g. fetal surveillance during labour, breech presentation, twin pregnancy etc.). Furthermore, the guideline on antiretroviral therapy by the CBO (Centraal BegeleidingsOrgaan), a Dutch organization aiming at improving the quality of care by health care professionals, was selected. In addition, the articles of Silverberg et al. and Robson et al. were used for their information on condyloma treatment in pregnancy and medical audit to reduce CS rates, respectively. ${ }^{14,15}$

Subsequently, the recommendations were categorised as follows: 1) planned CS (including recommendations on counselling on vaginal delivery (VD) versus caesarean section (CS) in different situations and prevention of planned CS) 2) emergency CS. The recommendations considering counselling include situations in which a) general counselling is advised without mentioning the possibility of choosing CS (VD is the normal conduct); b) counselling is advised directed at VD (VD and CS are both options but VD is preferable; c) counselling is advised mentioning both VD and CS as equal options; and d) planned CS is advised.

\section{Step 2: Questionnaire round}

The identified recommendations were subdivided according to the abovementioned categories and were transformed into a questionnaire that was sent to the expert panel. If available, the questionnaire contained the evidence level per recommendation in order to support the decision-making process. The experts were asked to rate each recommendation on a nine-point Likert scale with respect to their value for both health gain and overall efficacy. In order to be able to distinguish between recommendations with a high score on the Likert scale, a ranking of recommendations per category was used. Furthermore, the experts were requested to add complementary recommendations. This part of the procedure resulted in an allocation of the recommendations for the consensus meeting into recommendations with high, uncertain or low potential as quality indicator.

Recommendations were considered 'high potential' if: 1) the median rating of the recommendation was 8 or more; 2 ) the recommendation was in the 
top ranking of the specific category and had at least $20 \%$ of the maximum score and 3) $70 \%$ or more of the expert panel ratings were in the highest tertile on the Likert scale (7, 8 or 9). There were three possible appraisal combinations that rated the recommendation as 'uncertain potential': 1) a median score of 8 or more and a top ranking for less than $20 \%$ of the maximum score; 2) a median score less than 8 and a ranking for at least $20 \%$ of the maximum score or 3 ) a rating of $30 \%$ or more in the highest $(7,8$ or 9$)$ and lowest tertile (1, 2 or 3 ) on the Likert scale (=disagreement). The recommendation was determined to have 'low potential' if none of the abovementioned criteria were applicable. ${ }^{16}$

\section{Step 3: Consensus meeting}

In a face-to-face consensus meeting the results of the questionnaire round were discussed. In order to improve the debate each expert was provided with the overall results from the questionnaire round, as well as their own ratings and additional remarks. Firstly, the experts were asked if they agreed that the recommendations marked 'high potential' indeed had to be selected as potential quality indicators to assess and monitor quality of care, and whether or not they could agree to the dismissal of those recommendations considered 'low potential' as quality indicators. In addition, the experts were requested if there were any recommendations marked as 'uncertain potential' they would strongly advise to consider as an indicator. The members of the expert panel were finally asked which additional recommendations proposed by an expert would be suitable as quality indicator.

\section{Step 4: Critical evaluation and operationalization}

After the consensus meeting the results were offered to all the members of the expert panel for final approval. Subsequently, the selected recommendations were translated into indicators by defining numerators and denominators, i.e. the number of women in whom a certain test or intervention should have been performed and has been performed, divided by the number of women in whom a certain test or intervention should have been performed. Using this method, an adherence percentage could be calculated in order to assess current care.

\section{Results}

\section{Step 1: Identification of recommendations}

In the first step, 51 recommendations on CS were identified: 16 situations where VD was the preferred mode of delivery (category $1 \mathrm{a}$ and $1 \mathrm{~b}$ ); 9 situations in which counselling was advised on the possibility of choosing either vaginal or caesarean delivery (category 1c); 2 recommendations on prevention of planned CS (category 1d), and 14 situations where planned CS was advised. Furthermore, there were 8 recommendations on emergency CS. 


\section{Step 2: Questionnaire round}

The 17 experts each received a questionnaire, of which 16 were returned (1 non-responder, $94 \%$ response rate). Out of the 51 recommendations, 14 had 'high potential', 18 had 'uncertain potential' and 19 had 'low potential'. The experts suggested 5 additional recommendations.

\section{Step 3: Consensus meeting}

Eight experts attended the consensus meeting (47\%), 5 obstetricians and 3 midwives. This meeting resulted in a consensus-based set of 27 key recommendations labelled according to two categories [See Table 3.1]. Category 1 comprised 14 recommendations on mode of delivery counselling: 1a) general counselling is advised without mentioning the possibility of choosing CS (VD is the normal conduct) (N=5); 1b) counselling is advised directed at VD (VD and CS are both options but VD is preferable) (N=5), 1c) counselling is advised mentioning both VD and CS as equal options $(\mathrm{N}=4)$. There were 2 recommendations on prevention of planned CS (1d). The experts approved of 11 recommendations on the prevention of emergency CS (category 2).

With regard to the situations were planned CS was advised (category 1d), the 12 indications were not considered to be of high potential for measuring quality of care. For example, in case of placenta praevia, it is not likely that an obstetrician would proceed with VD. Consequently, it is not expected that adherence to such an indicator would be low, and improvement of care would be necessary.

\section{Step 4: Critical evaluation, operationalization and final approval}

The 27 key recommendations were sent to the expert panel for final approval, which was obtained from 16 experts; there was 1 non-responder.

Finally, the selected recommendations were translated into indicators by defining numerators and denominators: i.e. the number of women in whom a certain intervention or counselling method should have been performed and has been performed, divided by the number of women in whom a certain intervention or counselling method should have been performed. For example: one of the indicators states that in case of breech presentation after 34 weeks, external cephalic version should be offered. Guideline adherence then is measured by dividing the number of women in the study group with a fetus in breech presentation after 34 weeks in whom external cephalic version is offered by the total number of women with a fetus in breech presentation after 34 weeks.

This stepwise procedure resulted in a set of 27 CS quality indicators, including 16 indicators on planned CS as well as 11 indicators on emergency CS. The process of development of quality indicators on CS by using the RAND-modified Delphi method is depicted in figure 3.1, the final indicator set is given in Table 3.1. 


\section{Appendix S2. Quality indicators: planned CS is advised}

Table S3.1 Quality indicators: planned CS is advised.

Planned CS is advised:

Suspected placental abruption and viable fetus

Vasa praevia

Placenta praevia

Placental localization less than $1 \mathrm{~cm}$ from the internal os

Relevant scar in the uterus (e.g. vertical incision during previous CS)

Monoamniotic twin pregnancy

Breech presentation at term and maternal pelvic abnormality

Breech presentation at term and previous non-progressive labor

First genital herpes outbreak in the third trimester of pregnancy

Impossible vaginal birth (e.g. due to cervical myomas, congenital malformations)

Uterine rupture in previous pregnancy

Persistent transverse presentation, despite external cephalic version 


\section{Chapter 4}

\section{Practice variation of vaginal birth after caesarean: a registration based cohort study}

ENC Schoorel, E Vankan, SM van Kuijk, BWJ Mol, JG Nijhuis, R Aardenburg, K de Boer, FMC Delemarre, CD Dirksen, IM van Dooren, MTM Franssen, M Kaplan, G Kleiverda, SMI Kuppens, A Kwee, FTH Lim, S Melman, MM Sikkema, E Smid-Koopman, LJ Smits, H Visser, J Langenveld, M Woiski, HC Scheepers, RP Hermens 


\section{Abstract}

\section{Objective}

To analyse practice variation of vaginal birth after caesarean (VBAC) and successful intended VBAC, adjusted for differences in case mix.

\section{Study design}

This nationwide registry based cohort study was performed amongst a representative group of Dutch hospitals $(n=17)$. Women with one prior caesarean section (CS) without a contraindication for intended VBAC were included. We used backwards stepwise multivariate logistic regression analysis to develop models for case mix correction. One model was derived to adjust the elective repeat caesarean section (ERCS) rates; a second model was derived to adjust successful intended VBAC rates. Standardized rates of ERCS and successful intended VBAC per hospital were established and compared. R-squared measures were obtained for estimation of the total percentage of practice variation explained by the models.

\section{Results}

We reviewed 9833 consecutive medical records, 1068 women (11\%) had a history of CS of whom $763(71 \%)$ were eligible for inclusion. Five-hundred-fifteen women $(67 \%)$ had an intended VBAC of whom $72 \%$ successfully delivered vaginally. Amongst the 17 participating hospitals, the ERCS rate ranged from $6 \%$ to $54 \%$, with a mean of 29.8 , standard deviation (SD) $11.8 \%$. The mean observed successful intended VBAC rate ranged from $52.7 \%$ to $90.0 \%$ (mean $71.8 \%$, SD $11.1 \%$ )). More than $85 \%$ of the observed practice variation could not be explained by case mix.

\section{Conclusions}

In The Netherlands intended VBAC is common practice, but the a priori probability of having a CS independent of woman's individual risk factors varies strongly per hospital. 


\section{Introduction}

In 1985, the World Health Organization stated that the ideal caesarean section (CS) rate is around $10-15 \% .{ }^{1}$ Nevertheless, the frequency of CS's has continued to rapidly increase in many parts of the world. Consequently, the number of women who are pregnant following a CS increased concordantly resulting in more women at risk for a (repeat) CS. ${ }^{2}$ The debate concerning birth after CS centres around safety in terms of the unlikely but severe event of occurrence of uterine rupture. On the other hand, an elective repeat caesarean section (ERCS) is, in comparison to an intended vaginal birth after caesarean (VBAC), associated with an increased risk of morbidity in future pregnancies and higher costs. ${ }^{2}$ Hence, for the United States (US), one of the 'Healthy People 2020' goals is to reduce the amount of ERCSs from $90.8 \%$ to $81.7 \% .^{3}$ In order to achieve this goal, clear guidelines were developed that advice on how to counsel women on mode of birth after CS and how to organize care. ${ }^{4-8}$ In this counselling, women should be given information including benefits and risks of both options and ideally, shared decision making has to take place. However, studies show large practice variation in intended VBAC rates ${ }^{2,9}$ both at national and international level, suggesting large variation in implementation of contemporary guidelines. Yet, these studies did not correct for case mix, hence these results should be interpreted with caution as variation in patient populations might induce this practice variation. In order to accurately analyse practice variation we aim to evaluate practice variation in mode of section after CS and particularly to what extent it can be explained by factors on patient level.

\section{Materials and methods}

\section{Design and setting}

In a nationwide registration based cohort study we compared care regarding mode of section for women who are pregnant after a CS, amongst 17 Dutch hospitals in terms of practice variation.

All enrolled hospitals participated in the Dutch consortium of obstetrics and gynaecology and were representative for Dutch geographic regions and hospital types. The present study was performed in academic teaching hospitals $(n=5)$, teaching non-academic hospitals $(n=7)$ and non-teaching non-academic hospitals $(n=5)$. Consecutive deliveries were recorded per site starting at January $1^{\text {st }} 2010$. All enrolled sites were requested to include 30 consecutive women who had an intended VBAC and all women who had an ERCS in the same timeinterval. This resulted in an overview of all women who had delivered within the set time- 
interval and had 0,1 or $\geq 2$ prior CS's. Subsequently, a database was constructed that included all women who had one prior CS.

\section{Study population}

We included women with a history of one prior CS and a vertex singleton pregnancy who delivered at $\geq 37$ weeks gestational age (GA). Women were excluded when they had an unknown indication of prior CS, or in the current pregnancy an intra-uterine fetal demise or a contraindication for an intended VBAC. A contraindication for intended VBAC was defined as a previous uterine rupture, a placenta previa or a relevant uterine scar.

\section{Outcome measures}

The main outcome measures of this study were the amount of variation in ERCS- and successful intended VBAC rates between hospitals corrected for variables on patient level (i.e. case mix). Secondary outcome measures were the differences in practice variation between hospital types and whether there was a correlation between standardized ERCSand intended VBAC-success rates.

\section{Data collection}

We collected data on frequencies of mode of section after CS per hospital. To correct for case mix in each case, data were gathered with regard to demographic factors (maternal age and ethnicity), past obstetric factors and pre-existing and current obstetric factors. Obstetric factors included variables that are predictive for mode of birth after CS and for successful intended VBAC. These variables were chosen based on published prediction models on successful intended VBAC, original research articles that report on predictors for mode of birth after CS, VBAC and on expert opinion. ${ }^{10,11}$ The variables considered relevant for case mix correction included maternal age, pre-pregnancy body-mass-index (BMI), ethnicity, prior non-progressive labour, any prior VBAC, any prior vaginal delivery, estimated fetal weight $\geq \mathrm{p} 90$, estimated fetal weight $\leq \mathrm{p} 10$, diabetes mellitus, hypertension, preeclampsia/ HELLP syndrome. Pre-pregnancy BMI was defined as $\mathrm{kg} / \mathrm{m}^{2}$ obtained pre-pregnancy or within the first trimester. Estimated fetal weight was measured in the third trimester by either ultrasound or upon physical examination. Diabetes mellitus and hypertension could be either pre-existent or pregnancy-induced. For prediction of successful intended VBAC we added to this set whether labour was induced and labour parameters upon presentation to the labour ward (cervical dilation, cervical effacement and fetal station). Cervical dilation was registered per centimetre (range 0-10). Cervical effacement was categorized in three measures: $0-25 \%, 25-50 \%,>50 \%$. Fetal station was recorded using the in the Netherlands customary 'Hodge classification system' (range $\mathrm{H} 0-\mathrm{H} 4$ ) and converted to the American 
classification system ranging from ballotable $(B)$ to +5 . This variable was defined as follows: $\mathrm{H} 0=-5, \mathrm{H} 1=-3, \mathrm{H} 2=-1, \mathrm{H} 3=0, \mathrm{H} 4=+3$.

Data were extracted from medical records by trained research staff using customized case report forms. Data were checked for completeness and inconsistencies. Inconsistent and incomplete data were double-checked directly with the hospital concerned.

\section{Statistical analyses}

Missing data were imputed using stochastic regression imputation since omission of incomplete cases can result in loss of precision and may bias the results. ${ }^{12,13}$ For imputation, all quantitative baseline characteristics were used for estimation of the missing values.

For case mix correction, we derived two multivariate logistic regression models that predicted ERCS and successful intended VBAC, respectively. One model was used to correct ERCS rates for case mix, while the second model was used to correct successful intended VBAC rates for case mix. The multivariate logistic regression models were developed using a backwards stepwise elimination method. At first, we performed univariate analyses to assess which case mix variables could be related to the outcome variables. In order to minimize the risk of exclusion of important variables, we used a liberal p-value of $0.2^{10}$ to estimate which variables were univariate significantly related to the outcome variables. Subsequently, the multivariate logistic regression formula was applied to the dataset to calculate both the probability of ERCS and successful intended VBAC per woman. Mean predicted outcomes per hospital represented the expected ERCS- and successful intended VBAC rates per hospital. Expected ERCS and successful intended VBAC rates per hospital were compared to the 'true', 'observed' rates. Accordingly, for each hospital, standardized rates were computed by dividing observed rates by expected rates and multiplying it by the population mean. The standardized rate represents the ERCS and intended VBAC rates that the hospital would have if all hospitals had a similar patient population. R-squared measures were obtained for estimation of the total percentage of practice variation that was explained by the models. Subsequently correlation between standardized ERCS rates and successful intended VBAC rates was tested using Spearman's rank correlation test (reference $\mathrm{p}<0.05$ ) (since the data was not normally distributed) and by visually inspecting the plot.

Statistical analyses were performed using SPSS (SPSS version 20.0). 


\section{Ethical approval}

Approval for this study was obtained at the Medical Ethical Committee (CMO) of Maastricht University Medical Centre+ (MUMC+) in The Netherlands (MEC number 09-4047-13).

\section{Results}

\section{Study population}

We reviewed 9833 consecutive medical records, and 1068 women (11\%) had a history of CS of whom $763(71 \%)$ met the inclusion criteria. The baseline characteristics of this study cohort are shown in Table 4.1.

Table 4.1 Baseline characteristics of study population.

\begin{tabular}{|c|c|c|}
\hline Characteristic $(n=763)$ & $\begin{array}{l}\text { Missing data intended VBAC; ERCS } \\
\qquad(\mathrm{n} ; \mathrm{n})\end{array}$ & $\begin{array}{l}\text { Women } \\
n=763\end{array}$ \\
\hline Maternal age (years, mean $\pm \mathrm{SD}$ ) & $2 ; 4$ & $32.5(4.5)$ \\
\hline Ethnicity $(\mathrm{n},(\%))$ & $15 ; 12$ & \\
\hline Caucasian & & $608(79.7)$ \\
\hline Mediterranean & & $48(6.3)$ \\
\hline African & & $30(3.9)$ \\
\hline Indo-Surinamese & & $8(1.0)$ \\
\hline Asian & & $19(2.5)$ \\
\hline Other & & $23(3.0)$ \\
\hline Unknown & & $27(3.5)$ \\
\hline Previous CS due to failure to progress $(\mathrm{n},(\%))$ & $0 ; 0$ & $336(44.0)$ \\
\hline Any previous vaginal delivery $(\mathrm{n},(\%))$ & $0 ; 0$ & $152(19.9)$ \\
\hline Previous VBAC $(\mathrm{n},(\%))$ & $0 ; 0$ & $107(14.0)$ \\
\hline First trimester BMI $\left(\mathrm{kg} / \mathrm{m}^{2}\right.$, mean $\left.\pm \mathrm{SD}\right)$ & $124 ; 79$ & $26.1(5.9)$ \\
\hline PE/HELLP (n, (\%)) & $0 ; 2$ & $15(2.0)$ \\
\hline Hypertension (n, (\%)) & $1 ; 3$ & $50(6.6)$ \\
\hline Diabetes mellitus ( $\mathrm{n},(\%))$ & $2 ; 1$ & $34(4.5)$ \\
\hline $\mathrm{EFW} \leq \mathrm{p} 10(\mathrm{n},(\%))$ & $201 ; 105$ & $38(5.0)$ \\
\hline $\mathrm{EFW} \geq \mathrm{p} 90(\mathrm{n},(\%))$ & $201 ; 105$ & $22(2.9)$ \\
\hline Induction of labour $(\mathrm{n},(\%))$ & $0 ; \mathrm{b}$ & $132(26) / b$ \\
\hline Cervical dilation $(\mathrm{cm}$, mean $\pm \mathrm{SD})$ & $11 ; \mathrm{b}$ & $3 \pm 2 / b$ \\
\hline Cervical effacement $(\%$, mean \pm SD $)$ & $56 ; \mathrm{b}$ & $64 \pm 19 / b$ \\
\hline Fetal station $(B,-5-+5$, mean $\pm S D)$ & $57 ; \mathrm{b}$ & $-2 \pm 2 / b$ \\
\hline
\end{tabular}

VBAC: vaginal birth after caesarean, ERCS: elective repeat caesarean section, SD: standard deviation; CS: caesarean section; BMI, body mass index; PE: pre-eclampsia; HELLP: HELLP syndrome - hemolysis, elevated liverenzymes, low platelets; EFW: estimated fetal weight B, ballottement. 
On average, maternal age, ethnicity and having preeclampsia/HELLP or hypertension or an EFW $\leq$ p10 did not differ between ERCS and intended VBAC.

In our cohort, 248 (33\%) women had an ERCS. The other 515 women (67\%) had an intended VBAC of whom $371(72 \%)$ had a successful intended VBAC, resulting in a VBAC rate of $49 \%$. Women who chose ERCS had more often a prior CS due to failure to progress, had diabetes mellitus, had a higher BMI or an EFW $\geq$ p90. Women who chose ERCS had less often a prior vaginal delivery or prior VBAC.

\section{ERCS rates corrected for case mix}

We entered all preselected variables in the regression model in order to develop a model for case mix correction. These results are shown in Table 4.2. The variables that showed significance for case mix correction of the ERCS rates were pre-pregnancy BMI, prior nonprogressive labour, prior VBAC and diabetes mellitus.

Table 4.2 Overview of multivariate logistic regression model for predicting ERCS.

\begin{tabular}{lccccc}
\hline Variable & Coefficient & SE & p-value & Odds ratio & 95\% CI \\
\hline Intercept & -3.012 & 0.897 & 0.001 & 0.049 & - \\
Maternal age (years) & 0.034 & 0.023 & 0.144 & 1.034 & $0.989-1.082$ \\
Pre-pregnancy BMI $\left(\mathrm{kg} / \mathrm{m}^{2}\right.$ ) & 0.038 & 0.016 & 0.016 & 1.039 & $1.007-1.072$ \\
Prior non-progressive labour (yes/no) & 0.578 & 0.199 & 0.004 & 1.783 & $1.207-2.634$ \\
Prior VBAC (yes/no) & -1.454 & 0.399 & 0.000 & 0.234 & $0.107-0.510$ \\
Diabetes mellitus (yes/no) & 1.391 & 0.466 & 0.003 & 4.020 & $1.613-10.018$ \\
Hypertension (yes/no) & -0.550 & 0.406 & 0.175 & 0.577 & $0.260-1.278$ \\
\hline
\end{tabular}

ERCS: elective repeat caesarean section, SE: standard error, CI: confidence interval, BMI: body mass index, VBAC: vaginal birth after caesarean.

Table 4.3 shows the number of included deliveries per hospital, the observed ERCS rates, expected ERCS rates and standardized ERCS rates. Also, the mean differences between the expected and standardized rates are shown, which represent the amount of CSs that a hospital deviates from its expected rate when it would be presented with the mean patient population. Amongst the 17 participating hospitals, the mean crude observed ERCS rate was $29.8 \% \pm 11.8 \%$ (range $6.0 \%$ to $54.0 \%$ ). After correction for case mix, $86.1 \%$ of the observed practice variation remained unexplained. 
Table 4.3 Standardized ERCS rates.

\begin{tabular}{lcccccc}
\hline $\begin{array}{l}\text { Hospital } \\
\text { type }\end{array}$ & $\begin{array}{c}\text { Hospital } \\
\text { number }\end{array}$ & $\begin{array}{c}\text { Included } \\
\text { deliveries }\end{array}$ & $\begin{array}{c}\text { Observed } \\
\text { rate (\%) }\end{array}$ & $\begin{array}{c}\text { Expected } \\
\text { rate (\%) }\end{array}$ & $\begin{array}{c}\text { Standardized } \\
\text { rate (\%) }\end{array}$ & $\begin{array}{c}\text { Mean difference } \\
\text { (\%) expected - } \\
\text { standardized }\end{array}$ \\
\hline 1 & 1 & 61 & 54.0 & 34.7 & 51.4 & -16.8 \\
1 & 2 & 51 & 39.0 & 33.3 & 38.7 & -5.5 \\
1 & 4 & 45 & 33.0 & 33.6 & 32.4 & 1.3 \\
1 & 12 & 55 & 38.0 & 34.7 & 36.1 & -1.4 \\
1 & 15 & 40 & 43.0 & 29.0 & 47.8 & -18.7 \\
2 & 5 & 28 & 32.0 & 33.9 & 31.1 & 2.8 \\
2 & 7 & 36 & 17.0 & 31.5 & 17.8 & 13.7 \\
2 & 9 & 39 & 28.0 & 30.6 & 30.2 & 0.5 \\
2 & 10 & 41 & 24.0 & 29.6 & 26.8 & 2.8 \\
2 & 11 & 48 & 35.0 & 32.9 & 35.1 & -2.2 \\
2 & 13 & 24 & 17.0 & 28.1 & 19.9 & 8.2 \\
2 & 16 & 42 & 26.0 & 31.7 & 27.1 & 5.7 \\
3 & 3 & 39 & 23.0 & 29.3 & 25.9 & 3.4 \\
3 & 6 & 100 & 41.0 & 32.4 & 41.8 & -9.4 \\
3 & 8 & 36 & 17.0 & 31.5 & 17.8 & 13.7 \\
3 & 14 & 46 & 35.0 & 31.9 & 36.2 & -4.2 \\
3 & 17 & 32 & 6.0 & 28.6 & 6.9 & 21.7 \\
\hline
\end{tabular}

Hospital types: 1: non-teaching non-academic hospital, 2: teaching non-academic hospital, 3: academic teaching hospital

\section{Successful intended VBAC rates corrected for case mix}

The from univariate analyses derived significant variables for correction of VBAC-rates were maternal age, pre-pregnancy BMI, prior non-progressive labour, prior vaginal delivery, Caucasian ethnicity, cervical dilation and cervical effacement. These data are shown in more detail in Table 4.4.

Table 4.4 Overview of multivariate logistic regression model for predicting successful intended VBAC.

\begin{tabular}{lccccc}
\hline Variable & Coefficient & SE & p-value & Odds ratio & 95\% CI \\
\hline Intercept & 1.886 & 1.259 & 0.134 & 6.591 & - \\
Maternal age (years) & -0.045 & 0.030 & 0.130 & 0.956 & $0.903-1.013$ \\
Pre-pregnancy BMI $\left(\mathrm{kg} / \mathrm{m}^{2}\right)$ & -0.047 & 0.021 & 0.025 & 0.954 & $0.916-0.994$ \\
Prior non-progressive labour (yes/no) & -0.426 & 0.259 & 0.100 & 0.653 & $0.393-1.085$ \\
Prior vaginal delivery (yes/no) & 1.612 & 0.402 & 0.000 & 5.011 & $2.278-11.026$ \\
Caucasian (yes/no) & 0.582 & 0.349 & 0.096 & 1.790 & $0.903-3.550$ \\
Cervical dilation (yes/no) & 0.104 & 0.067 & 0.122 & 1.110 & $0.973-1.267$ \\
Cervical effacement (yes/no) & 0.292 & 0.157 & 0.063 & 1.339 & $0.984-1.821$ \\
\hline
\end{tabular}

VBAC: vaginal birth after caesarean, SE: standard error, CI: confidence interval, BMI: body mass index

Table 4.5 shows observed, expected and standardized intended VBAC rates, as the amount that a hospital deviates from its expected rate when it would be presented with the mean 
patient population. Amongst the 17 included hospitals, the mean crude observed VBAC rate was $71.8 \% \pm 11.1 \%$ (range $50.0 \%$ to $90.0 \%$ ). After correction for case mix, $85.3 \%$ of the observed practice variation remained unexplained.

Table 4.5 Standardized successful intended VBAC rates.

\begin{tabular}{lcccccc}
\hline $\begin{array}{l}\text { Hospital } \\
\text { type }\end{array}$ & $\begin{array}{c}\text { Hospital } \\
\text { number }\end{array}$ & $\begin{array}{c}\text { Included } \\
\text { deliveries }\end{array}$ & $\begin{array}{c}\text { Observed } \\
\text { rate (\%) }\end{array}$ & $\begin{array}{c}\text { Expected } \\
\text { rate (\%) }\end{array}$ & $\begin{array}{c}\text { Standardized } \\
\text { rate (\%) }\end{array}$ & $\begin{array}{c}\text { Mean difference } \\
\text { (\%) expected - } \\
\text { standardized }\end{array}$ \\
\hline 1 & 1 & 28 & 60.7 & 72.1 & 60.7 & 11.4 \\
1 & 2 & 31 & 71.0 & 68.3 & 74.9 & -6.6 \\
1 & 4 & 30 & 83.3 & 74.5 & 80.7 & -6.2 \\
1 & 12 & 34 & 70.6 & 73.2 & 69.5 & 3.8 \\
1 & 15 & 23 & 50.0 & 68.5 & 54.9 & 13.6 \\
2 & 5 & 19 & 68.4 & 62.0 & 79.6 & -17.6 \\
2 & 7 & 30 & 86.7 & 77.1 & 81.1 & -4.0 \\
2 & 9 & 29 & 71.4 & 73.2 & 67.9 & 5.3 \\
2 & 10 & 31 & 86.7 & 70.1 & 89.1 & -19.0 \\
2 & 11 & 31 & 71.0 & 65.8 & 77.8 & -12.0 \\
2 & 13 & 20 & 90.0 & 71.2 & 91.2 & -19.9 \\
2 & 16 & 31 & 77.4 & 71.5 & 78.1 & -6.6 \\
3 & 3 & 30 & 70.0 & 72.3 & 69.8 & 2.5 \\
3 & 6 & 59 & 76.3 & 75.3 & 73.1 & 2.3 \\
3 & 8 & 30 & 56.7 & 73.4 & 55.7 & 17.8 \\
3 & 14 & 30 & 56.7 & 65.7 & 62.2 & 3.5 \\
3 & 17 & 30 & 73.3 & 70.1 & 75.5 & -5.4 \\
\hline
\end{tabular}

Hospital types: 1: non-teaching non-academic hospital, 2: teaching non-academic hospital, 3: academic teaching hospital

\section{Standardized rates per hospital type}

Figures 4.1 and 4.2 show standardized ERCS rates and successful intended VBAC rates, respectively, per hospital type. Figure 4.1 illustrates that a wider range of ERCS rates exists amongst academic teaching hospitals. Also, the hospitals with the highest ERCS rates appear to be amongst the non-academic non-teaching hospitals. Figure 4.2 shows that with regard to VBAC, hospital types are more similar in terms of intended VBAC success rates. The highest success rates appear to be amongst non-academic teaching hospitals. 


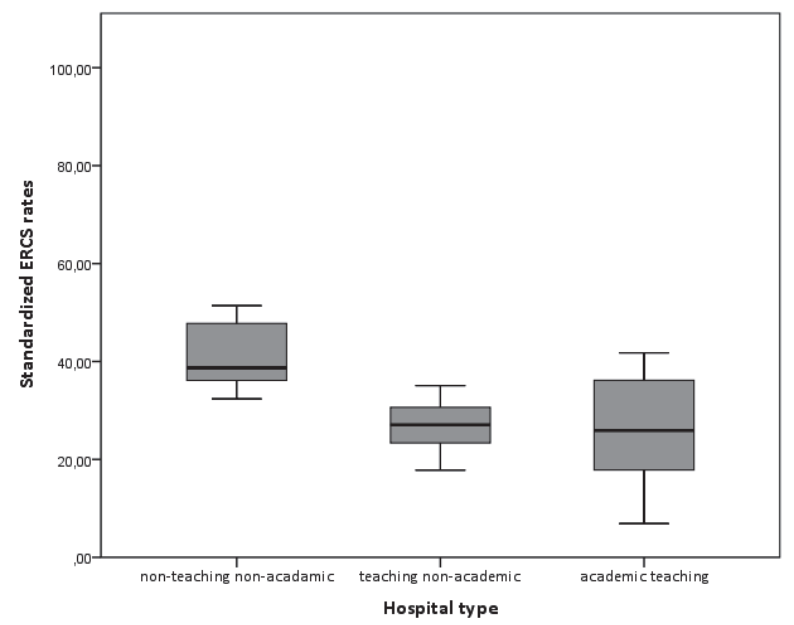

Figure 4.1 Standardized ERCS rate per hospital type.

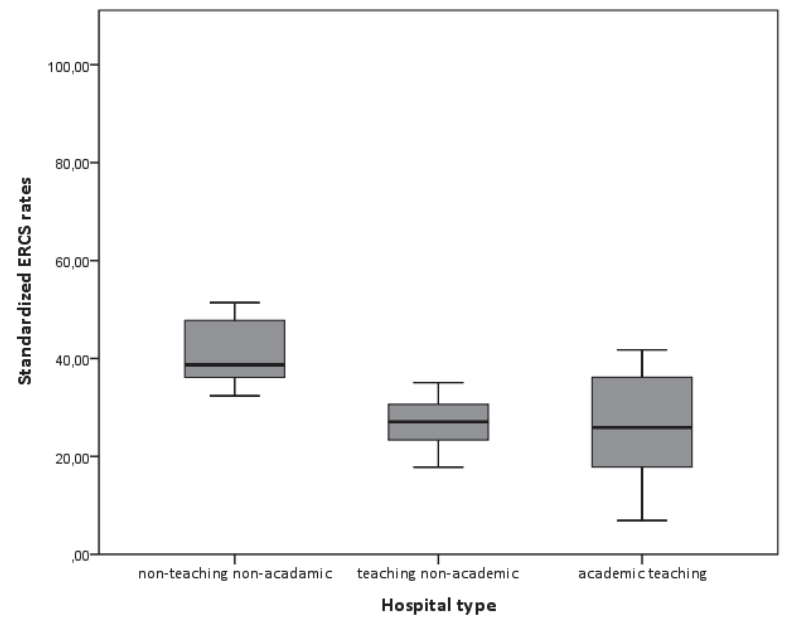

Figure 4.2 Standardized successful intended VBAC rate per hospital type.

\section{Correlation between ERCS and successful intended VBAC}

Since data was not normally distributed, correlation was tested using the Spearman's rank correlation test. The test for correlation between ERCS and successful intended VBAC resulted in a rho of -0.46 with a p-value of 0.065 showing that there was no correlation between successful intended VBAC and ERCS (Figure 4.3). 


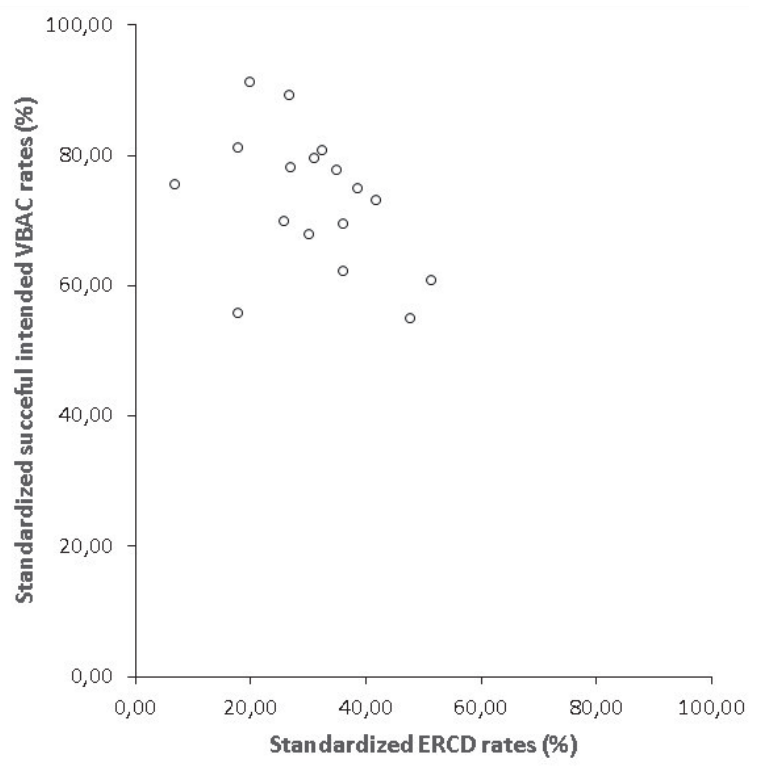

Figure 4.3 Correlation successful intended VBAC- and ERCS rates.

\section{Comment}

\section{Main findings}

In The Netherlands, the majority of women who are eligible for intended VBAC deliver vaginally. However, this study showed that in spite of its high mean VBAC rate, there is a striking amount of practice variation on mode of birth after CS in the Netherlands regardless of case mix. Hence, the a priori risk of having a CS independent of woman's individual risk factors varies per hospital. Amongst the 17 participating representative Dutch hospitals, ERCS rates corrected for case mix even varied between $6.0 \%$ and $54.0 \%$. For successful intended VBAC, this variation was less distinct but still $52.7 \%$ to $90.0 \%$. Yet, we showed that correcting for case mix only partly explained the observed practice variation. Accordingly, we can conclude that variables other than patient characteristics, like factors on hospital or provider level, play a more significant role in the development of practice variation. When estimating the effect of hospital type, it appeared that the largest variation exists amongst non-academic teaching hospitals. Also we showed that, although one could think that better risk selection leading in hospitals with higher ERCS rates would lead to lower emergency CS rates and thus higher successful intended VBAC rates, we showed that no correlation was found. 


\section{Strengths and weaknesses}

A strength of this study is that this is the first study to examine practice variation in vaginal birth after CS in a European country with correcting for case mix. We compared different levels of hospitals, from tertiary to small rural hospitals. The distribution of the different levels of hospitals is representative for the national distribution. Another strength is that data-selection occurred systematically by checking every single patient. In addition, the selection process of predictive variables for successful intended VBAC was in line with contemporary insights within prediction research. ${ }^{10,11}$ We chose to correct only for predictive variables for successful intended VBAC as every woman should be counselled the same way. Furthermore, this study provides insight in on which level possible interventions would be meaningful.

A drawback of the chosen methodology for predictor selection is that it implies the risk of missing contributing predictors. Hence, we assume that the developed models are capable of explaining practice variation and that the remaining variation can be attributed to policy. Yet, the remaining variation could also be explained by an incomplete model. For example, the predictor 'thickness of lower uterine segment' appears to be a probable independent predictor for failed intended VBAC but was not taken into account during data-collection. ${ }^{14}$ Another limitation is that, due to lack of data, we could not further specify the exact amount of practice variation that is derived from factors on hospital- and provider level. However, by performing an additional analysis in which we clustered types of hospital we were able to mainly visualize variation on hospital-level. Still, this implies the assumption that hospitals within the clusters are comparable and is therefore less precise.

\section{Interpretation}

In line with other studies, our study showed a large variation in intended VBAC rates between different hospitals. ${ }^{2,9}$ Large variation in intended VBAC rates is seen between countries, but at a national level there is large variation as well. ${ }^{2}$ Even in a small country with high intended VBAC rates as The Netherlands there is a remarkable amount of practice variation, comparable to countries with lower intended VBAC rates. ${ }^{2}$ Our results are in line with the cohort study of Kwee et al. ${ }^{15}$ In this study, performed in The Netherlands, practice variation was studied in 38 hospitals and showed a variation in intended VBAC from 46 to $87 \% .{ }^{15}$

The review of Guise et al reports a range from $28-70 \%$ in the US2 intended VBAC was more often performed in hospitals with a high birth-rate which are tertiary and teaching hospitals. Besides differences in attempting intended VBAC, there is a large variation in VBAC as well from 52 to $85 \%$ in the US. However, the review of Guise included mainly studies performed in academic- and non-academic teaching hospitals. ${ }^{2}$ Hence, this may 
cause a distorted view and the results may not be representative for rural hospitals, because retrospective studies show intended VBAC is less attempted in small rural hospitals. ${ }^{16}$ Our results confirm these findings and show 33-54\% in ERCS-rate in rural hospitals compared to $6-41 \%$ in academic- and non-academic teaching hospitals.

Toohill et al. ${ }^{9}$ distinguished hospitals based on a public or private setting and birth rates in Australia. This study revealed that women attempting intended VBAC in a public hospital with high birthrates have a higher chance of a successful intended VBAC. These findings are also similar to our results regarding the association between high birth rates and high successful intended VBAC.

For predicting successful intended VBAC, induction of labour was included as variable because it is known as an independent predictor. ${ }^{17,18}$ However, there might be practice variation in offering induction to a woman pregnant after CS as well. Yet, there is a lack of evidence to confirm this.

We can debate whether the observed ERCS rate is appropriate in relation to the type and abilities of specific hospitals. From the viewpoint of feasibility, it is understandable that obstetricians in small rural hospitals rather perform an ERCS than an intended VBAC as obstetric and theatre staffs are not on hand 24/7. In the United States guidelines even advice that hospitals attempting intended VBAC require equipment and staff able to perform an emergency CS immediately. ${ }^{2}$

At the same time, differences in the doctor's perspective can cause practice variation as well. In a tertiary hospital doctors could feel more comfortable in performing intended VBACs because they have more experience. Furthermore, doctors have to cope with an increased risk of liability, which may result in a decreased performance of intended VBAC. ${ }^{2}$ These factors may influence or induce practice variation as well.

In our study we chose to perform unilevel multivariate logistic regression analysis, because we assumed practice variation correlates with case mix. Future research could focus on multiple levels.

Overall, practice variation in mode of section after prior CS can only partly be explained by case mix. Besides effects of availability of medical staff and level of experience, methods of counselling and shared decision making may play a role as well, despite the guidelines. The practice variation resulting from this can probably be reduced and the quality of healthcare can be enhanced by more structured general information. We developed a decision aid and a prediction model to calculate the probability of a patient having a successful intended VBAC. By implementing this decision aid and model in daily healthcare, and informing women about their chances and the risks, and benefits of both options in a structured way, we anticipate reducing practice variation on patient level, provider level and hospital level. 
In conclusion variation between hospitals is large and can only for the smaller part be explained by case mix which indicates that local policy plays a more important role in the derivation of practice variation. Hence in order to enhance guideline implementation and to reduce practice variation, further research in for example the benefit of better counselling and shared decision making by using the developed prediction model and decision aid is essential.

\section{References}

1. WHO. A declaration on the promotion of patient's rights in Europe.1994.

2. Guise JM, Eden K, Emeis C, et al. Vaginal birth after caesarean: new insights. Evid Rep Technol Assess 2010; (191): 1-397.

3. https://www.healthypeople.gov/ 09.11.2014

4. NVOG - richtlijn Zwangerschap en bevalling na een voorgaande section caesarea. 2010 (www.nvog.nl).

5. RCOG. Birth after previous caesarean birth. Green-top Guideline. 2007;No. 45.

6. SOGC. Guidelines for Vaginal Birth After Previous Caesarean Birth. J Obstet Gynaecol Can. 2005(155):89: 319-31.

7. ACOG. Vaginal birth after previous caesarean section. ACOG practice bulletin. 2010(no. 115).

8. National Institute for Health and Clinical Excellence. Clinical Guideline 132: Caesarean Section. London: RCOG, 2011.

9. Toohill J, Gamble J, Creedy DK. A critical review of vaginal birth rates after a primary Caesarean in Queensland hospitals. Aust Health Rev 2013; 37(5): 642-8.

10. Harrell FJ. Regression Modeling Strategies. New York NS.

11. Steyerberg EW, Moons KG, van der Windt DA, et al. Prognosis Research Strategy (PROGRESS) 3: prognostic model research. PLoS Med 2013; 10(2): e1001381.

12. Donders AR, van der Heijden GJ, Stijnen T, Moons KG. Review: a gentle introduction to imputation of missing values. J Clin Epidemiol 2006; 59(10): 1087-91.

13. Steyerberg EW. Clinical Prediction Models. New York NS.

14. Kok N, Wiersma IC, Opmeer BC, de Graaf IM, Mol BW, Pajkrt E. Sonographic measurement of lower uterine segment thickness to predict uterine rupture during a trial of labour in women with previous Caesarean section: a meta-analysis. Ultrasound Obstet Gynecol 2013; 42(2): 132-9.

15. Kwee A, Bots ML, Visser GH, Bruinse HW. Obstetric management and outcome of pregnancy in women with a history of caesarean section in the Netherlands. Eur J Obstet Gynecol Reprod Biol 2007; 132(2): 171-6.

16. Goldman G, Pineault R, Bilodeau H, Blais R. Effects of patient, physician and hospital characteristics on the likelihood of vaginal birth after previous caesarean section in Quebec. CMAJ 1990; 143(10): 1017-24.

17. Landon MB, Leindecker S, Spong CY, et al. The MFMU Caesarean Registry: factors affecting the success of trial of labour after previous caesarean section. Am J Obstet Gynecol 2005; 193(3 Pt 2): 1016-23.

18. Tan PC, Subramaniam RN, Omar SZ. Predictors for caesarean section and neonatal admission after trial of labour in women with one previous lower segment caesarean scar. Singapore Med J 2008; 49(3): 188-92. 


\section{Part II}

Improving current practice on pregnancy following caesarean section 


\section{Chapter}

Predicting successful intended vaginal delivery after previous caesarean section: external validation of two predictive models in a Dutch nationwide registration-based cohort with a high intended vaginal delivery rate

ENC Schoorel, S Melman, SMJ van Kuijk, WA Grobman, A Kwee, BWJ Mol, JG Nijhuis, LJM Smits, R Aardenburg, K de Boer, FMC Delemarre, IM van Dooren, MTM Franssen, G Kleiverda, M Kaplan, SMI Kuppens, FTH Lim, JM Sikkema, E Smid-Koopman, H Visser, FPJM Vrouenraets, M Woiski, RPMG Hermens, HCJ Scheepers BJOG: An international journal of Obstetrics and Gynaecology 2014;121:840-7 


\section{Abstract}

\section{Objective}

To externally validate two models from the United States (entry-to-care (ETC) and close-todelivery (CTD)) that predict successful intended vaginal birth after caesarean (VBAC) for the Dutch population.

\section{Design and setting}

A nationwide registration-based cohort study. Seventeen hospitals in the Netherlands.

\section{Population}

Seven hundred and sixty-three pregnant women, each with one previous caesarean section (CS) and a viable singleton cephalic pregnancy without a contraindication for an intended VBAC.

\section{Methods}

The ETC model comprises the variables maternal age, pre-pregnancy body mass index (BMI), ethnicity, prior vaginal delivery, prior VBAC and prior nonprogressive labour. The CTD model replaces pre-pregnancy BMI with third-trimester BMI and adds estimated gestational age at delivery, hypertensive disease of pregnancy, cervical examination and induction of labour. We included consecutive medical records of eligible women who delivered in 2010. For validation, individual probabilities of women who had an intended VBAC were calculated.

\section{Main outcome measures}

Discriminative performance was assessed with the area under the curve (AUC) of the receiver operating characteristic and predictive performance was assessed with calibration plots and the Hosmer-Lemeshow (H-L) statistic.

\section{Results}

Five hundred and fifteen (67\%) of the 763 women had an intended VBAC; $72 \%$ of these (371) had an actual VBAC. The AUCs of the ETC and CTD models were 68\% (95\% CI 63-72\%) and 72\% (95\% CI 67-76\%), respectively. The H-L statistic showed a $P$-value of 0.167 for the ETC model and $P=0.356$ for the CTD model, indicating no lack of fit.

\section{Conclusion}

External validation of two predictive models developed in the United States revealed an adequate performance within the Dutch population. 


\section{Introduction}

After a first caesarean section (CS), a pregnant woman can opt for an elective repeat CS (ERCS) or an intended vaginal birth after caesarean (VBAC) (i.e. a trial of labour), which will result in an actual (successful) VBAC or an emergency CS (unsuccessful VBAC). Discussing the risks of both options is a substantial part of counselling on mode of delivery, and obviously the probability of having an actual VBAC is a key component. ${ }^{1,2}$ Published success rates for VBAC worldwide vary between 60 and $80 \% .^{3}$ However, these rates are not necessarily applicable for counselling, since individual probabilities may vary due to factors relating to demography, obstetric history and current pregnancy of the woman. ${ }^{3,4}$ Hence, a personalised prediction of VBAC may lead to a more refined counselling. Furthermore, with regard to clinical outcomes, personalised prediction could contribute to risk estimation because actual incidences of major maternal morbidity are lowest in women who have a $\operatorname{VBAC}(0.2 \%)$, followed by women having an ERCS $(0.8 \%)$, and are highest in women having unsuccessful VBAC (3.8\%). ${ }^{5}$ In addition, several studies have shown that low probabilities of successful VBAC are related to relatively high risks of major feto-maternal morbidity. ${ }^{6,7}$ Several scoring models that aim for a personalised prediction of successful intended VBAC have been published. ${ }^{3,8}$ In this work, the predictive models of Grobman et al. ${ }^{9}, 10$ are evaluated. These models can be used early in pregnancy ${ }^{9}$ and at the onset of labour ${ }^{10}$ in order to estimate the probability of successful intended VBAC during an at term delivery. Both models have previously been successfully validated in an independent cohort in the United States and were called the 'entry-to-care model' (ETC) and the 'close-todelivery model' (CTD). ${ }^{11,12}$ Additionally, the ETC model has been successfully validated for a Japanese population. ${ }^{13}$ However, differences in, for example, population characteristics and setting may affect the validity of the predictive models in European countries like the Netherlands. For instance, in the United States, the VBAC rate when the predictive models were derived was $12-22 \% ;{ }^{14}$ this declined to approximately $8.3 \%$ in $2007 . .^{15}$ In most European countries reported VBAC rates are higher, for example $54 \%$ in the Netherlands ${ }^{16}$ and $30-37 \%$ in the United Kingdom. ${ }^{2,17}$ Hence, in this study we aim to externally validate the prediction models of Grobman et al. ${ }^{9,10}$ for the Dutch population.

\section{Methods}

\section{Setting}

This nationwide registration-based cohort study was performed in 17 hospitals in the Netherlands, with a good representation of all geographic regions and hospital types. Hospitals types included university teaching hospitals $(n=5)$, nonuniversity teaching 
hospitals $(n=7)$ and nonuniversity nonteaching hospitals $(n=5)$. Approval for this study was obtained at the Medical Ethical Committee (CMO) of Maastricht University Medical Centre+ (MUMC+) (MEC number 09-4-047-13).

\section{Population}

The two predictive models were designed for women who have a vertex singleton pregnancy and a history of one low-transverse CS and who delivered at term (gestational age $\geq 37$ weeks), therefore only women who met these criteria were included in the present study. As in the studies of Grobman et al., ${ }^{9,10}$ women with an unknown indication for previous CS, an antepartum intrauterine fetal demise or a contraindication for vaginal delivery were excluded. Contraindications for vaginal delivery were defined as placenta praevia and a uterine scar with extension into the fundus.

Since we expected a large difference between the VBAC rate in the United States and the current VBAC rate in the Netherlands, we collected data from women who had an intended VBAC and from women who had an ERCS. The main consideration was to estimate the current VBAC rate in the Netherlands and subsequently to fully evaluate the applicability of the models in the Dutch population by comparing the baseline characteristics of the intended VBAC group and the ERCS group.

\section{Sample size}

The sample size was calculated according to the 'rule of thumb' of at least ten events per variable in the predictive model. ${ }^{18}$ An event was defined as an unsuccessful intended VBAC. Based on previously published data, it was assumed that the percentage of unsuccessful intended VBACs in the Netherlands would be $24 \% .{ }^{16}$ As the predictive models contained as many as 12 variables per model, the calculated minimum sample size was 500 subjects $(12 \times 10 / 0.24)$.

\section{Data collection}

At all participating sites, data were extracted from consecutive birth records according to a standardized operating procedure by using customized case report forms. Information was obtained on all predictive indicators included in the predictive models. Data were obtained by trained research nurses, medical doctors or senior medical students. In order to achieve the required sample size, each participating hospital was asked to include 30 consecutive cases of intended VBAC and all ERCSs in the same time interval, starting from 1 January 2010 . 


\section{Variables}

Variables were defined as described in the original articles of Grobman et al., ${ }^{910}$ The outcome variable used for validating the predictive models was the outcome of the intended VBAC, i.e. either successful intended VBAC (vaginal birth) or unsuccessful intended VBAC (emergency CS).

To be able to incorporate all variables despite the different units and definitions used in the two countries, some of the collected data had to be converted or redefined. All decisions on the conversion and redefinition of variables were approved by both a Dutch and an American obstetrician (HS and WG). The variables that had to be adapted were 'ethnicity' (in the United States the categories were African-American/Hispanic/White and others), 'fetal station' and 'cervical effacement'. In the Netherlands seven categories of ethnicity are used (Dutch, other European, Mediterranean, African, Indo-surinamese, Asian and 'other'). To correspond to the categories in the original prediction models, the variable 'AfricanAmerican' was set equal to the Dutch variable 'African'. The variable 'Hispanic', did not match any of the Dutch categories and was therefore abolished. Subsequently, the variable 'White and others' comprised all Dutch ethnicity categories except for 'African'. The variable 'fetal station' was collected according to the 'Hodge classification system', which ranges from Hodge $0(\mathrm{H} 0)$ to Hodge $4(\mathrm{H} 4)$. Fetal station was converted into the American classification system, which ranges from ballottable (B) to +5 . It was redefined as follows: $\mathrm{H} 0=-5 ; \mathrm{H} 1=-3 ; \mathrm{H} 2=-1 ; \mathrm{H} 3=0 ; \mathrm{H} 4=+3$. The variable 'cervical effacement' was collected in three categories, namely (1) $\leq 25 \%$, (2) $25-50 \%$ and (3) $\geq 50 \%$, as these are the categories in which these data are registered in the Netherlands. For analysis we set category 1 equal to $20 \%$, category 2 equal to $40 \%$ and category 3 equal to $75 \%$. Furthermore, the variable 'third trimester body mass index (BMI)' was approximated by adding $15 \mathrm{~kg}$ to 'pre-pregnancy weight'; this was considered appropriate for the Dutch population based on expert opinion.

\section{Data quality and missing data}

Data were entered and checked for inconsistencies. Inconsistent and incomplete data were double-checked directly with the hospital concerned. As shown in Table 5.1, for most variables there was only a small quantity of missing data. However, pre-pregnancy BMI was missing in $24 \%$ of women. A multiple imputation strategy was used for data analysis, since complete case analysis alone can result in a large loss of power and might yield biased parameter estimates. 


\section{Data analysis}

\section{Study cohort characteristics}

Characteristics of the women who had an ERCS and women who had an intended VBAC were compared. To compare proportions, the chi-squared test, or when appropriate Fisher's exact test, was used. For continuous variables, an independent sample $t$ test was used for all samples as data were normally distributed. A $P$-value $<0.05$ was used to indicate statistical significance.

\section{Predicted probabilities}

To validate the prediction models, for each patient who had an intended VBAC an individual probability of achieving VBAC was calculated with the following prediction equations obtained from the research articles of Grobman et al::9,10

1. The ETC model: ${ }^{9}$

$\exp (w) /[1+\exp (w)]$

where

$w=3.766-0.039$ (age, years) -0.060 (pre-pregnancy BMI)-0.671 (African-American ethnicity) -0.680 (Hispanic ethnicity) +0.888 (previous vaginal delivery) +1.003 (vaginal delivery after previous CS) -0.632 (previous CS due to nonprogressive labour).

2. The CTD model: ${ }^{10}$

$\exp (w) /[1+\exp (w)]$

where

$w=7.059-0.037$ (age, years) -0.044 (third-trimester BMI)-0.460 (African-American ethnicity) -0.761 (Hispanic ethnicity) +0.955 (previous vaginal delivery) +0.851 (vaginal delivery after previous CS) -0.655 (previous CS due to nonprogressive labour) -0.109 (estimated gestational age at delivery) -0.499 (hypertensive disease of pregnancy) +0.044 (cervical effacement, deciles) +0.109 (cervical dilation, $0-6 \mathrm{~cm}$ ) +0.082 (fetal station, B to +5 , entered as 0 to 11$)-0.452$ (labour induction).

Additionally, using the ETC model we calculated the mean predicted probability of achieving VBAC for women in the ERCS group and compared it with the mean predicted probability in the intended VBAC group. The purpose of this comparison was to evaluate whether the variables of the predictive models are already being taken into account during counselling. As data were not normally distributed, the mean predicted probabilities of achieving VBAC were compared using a Mann-Whitney $U$ test. We only performed this analysis with the ETC model because the CTD model includes intrapartum factors and was therefore not applicable to the ERCS group. 


\section{Discriminative and predictive performance}

The discriminative performance of the predictive models was assessed using a receiver operating characteristic (ROC). The ROC was obtained by plotting sensitivity against 1-specificity. Sensitivity was defined as the fraction of VBACs that were correctly predicted for a particular cut-off point, whereas specificity was defined as the fraction of unsuccessful intended VBACs that were correctly predicted. The ability of the models to discriminate between women with a high and low probability of achieving a VBAC was assessed using the area under the curve (AUC) of the ROC. The AUC can vary between 0.5 and 1.0, in which a value of 0.5 represents no discriminative capacity and 1.0 represents excellent discriminative capacity.

The predictive performance of the models was assessed by a calibration curve. The calibration curve was computed to show the relation between predicted probability of achieving VBAC and the observed VBAC rate. To obtain these values, the predicted probability was categorized into quantiles. In each quantile, the mean predicted VBAC rate was calculated and plotted against the observed VBAC rate in the corresponding quantile. In addition, to assessing goodness-of-fit, we computed the Hosmer-Lemeshow (H-L) statistic. The H-L statistic measures the fit of the calibration curve with the assumption (null hypothesis) that observed and predicted values are equal. A $P$-value $<0.05$ was considered to show lack of fit of the tested prediction models.

\section{Distribution of probabilities}

In order to determine the clinical utility of the models, we evaluated whether the model could classify a notable portion of women away from the VBAC population mean. Hence, we evaluated the distribution of probabilities amongst the cohort. The distributions were plotted in bar charts on the $x$-axis in the calibration plot. Additionally, we computed the percentage of the cohort that can be classified away from the VBAC population mean; we used cut-off values of $60 \%$ or less and $80 \%$ or higher.

\section{Software}

Statistical analyses and plots were performed using SPSS (SPSS v. 18.0) software and R, a language and environment for statistical computing.

\section{Results}

\section{Inclusions}

We reviewed 9833 consecutive medical records of women who had delivered in the participating hospitals since January 2010. One thousand and sixty-eight women (11\%) had a history of CS, 763 of whom (71\%) met the inclusion criteria. Of these 763 women eligible 
for intended VBAC, 515 (67\%) had an intended VBAC and 248 (33\%) had an ERCS. Three hundred and seventy-one women $(72 \%)$ delivered vaginally, resulting in an actual VBAC rate in our study cohort of $49 \%(371 / 763)$.

\section{Study cohort characteristics}

The population distributions with respect to the variables contained in the two predictive models are shown in Table 5.1. Women who had a previous vaginal delivery and/or a previous VBAC were more likely to attempt a VBAC. Women who had an intended VBAC also had a significantly lower BMI, although the actual difference between groups was small. On the other hand, women with a previous CS due to nonprogressive labour more often opted for ERCS. Women who had an ERCS delivered at a significantly lower gestational age. Based on the ETC model, women who chose a VBAC had a significantly higher mean predicted probability $(P<0.00)$ of successful intended VBAC $(72 \pm 14 \%)$ than women who chose an ERCS $(64 \pm 14 \%)$.

Table 5.1 Baseline characteristics of study cohort of women with a previous caesarean section.

\begin{tabular}{|c|c|c|c|c|}
\hline Variable & $\begin{array}{l}\text { Missing data intended } \\
\text { VBAC /ERCD }(n / n)\end{array}$ & $\begin{array}{l}\text { Intended VBAC } \\
\quad(n=515)\end{array}$ & $\begin{array}{c}\text { ERCS } \\
(n=248)\end{array}$ & P-value a \\
\hline Maternal age (years, mean \pm SD) & $2 / 4$ & $32 \pm 5$ & $33 \pm 4$ & 0.15 \\
\hline \multicolumn{5}{|l|}{ Ethnicity $(\mathrm{n},(\%))$} \\
\hline Dutch & $15 / 12$ & $388(75)$ & $192(77)$ & 0.53 \\
\hline Mediterranean & & $37(7)$ & $11(5)$ & 0.14 \\
\hline Other European & & $17(3)$ & $11(4)$ & 0.44 \\
\hline African & & $24(5)$ & $6(3)$ & 0.14 \\
\hline Indo-Surinamese & & $7(1)$ & $1(0)$ & 0.45 \\
\hline Asian & & $12(2)$ & $7(3)$ & 0.68 \\
\hline Other & & $15(3)$ & $8(3)$ & 0.81 \\
\hline Pre-pregnancy BMI $\left(\mathrm{kg} / \mathrm{m}^{2}\right.$, mean $\left.\pm \mathrm{SD}\right)$ & $124 / 79$ & $25 \pm 6$ & $27 \pm 7$ & $<0.00$ \\
\hline Previous CS due to failure to progress $(\mathrm{n},(\%))$ & $0 / 0$ & 201(39) & $135(54)$ & $<0.00$ \\
\hline Any previous vaginal delivery $(\mathrm{n},(\%))$ & $0 / 0$ & $127(25)$ & $25(10)$ & $<0.00$ \\
\hline Previous VBAC $(\mathrm{n},(\%))$ & $0 / 0$ & $99(19)$ & $8(3)$ & $<0.00$ \\
\hline PE/HELLP (n, (\%)) & $0 / 2$ & $9(2)$ & $6(2)$ & 0.58 \\
\hline Gestational age at delivery (days, mean \pm SD) & $0 / 0$ & $279 \pm 8$ & $273 \pm 9$ & $<0.00$ \\
\hline Cervical dilation $(\mathrm{cm}$, mean $\pm \mathrm{SD})$ & $11 / \mathrm{b}$ & $3 \pm 2$ & b & $\mathrm{b}$ \\
\hline Cervical effacement $(\%,($ mean $\pm \mathrm{SD}))$ & $56 /$ b & $64 \pm 19$ & $\mathrm{~b}$ & b \\
\hline Fetal station $(B,-5-+5$, mean $\pm S D)$ & $57 /$ b & $-2 \pm 2$ & $\mathrm{~b}$ & b \\
\hline Induction of labour $(\mathrm{n},(\%))$ & $0 / \mathrm{b}$ & $132(26)$ & $\mathrm{b}$ & $\mathrm{b}$ \\
\hline
\end{tabular}

${ }^{a}$ Results of $\chi^{2}$-tests/ Fisher's exact test / t-tests; ${ }^{b}$ Not applicable. SD: standard deviation, VBAC: vaginal birth after caesarean, ERCS: elective repeat caesarean section, CS: caesarean section, BMI: body mass index, PE: Preeclampsia HELLP syndrome: haemolysis, elevated liver-enzymes, low platelets, B: ballottement.

\section{Discriminative performance}

The discriminative performance of the predictive models is shown in Figure 5.1. The ROC of the ETC model has an AUC of $68 \%$ (95\% CI 63-72\%). The ROC of the CTD model has an AUC of $72 \%$ (95\% CI 67-76\%). 


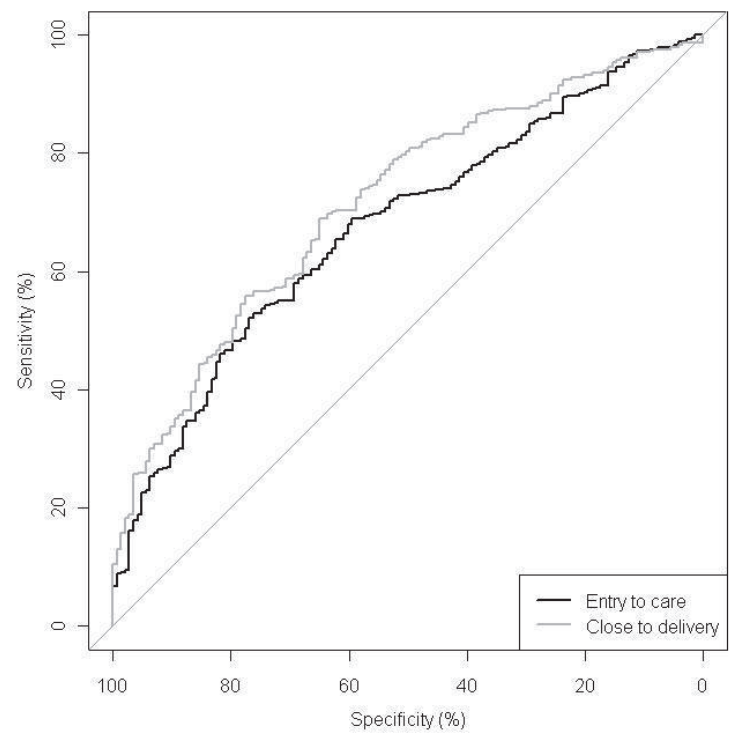

Figure 5.1 ROC of the entry-to-care model (AUC 68\%; 95\% CI 63-72\%) and the close-to-delivery model (AUC 72\%; 95\% CI 67-76\%), indicating the discriminative performance of both models concerning the probability of a successful vaginal birth after caesarean section.

\section{Predictive performance}

The overall calibration of both predictive models was good. The mean successful intended VBAC rate in this study cohort was $72 \%$. The mean predicted probabilities for successful intended VBAC in the ETC and the CTD models were $72 \pm 14 \%$ and $70 \pm 16 \%$, respectively. The predictive performances of the models are shown in the calibration curves in Figures 5.2 and 5.3 .

Both models show acceptable calibration; the calibration in the high-probability ranges was particularly good. The CTD shows better calibration than the ETC model. The H-L statistic showed a $P$-value of 0.17 for the ETC model and 0.36 for the CTD model, which indicates reasonable calibration of both predictive models.

\section{Distribution of probabilities}

The bar charts on the $x$-axes of Figures 5.2 and 5.3 show the distribution of probabilities of successful VBAC amongst the cohort. Figure 5.2 shows that when the ETC model is applied, the majority of the cohort has a predicted probability around or above the VBAC population mean of $60-80 \%$. In our cohort, $27 \%$ had a predicted probability above the VBAC population mean as these women had a predicted probability of $80 \%$ or higher. Furthermore, $19 \%$ of the women had a predicted probability below $60 \%$. As shown in 
Figure 5.3, with application of the CTD model the distribution of predicted probabilities is also concentrated around and above the VBAC population mean. In total, 31\% of women had a predicted probability above $80 \%$, and $26 \%$ had a predicted probability below $60 \%$.

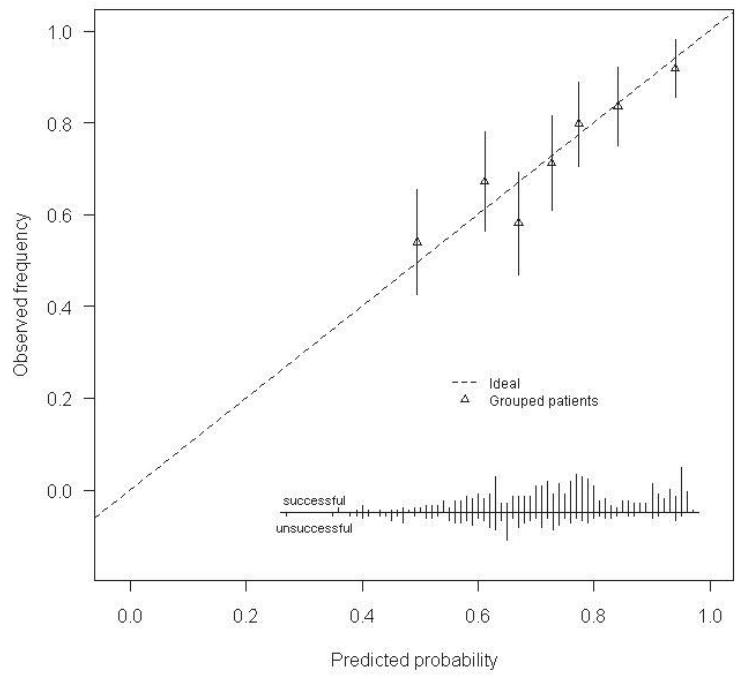

Figure 5.2 Calibration plot of the entry-to-care model with the observed frequency of a successful vaginal birth after caesarean section by the predicted probability. The triangles indicate quantiles of women with a similar predicted probability of success.

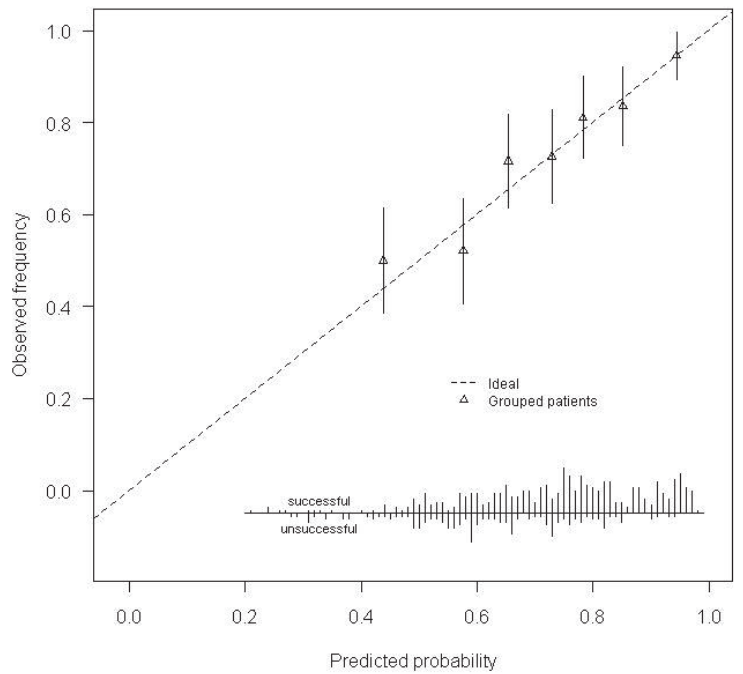

Figure 5.3 Calibration plot of the close-to-delivery model with the observed frequency of a successful vaginal birth after caesarean section by the predicted probability. The triangles indicate quantiles of women with a similar predicted probability of success. 


\section{Comment}

\section{Main findings}

External validation of two predictive models developed in the United States revealed adequate performance of both models within the Dutch population. Although overall calibration was acceptable, it was particularly good in the range of high predicted probability of successful intended VBAC. Discriminative capacity was reasonable for both models. Most women had a score within the population mean of $60-80 \%,{ }^{3}$ yet a notable minority was classified away from this population mean. Further, this study shows that in the Netherlands intended VBAC is still common practice, as shown by the intended VBAC rate of $67 \%$. Our results also suggest that preselection already occurs to some extent without applying a model.

\section{Interpretation}

According to guidelines on prognostic research, even a predictive model that seems promising requires external validation in different populations and settings. ${ }^{19}$ Ethnicity and the probability of attempting VBAC were the main observed differences between the Dutch and American settings, although other factors like intrapartum policy may also exist. We consider our results to be roughly generalizable to most other western European countries with comparable ethnicities and VBAC rates. External validation in our Dutch cohort showed some loss of discriminative performance, as the original AUCs were $75 \%$ (95\% CI $74-77 \%$ ) instead of $68 \%$ (95\% CI 63-72\%) for the ETC model and 77\% (95\% CI 76-78\%) instead of and $72 \%(95 \%$ CI $67-76 \%)$ for the CTD model. As shown, this finding was more pronounced for the ETC model. These findings are consistent with the previous validation studies performed in an American cohort by Costantine et al. ${ }^{11,12}$ who used a validation method comparable to our study. However, the results contrast with the findings in a Japanese cohort where an AUC of $80 \%(95 \% \text { CI } 72-89 \%)^{13}$ was obtained. However, because no information was provided in that article on variable conversion and there appeared to be an additional selection criterion regarding whether women were actually in labour, no actual comparison with our results could be made.

A review by Kaimal et al. ${ }^{20}$ highlighted that most women would like to be involved in decision-making about mode of birth. ${ }^{20}$ Also, women expressed their wish for personalised information. ${ }^{20}$ Hence, implementation of a predictive model could provide this tailored information by allowing estimation of the risk of emergency CS and the related risk of fetomaternal morbidity. ${ }^{6,7}$ The ideal predictive model would distinguish between a successful intended VBAC and a failed intended VBAC by polarising the cohort into two groups: women with a very high predicted probability and women with a very low predicted 
probability of achieving a VBAC. In comparison with other models that predict successful VBAC, the performance of the ETC and CTD models is average to good. ${ }^{4,8}$ However, for decision-making about mode of delivery after previous CS we consider it helpful to also distinguish women with a high or low probability of VBAC from those with an average probability. The ETC and CTD models show the potential to classify a notable portion of women away from the population mean, which might induce better a distribution with regard to risk classification of women amongst intended VBAC and ERCS. Therefore we think that a predictive model could not only contribute to more personalised counselling but also to a reduction in feto-maternal morbidity. However, the actual usefulness of such a model in terms of usability, applicability, change in birth preferences and feto-maternal morbidity should be further explored in a randomised controlled trial.

\section{Strengths and weaknesses}

A strength of this study is that it was performed in a multicentre setting with a good representation of types of hospitals and geographic regions in the Netherlands, which increased the external validity of our results. Furthermore, our data collection provides insight into the current (intended) VBAC rates in the Netherlands and into the prognostic profiles of women who opt for an intended VBAC and ERCS. By performing classification analysis we are able to show the subgroup of women who will have a probability of VBAC that is different from current population means. ${ }^{1,2}$

We also recognise some limitations to our study. Firstly, there was a possible loss of discriminative performance of the validated models due to the necessary redefinition and conversion of variables into Dutch units. We had to redefine fetal station from a scale consisting of 12 steps ranging from B to -5 to +5 to the Dutch scale that consists of five steps and ranges from 0 to 4 . Redefinition could induce misclassification and loss of refinement within variables, and thereby compromise model performance. Furthermore, in both models ethnicity is an important predictor. Though ethnicity has been recognized as an important demographic factor with regard to the probability of successful VBAC, ${ }^{4}$ the underlying mechanism is unknown and might be influenced by socioeconomic factors. Therefore, the ethnicity categories might not be compatible in other settings. Hence, we recommend re-estimation of ethnicity and the intrapartum variables before application of the models in an impact study or clinical practice. A second drawback is that we had to approximate the variable 'third trimester BMI' as it could not be obtained from the charts. This may have led to imprecision and impairment of the performance of the models. Thirdly, the models would ideally be evaluated through application in a prospective setting. Application of the models might induce different birth preferences in women, selecting women with more favourable prognostic profiles for successful VBAC. This might alter 
model performance. Furthermore, a limitation with regard to the validated models that must be addressed concerns the timing of counselling. We consider that, from a medical point of view, counselling on mode of delivery should ideally occur in the third trimester of pregnancy, because then other factors can be incorporated that are known in late pregnancy such as estimated fetal weight (EFW) and whether labour needs to be induced. The ETC model does not take these factors into account whilst the CTD model is applicable when there is an indication for induction of labour or when labour has already started. In this regard, in terms of practical use, a predictive model that can be used in the third trimester would be more suitable for the Dutch setting.

\section{Conclusion}

External validation of two predictive models developed in the United States revealed adequate performance of both models within the Dutch setting. The predictive models can classify a notable portion of women away from the VBAC population mean. However, whether women indeed perceive the information on probability of successful VBAC as useful and whether the models hold when applied in a prospective setting should be additionally evaluated. Additional redefinition of the 'ethnicity' variable for a western European setting and transformation of both models into one model for third-trimester counselling could enhance model performance and increase applicability to the Dutch setting.

\section{References}

1. ACOG Practice bulletin no. 115: Vaginal birth after previous cesarean delivery. Obstet Gynecol 2010; 116(2 Pt 1): $450-63$.

2. RCOG Green-top Guideline no. 45: Birth after previous caesarean birth. RCOG Press; 2007.

3. Grobman WA. Rates and prediction of successful vaginal birth after cesarean. Semin Perinatol 2010; 34(4): 244-8.

4. Guise JM, Denman MA, Emeis C, et al. Vaginal birth after cesarean: new insights on maternal and neonatal outcomes. Obstet Gynecol 2010; 115(6): 1267-78.

5. McMahon MJ, Luther ER, Bowes WA, Jr., Olshan AF. Comparison of a trial of labor with an elective second cesarean section. N Engl J Med 1996; 335(10): 689-95.

6. Grobman WA, Lai Y, Landon MB, et al. Can a prediction model for vaginal birth after cesarean also predict the probability of morbidity related to a trial of labor? Am J Obstet Gynecol 2009; 200 (1): 56 e1-6.

7. Chaillet N, Bujold E, Dube E, Grobman WA. Validation of a prediction model for predicting the probability of morbidity related to a trial of labour in quebec. J Obstet Gynaecol Can 2012; 34(9): 820-5.

8. Eden $\mathrm{KB}$, McDonagh M, Denman MA, et al. New insights on vaginal birth after cesarean: can it be predicted? Obstet Gynecol 2010; 116(4): 967-81.

9. Grobman WA, Lai Y, Landon MB, et al. Development of a nomogram for prediction of vaginal birth after cesarean delivery. Obstet Gynecol 2007; 109(4): 806-12. 
10. Grobman WA, Lai Y, Landon MB, et al. Does information available at admission for delivery improve prediction of vaginal birth after cesarean? Am J Perinatol 2009; 26(10): 693-701.

11. Costantine MM, Fox K, Byers BD, et al. Validation of the prediction model for success of vaginal birth after cesarean delivery. Obstet Gynecol 2009; 114(5): 1029-33.

12. Costantine MM, Fox KA, Pacheco LD, et al. Does information available at delivery improve the accuracy of predicting vaginal birth after cesarean? Validation of the published models in an independent patient cohort. Am J Perinatol 2011; 28(4): 293-8.

13. Yokoi A, Ishikawa K, Miyazaki K, Yoshida K, Furuhashi M, Tamakoshi K. Validation of the prediction model for success of vaginal birth after cesarean delivery in Japanese women. Int J Med Sci 2012; 9(6): 488-91.

14. Menacker F, Declercq E, Macdorman MF. Cesarean delivery: background, trends, and epidemiology. Semin Perinatol 2006; 30(5): 235-41.

15. MacDorman M, Declercq E, Menacker F. Recent trends and patterns in cesarean and vaginal birth after cesarean (VBAC) deliveries in the United States. Clin Perinatol 2011; 38(2): 179-92.

16. Kwee A, Bots ML, Visser GH, Bruinse HW. Obstetric management and outcome of pregnancy in women with a history of caesarean section in the Netherlands. Eur J Obstet Gynecol Reprod Biol 2007; 132(2): 171-6.

17. Montgomery AA, Emmett CL, Fahey T, et al. Two decision aids for mode of delivery among women with previous caesarean section: randomised controlled trial. BMJ 2007; 334(7607): 1305.

18. Peduzzi P, Concato J, Kemper E, Holford TR, Feinstein AR. A simulation study of the number of events per variable in logistic regression analysis. J Clin Epidemiol 1996; 49(12): 1373-9.

19. Steyerberg EW, Moons KG, van der Windt DA, et al. Prognosis Research Strategy (PROGRESS) 3: prognostic model research. PLoS Med 2013; 10(2): e1001381.

20. Kaimal AJ, Kuppermann M. Understanding risk, patient and provider preferences, and obstetrical decision making: approach to delivery after cesarean. Semin Perinatol 2010; 34(5): 331-6. 


\section{Chapter 6}

\section{Vaginal birth after a caesarean section: the development of a Western European population based prediction model for deliveries at term}

ENC Schoorel, SMJ van Kuijk, S Melman, JG Nijhuis, LJM Smits, R Aardenburg, K de Boer, FMC Delemarre, IM van Dooren, MTM Franssen, M Kaplan, G Kleiverda, SMI Kuppens, A Kwee, FTH Lim, BWJ Mol, FJME Roumen, JM Sikkema, E Smid-Koopman, H Visser, M Woiski, RPMG Hermens, HCJ Scheepers BJOG: An international journal of Obstetrics and Gynaecology 2014;121:194-201 


\section{Abstract}

\section{Objective}

To develop and internally validate a model that predicts the outcome of an intended vaginal birth after caesarean (VBAC) for a West European population which can be used to personalize counselling of deliveries at term.

\section{Design and setting}

Registration-based retrospective cohort study.

Five university teaching hospitals, seven non-university teaching hospitals and five nonuniversity non-teaching hospitals in The Netherlands.

\section{Population}

515 women with a history of one caesarean section, a viable singleton pregnancy without a contraindication for intended VBAC, who delivered at term.

\section{Methods}

Potential predictors for a vaginal delivery after caesarean section (CS) were chosen based on literature and expert opinions. We internally validated the prediction model using bootstrapping techniques.

\section{Main outcome measures}

Predictors for VBAC; for validation, the area under the receiver operating characteristic curve (AUC) for discriminative capacity and calibration-per-risk-quantile for accuracy.

\section{Results}

371 out of 515 women had a VBAC (72\%). Variables included in the model were: estimated fetal weight $\geq \mathrm{P} 90$ in the third trimester, previous non-progressive labour, previous vaginal delivery, induction of labour, pre-pregnancy body-mass-index and ethnicity. The AUC of receiver operating characteristic was $71 \% \quad(95 \%$ confidence interval $(\mathrm{CI})=69-73 \%)$, indicating good discriminative ability. The calibration plot shows that predicted probabilities are well calibrated, especially from $65 \%$ up, which accounts for $77 \%$ of the total study population.

\section{Conclusion}

We developed an appropriate Western-European population based prediction model that is aimed to personalize counselling of term deliveries. 


\section{Introduction}

In pregnancies following a caesarean section (CS), women should be offered counselling for both an intended vaginal birth after caesarean (VBAC) (i.e. trial of labour) and an elective repeat CS (ERCS). A substantial part of counselling is discussing the probability that an intended VBAC results in an actual vaginal delivery of a healthy child. ${ }^{1}$ In comparison to a $\mathrm{CS}$, a successful VBAC (i.e. actual vaginal delivery) is associated with fewer major complications $^{2}$, a shorter recovery period and high maternal satisfaction. ${ }^{3,4}$ However, if an intended VBAC results in an emergency CS, it is in comparison to an ERCS associated with a higher risk of major complications as hysterectomy and operative injury ${ }^{2}$ and with a relatively low maternal satisfaction. ${ }^{3,} 4$ Published success-rates of intended VBAC vary between $49 \%$ and $87 \% 0^{5}$ but it is evident that factors on patient, provider, and hospital level can influence the probability of success, and VBAC related morbidity. ${ }^{5-7}$ Hence, prediction of a woman's individual probability of successful intended VBAC is a potentially useful tool to aid the decision-making process. Although many prediction tools that predict successful or unsuccessful intended VBAC have been published over the past two decades ${ }^{5,6,8}$, only a few of these models are externally validated in other cohorts ${ }^{6-8}$, while impact studies have not been performed at all. Consequently, current guidelines on pregnancy and childbirth after CS still recommend counselling with the use of the overall probability of success, instead of using a personalized one. ${ }^{1}$ It is remarkable that the majority of the prediction models were developed in a non-European population. These models cannot be directly translated to European settings as differences in obstetrical policy and the entirely different mix of ethnicities may impair the performance and validity of the models. For the European setting, only one non-externally validated model was available ${ }^{7}$, and two models 9,10 developed in North-American cohorts were previously externally validated for a Dutch population by our researchgroup. ${ }^{11}$ However, these models are less applicable for counselling since they either include factors that are only known intrapartum ${ }^{7,10}$ or the variables have yet to be adjusted for a West European population. ${ }^{9}, 10$ Hence, this study aims to develop a new prediction model by combining and improving existing models which predicts successful intended VBAC more reliably, and which is suitable for a Western European population and can be applied as a tool to personalize counselling on mode of delivery after CS for deliveries at term. 


\section{Methods}

\section{Setting}

This registration-based retrospective cohort study was conducted in 17 Dutch hospitals which were involved in the Dutch research consortium for women's health (www.studiesobsgyn.nl. The enrolled hospitals covered all Dutch geographic regions. Included hospitals types were university teaching hospitals $(n=5)$, non-university teaching hospitals $(n=7)$ and non-university non-teaching hospitals $(n=5)$. Approval for this study was obtained at the Medical Ethical Committee (CMO) of Maastricht University Medical Centre+ (MUMC+) (MEC number 09-4-047-13).

\section{Population}

Women were included when they had a history of one previous CS with any number of prior vaginal deliveries and had a vertex singleton pregnancy as well as a delivery at term ( $\geq 37$ weeks gestational age (GA)) in their current pregnancy. We excluded women with an unknown indication of prior CS, or more than one previous CS, or in the current pregnancy either an intra uterine fetal demise or a contraindication for an intended VBAC. A contraindication for an intended VBAC was defined as a previous uterine rupture, a placenta praevia or a uterine scar with extension into the fundus.

\section{Potential predictors}

Contemporary methodological guidelines for prognostic modelling state that potential predictors should be preselected based on clinical reasoning and evidence from previous reports instead of observed significant relations with outcome variables in the same dataset. This method results into higher external validity and less overfitting of the developed model. ${ }^{12-14}$ Therefore, we preselected potential predictors based on previously published prediction models, expert opinions and articles reporting on risk factors for a successful intended VBAC.5,8-10 We preselected predictor variables from obstetrical history, medical history and demography of the patient. The final set of potential predictors that was considered for the model included estimated fetal weight (EFW), previous CS due to nonprogressive labour, any former vaginal birth, the occurrence of preeclampsia or the syndrome of haemolysis, elevated liver enzymes and low platelet count (HELLP) during current pregnancy, induction of labour in the current pregnancy, chronic and/or gestational hypertension, pre-pregnancy body mass index (BMI), maternal age and ethnicity. Nonprogressive labour was defined as arrest of descent, dilation or labour. If no pre-pregnancy BMI measurement was available, we used the routinely measured first trimester BMI. Ethnicity is reported as one of the strongest of the demographic predictors for successful intended $\mathrm{VBAC}^{15}$, with women of European descent having a higher probability of 
successful intended VBAC than women of Hispanic and African ethnicity. ${ }^{6}$ The underlying cause however, has remained unexplained. ${ }^{15}$ We hypothesized that the effect of ethnicity on successful VBAC might both be explained by socio-cultural aspects as well as by biological differences and could therefore vary between countries. As there was no available literature on the relation between ethnicity and VBAC for a Dutch or another West European population, we performed a univariate analysis to evaluate the association between different ethnicity strata and the probability of successful VBAC, using a liberal p-value of 0.20 . In The Netherlands, ethnicity is documented within seven predefined categories: Dutch, Mediterranean, other European, African, Asian, Indo-Surinamese, and 'other'. For the prediction model development we defined Caucasian ethnicity as Dutch or 'other European'.

Further, we aimed to incorporate EFW as a variable that is customized to gestational age in order to enable use of the model at any term within the third trimester of pregnancy. In The Netherlands, the curves of Snijders et al. $1994^{16}$ are often used which were established along the curve of Yudkin et al. ${ }^{17}$ Hence after calculation of the fetal weight using the Hadlock formula ${ }^{18}$, fetal weight was customized to gestational age. Subsequently, we chose to create two dummy variables, one indicating EFW is equal to or below the $10^{\text {th }}$ percentile, the other indicating that $\mathrm{EFW}$ is equal to or higher than the $90^{\text {th }}$ percentile $(\mathrm{P})$. We obtained $\mathrm{EFW}$ measurements performed in the third trimester between 33 and 37 weeks GA and measured by either ultrasound or abdominal palpation.

\section{Sample size}

There is consensus about the maximum number of predictors that can be validly included in a prediction model. It is recommended that at least ten events are collected for each potential predictor that is evaluated in the multivariable regression analysis. ${ }^{19}$ An event is defined as the least frequent outcome status, which is in our case an unsuccessful intended VBAC. In The Netherlands the estimated event rate, i.e. intended VBAC failure rate, is 24-28\%. ${ }^{11,20}$ Therefore, in order to develop a model with nine potential predictors, at least 90 events were required. Hence, a sample size of at least 321 subjects was required $(90 / 28 * 100)$.

\section{Data collection}

Patient data regarding demography of the patient and potential predictors regarding obstetrical and medical history were extracted from medical records according to a standardized operating procedure by using customized case report forms (CRF) at all participating sites. Data were obtained by trained research nurses, medical doctors or senior medical students. Subjects were consecutively selected and included from the hospitals' birth registers. To meet the required sample size, we requested each participating hospital to 
include 30 subsequent cases of intended VBAC that matched the inclusion criteria, starting at the first of January 2010 until 31 December 2010.

\section{Data quality and missing data}

Data were checked for completeness and inconsistencies. Inconsistent and incomplete data were double-checked directly with the hospital concerned. Values we were unable to collect were imputed using a multiple imputation strategy, since the omission of patients who have one or more predictor variables missing from the analysis can cause a considerable loss of precision and may bias results. ${ }^{12,21}$ The number of imputations was set to five. Subsequently, we performed a sensitivity analysis by comparing outcomes of the imputed dataset with the use of complete cases analysis only. The purpose of this analysis was to determine whether imputation led to radically different results or a different conclusion.

\section{Model development}

We used restricted cubic splines and graphs to determine whether continuous potential predictors could be analysed as linear terms. In each of the five imputed datasets, we introduced all of the potential predictors in a multivariable logistic regression model, using successful intended VBAC as the outcome variable. To reduce the amount of predictors in these models, we used backward stepwise deletion based on the Wald test. We used a liberal p-value of 0.20 , as recommended by prediction modelling guidelines. ${ }^{13}$ Predictors that remained in at least three out of five imputed datasets were included in the final model, and were re-estimated in all imputed datasets separately. Results of these five models were combined into a single prediction model. ${ }^{22}$

\section{Internal validation}

Stepwise selection methods such as backward stepwise deletion are often discouraged to use because they can introduce selection bias, since predictors that are by chance overestimated are more likely to be included than predictors that are by chance underestimated. This can result in a prediction model that may be overfitted to the derivation data, i.e. it performs well in the data it was derived on, but performance will degrade considerable when applied to future patients. Generally, predictions will be too extreme. In order to reduce the risk of overfitting, we internally validated the model using bootstrapping. In this internal validation step, $B$-bootstrap samples of the same size as the original sample (we used $B=1000$ ) were drawn with replacement from the original data, which reflects drawing of samples from the underlying population. This was performed to estimate the likely performance in future patients, and adjust the model so future predictions will be less extreme. 


\section{Performance of the model}

To assess the performance of the internally validated model, we quantified measures of discrimination and calibration. The models ability to discriminate between women who had a successful intended VBAC and women who had a failed intended VBAC (emergency CS), was quantified as the area under the receiver operating characteristic (ROC) curve. This ranges from $50 \%$ (no discriminative capacity) to $100 \%$ (perfect discriminative capacity). The agreement between predicted probabilities and observed frequencies of the outcome (accuracy) was assessed by visually inspecting the calibration plot. Furthermore, we computed the Hosmer and Lemeshow (H-L) goodness-of-fit statistic as a quantitative measure of accuracy. A high H-L statistic is related to a low p-value and indicates lack of fit. All statistical analyses were performed using SPSS version 20.0 and R version 2.12.2; http://www.r-project.org).

\section{Results}

\section{Patient population}

A total of 515 women with a history of one CS were identified as eligible for the present study. Baseline characteristics of the cohort are presented in Table 6.1. The overall successful intended VBAC rate was $72.0 \%$ (in total 371 women).

Table 6.1 Baseline characteristics of who attempt a vaginal birth after caesarean section.

\begin{tabular}{|c|c|c|}
\hline Characteristic & Missing values & Women intended VBAC N=515 \\
\hline Maternal age (years, mean \pm SD) & $2(0)$ & $32.2 \pm 5.0$ \\
\hline \multicolumn{3}{|l|}{ Ethnicity $(\mathrm{n},(\%))$} \\
\hline - Caucasian & & $405(81.0)$ \\
\hline - Mediterranean & & $37(7.4)$ \\
\hline - African & $15(3.0)$ & $24(4.8)$ \\
\hline - Asian & & $12(2.4)$ \\
\hline - Indo-Surinamese & & $7(1.4)$ \\
\hline - Other & & $15(3.0)$ \\
\hline Parity (mean $\pm \mathrm{SD})$ & $0(0)$ & $1.4 \pm 0.8$ \\
\hline Pre-pregnancy BMI $\left(\mathrm{kg} / \mathrm{m}^{2}\right.$, mean $\left.\pm \mathrm{SD}\right)$ & $124(24.1)$ & $25.3 \pm 5.7$ \\
\hline Previous CS due to non-progressive labour $(\mathrm{n},(\%))$ & $0(0)$ & $201(39.0)$ \\
\hline Any previous vaginal delivery $(\mathrm{n},(\%))$ & $0(0)$ & $127(24.7)$ \\
\hline Previous VBAC $(\mathrm{n},(\%))$ & $0(0)$ & $99(19.2)$ \\
\hline PE/HELLP & $0(0)$ & $9(1.7)$ \\
\hline Diabetes Mellitus & $0(0)$ & $13(2.5)$ \\
\hline Hypertension & $0(0)$ & $37(7.2)$ \\
\hline Induction of labour (n, $(\%))$ & $0(0)$ & $132(25.6)$ \\
\hline Estimated fetal weight $\leq \mathrm{p} 10$ & $201(39.0)$ & $26(8.3)$ \\
\hline Estimated fetal weight $\geq \mathrm{p} 90$ & $201(39.0)$ & $11(3.5)$ \\
\hline Estimated gestational age at delivery (weeks, mean \pm SD) & $0(0)$ & $39.8 \pm 1.2$ \\
\hline
\end{tabular}

VBAC: vaginal birth after caesarean, SD: standard deviation, BMI: body mass index, CS: caesarean section, PE: Preeclampsia HELLP syndrome: haemolysis, elevated liver-enzymes, low platelets, p: percentile. 


\section{Model development and internal validation}

The number of missing values per predictor variable are shown in Table 6.1. For the majority of the potential predictors, there was only a small amount of missing data. However, pre-pregnancy BMI was missing in $24 \%$ of women and estimated fetal weight (EFW) was missing in 39\% of women. After imputation, all 515 women were available for multivariable modelling. Subsequently, based on univariate analyses for the variable 'ethnicity', we selected Caucasian ethnicity (yes/no) as potential predictor ( $95 \%$ confidence interval (CI) odds ratio(OR) $0.92-2.32$, $\mathrm{p}$-value 0.11 ). We entered all potential predictors in the model and identified six predictors that met our selection criteria. These six predictors were: pre-pregnancy BMI, obstetrical history (previous vaginal delivery and previous nonprogressive labour), Caucasian ethnicity, induction of current labour and EFW $\geq \mathrm{P} 90$. These six variables were combined into one model. Maternal age and BMI are continuous variables; all other predictors are dichotomous variables. Table 6.2 shows the original prediction model (i.e. after variable selection and estimation of parameters in five imputed datasets) that estimates a successful intended VBAC. The bootstrap validation yielded a shrinkage factor of 0.78 , which was used to multiply the regression coefficients by. After the adjustment of regression coefficients, the intercept was re-estimated (Table 6.2).

Table 6.2 Prediction model for the estimation of a successful intended VBAC with regression coefficients and odds ratio's before and after internal validation.

\begin{tabular}{|c|c|c|c|c|}
\hline Variable & & $\begin{array}{c}\text { Regression coefficient } \\
\text { (crude) }\end{array}$ & $\begin{array}{c}\text { * Regression } \\
\text { coefficient (adjusted) }\end{array}$ & OR (crude) $(95 \% \mathrm{CI})$ \\
\hline Intercept & & 1.876 & 1.647 & - \\
\hline Caucasian & yes/no & 0.476 & 0.371 & $1.61(0.97-2.66)$ \\
\hline Pre-pregnancy BMI & $\mathrm{kg} / \mathrm{m}^{2}$ & -0.041 & -0.032 & $0.96(0.92-1.00)$ \\
\hline $\begin{array}{l}\text { Previous CS due to non- } \\
\text { progressive labour }\end{array}$ & yes/no & -0.688 & -0.537 & $0.50(0.33-0.76)$ \\
\hline Any previous vaginal delivery & yes/no & 1.339 & 1.045 & $3.81(2.10-6.92)$ \\
\hline Induction of labour & yes/no & -0.660 & -0.515 & $0.52(0.33-2.10)$ \\
\hline Estimated fetal weight $\geq \mathrm{p} 90$ & yes/no & -0.624 & -0.487 & $0.54(0.14-2.02)$ \\
\hline
\end{tabular}

* Regression coefficients after adjustment for overfitting by shrinkage (shrinkage factor $=0.78$ ), the intercept was re-estimated. To calculate the absolute risk of a successful intended VBAC: $\mathrm{P}_{\text {(success }}=1 /\left(1+\mathrm{e}^{\text {-(Linear part) }}\right) * 100 \%$. Linear part $=1.647+(0.371 *$ Caucasian $)-(0.032 *$ pre-pregnancyBMI $)-(0.537 *$ Previous_non-progressive_labour $)+(1.045$ $*$ Previous vaginal delivery $)-\left(0.515^{*}\right.$ Induction of labour $)-(0.487 * \mathrm{EFW} \geq \mathrm{p} 90)$. CI: confidence interval, BMI: body mass index, CS: caesarean section, PE: Preeclampsia HELLP syndrome: haemolysis, elevated liver enzymes, low platelets, p: percentile.

The final predictive equation that can be used to estimate the individual probability of a successful intended VBAC for future patients is: $\mathrm{P}_{\text {(success) }}=\left(1 /\left(1+\exp \left(-\left(1.647+0.371^{*}\right.\right.\right.\right.$ Caucasian- $0.032 *$ pre-pregnancy $\quad$ BMI-0.537*Previous non-progressive labour $+1.045^{*}$ Previous vaginal delivery- $0.515 *$ Induction of labour $-0.487 * \mathrm{EFW} \geq \mathrm{P} 90)))) * 100 \%$. For example, a woman of Caucasian ethnicity, a BMI of $26 \mathrm{~kg} / \mathrm{m}^{2}$, a previous CS due to non- 
progressive labour, no previous vaginal delivery, no induction of labour and the in the third trimester EFW is smaller than P90 has a predicted probability of successful intended VBAC of $65.7 \%$. When an indication for induction presents, the probability of this patient shifts to $53.3 \%$, this information can be used for a re-evaluation of chosen mode of birth.

\section{Performance of the model}

Discriminative performance of the prediction model is shown in figure 6.1. The area under the ROC curve (AUC) was 70.8\% (95\% confidence interval $(\mathrm{CI})=68.6-72.9 \%$ ), which indicates good discriminative ability. Calibration was good, indicated with a non-significant H-L statistic (0.13).

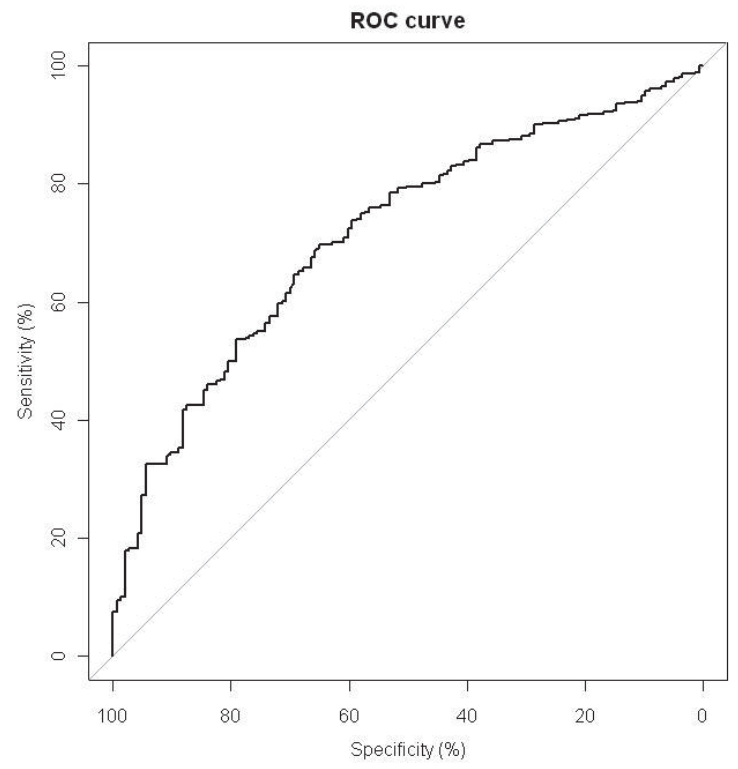

Figure 6.1 Receiver operating characteristic (ROC) of the prediction model (AUC: 70.8\%; 95\% CI: \% 68.6-72.9), indicating reasonable discriminative performance of the model.

The corresponding calibration curve that represents accuracy of the model is shown in Figure 6.2. Predicted probabilities ranged from $39 \%$ to $93 \%$, with a mean of $72 \%$ (SD: $11 \%$ ). Overall, the developed prediction model has a good fit to reference curve, i.e.. predicted probabilities agree observed probabilities. The calibration plot shows that predicted probabilities are especially well calibrated from about $65 \%$ up, which accounts for $77 \%$ of the target group. However, in women who have a probability of success of lower than $50 \%$, the model generally underestimates their probability of successful intended VBAC. 


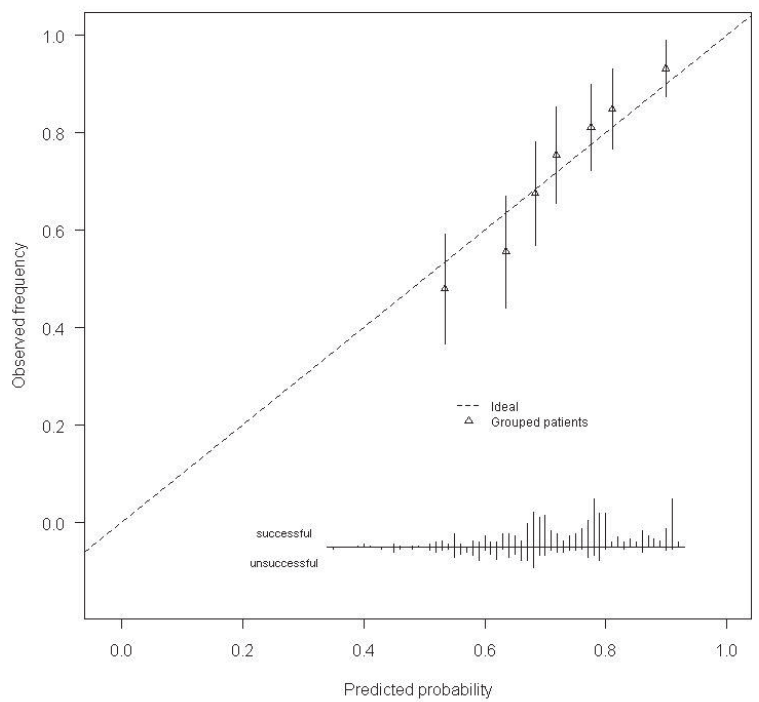

Figure 6.2 Calibration plot with the observed frequency of a successful intended vaginal birth after caesarean section by the predicted probability. The triangles indicate quantiles of women with a similar predicted probability of success.

\section{Discussion}

\section{Main findings}

In this study we developed and internally validated a prediction model in accordance with contemporary methodological guidelines in prognostic modelling. ${ }^{12-14}$ The prediction model estimates successful intended VBAC and is aimed for application for personalized counselling in the third trimester of pregnancy, i.e. for women with an expected term delivery. The final model for predicting successful intended VBAC consists of six variables including demographic variables (pre-pregnancy BMI, Caucasian ethnicity), obstetrical history (previous vaginal delivery and previous non-progressive labour) and current pregnancy variables (induction of current labour and EFW $\geq$ P90). The developed prediction model has reasonable discriminative capacity and is accurate, especially for women with a predicted probability of $65 \%$ and higher. In order to determine whether the amount of missing values within EFW and BMI led to different conclusions and/or results, we performed a sensitivity analysis by comparing outcomes of the imputed dataset with the use of complete cases analysis only. This analysis showed similar results for both datasets, therefore we concluded that imputation did not lead to radically different results. 


\section{Strengths and weaknesses}

A strong point of this study is that we obtained consecutive cases based on the hospitals' birth registers in various geographic regions and hospital types in The Netherlands. This method results into a representative, high quality dataset. Another strength is that we aimed for maximal applicability of the model in current care on pregnancy after CS. As both expert opinions and literature were part of the predictor selection process, we developed a model that is actual appropriate and easy to use. Therefore, unlike other models, we included only factors that can be known prior to delivery. For instance, models have been described that include birth weight, which is only known postpartum, and we chose not to include intrapartum factors like cervical examination since we aimed for decision-making in the early third trimester. Several limitations in this study should also be addressed. Firstly, though for most variables we have collected a complete dataset, we had a significant amount of missing values mainly for BMI and EFW because these data were not available in the charts. Since the amount of missing data for BMI was merely resulting from a few particular hospitals, these missing values were considered to be missing at random and we consider the effect on model development and performance to be minor. This was confirmed by comparing performance parameters calculated with complete case analysis to the imputed dataset. Another drawback of our study is that we were not yet able to assess the next steps within prognostic modelling: determining external validity, usefulness and clinical impact of the model. Therefore, though model development was thorough and based on contemporary guidelines within prognostic modelling, our current results should be interpreted with caution.

\section{Interpretation}

This is the first model for prediction of successful intended VBAC that includes EFW. The other selected predictor variables have been described other studies.5,6,8 Also, the discriminative capacity of our model was comparable to those reported in other studies. ${ }^{5,8}$ An exception to all published models might be the model of Naji et al. ${ }^{23}$ which showed promising results with a model incorporating ultrasound evaluation of the CS-scar in the lower uterus segment. Also a recent published study of Verhoeven et al. ${ }^{24}$ showed, as the study of Smith et al. ${ }^{7}$, that maternal height may be an independent predictor for emergency CS. Hence our predictor selection methods did not lead to inclusion of these variables at the moment of model development. Future studies should evaluate whether inclusion of CSscar variables and maternal height increase accuracy of VBAC prediction. Concerning other VBAC prediction studies, it was stated in a systematic review of Eden et al. ${ }^{8}$ that most models have the lack to predict failure of intended VBAC. Our model achieved the highest accuracy for predicted probabilities in the higher probability ranges as well, though overall performance parameters are good. However unlike other tools, our model is targeted on a 
Western European population and prediction in the early third trimester, incorporating both factors that are known prior to pregnancy and factors that occur during pregnancy. Though our dataset was smaller than some other prediction models ${ }^{9,10}$ we achieved an adequate sample size for model development and by preselecting potential predictors using other studies reporting on VBAC prediction, it is likely that our results are generalizable. Therefore, this new prediction model may provide a more appropriate and applicable alternative for countries with a West-European population than existing models.

Concurrently with the globally rising primary CS rates ${ }^{25}$, increasing numbers of women are pregnant with a history of CS. The need for healthcare interventions in order to enhance decision-making on mode of birth after CS has been addressed in several studies. ${ }^{5}$ We think that a prediction model can be applied to enhance shared decision making and in order to place risks of morbidity of intended VBAC into context. Usefulness of such a model is likely as the prediction model could contribute to more unbiased and accurate counselling; and might lead to a reduction of emergency CSs. In order to test this hypothesis, we currently carry out a prospective study in which we evaluate the developed prediction model through application in order to determine external validity, usefulness and clinical impact. To the knowledge of the authors, no clinical impact studies have been published of other existing models on this topic. However, we think that this is essential since model application might lead to different birth preferences resulting in a different selection of women undergoing intended VBAC. This selection might alter performance parameters of the model. ${ }^{26}$ Consequently, models should be evaluated through application before implementation into clinical practice. The prediction model is tested in a prospective controlled setting in twelve hospitals in The Netherlands. In six intervention hospitals we apply the model as a part of a patient decision aid (PtDA) on mode of delivery after previous CS and in six control hospitals we evaluate the prediction model without actually applying the model. For the intervention hospitals, the model is adapted into an easy to use calculator that is used in the early third trimester. Pretesting of this prediction model and PtDA in 25 women within the target group showed that the majority of the women judged the prediction model to be relevant for decision-making.

\section{Conclusion}

We developed an appropriate Western-European population based prediction model that is aimed for counselling on mode of birth after CS for term deliveries. The model holds promise as a tool that personalises decision-making on mode of birth after CS and could be applied to place potential risks of intended VBAC into context. 


\section{References}

1. Bujold E. Evaluating professional society guidelines on vaginal birth after cesarean. Semin Perinatol 2010; 34(5): 314-7.

2. McMahon MJ, Luther ER, Bowes WA, Jr., Olshan AF. Comparison of a trial of labor with an elective second cesarean section. NEngl J Med 1996; 335(10): 689-95.

3. Shorten A, Shorten B. The importance of mode of birth after previous cesarean: success, satisfaction, and postnatal health. J Midwifery Womens Health 2012; 57(2): 126-32.

4. Shorten A, Shorten B, Keogh J, West S, Morris J. Making choices for childbirth: a randomized controlled trial of a decision-aid for informed birth after cesarean. Birth 2005; 32(4): 252-61.

5. Guise JM, Eden K, Emeis C, et al. Vaginal birth after cesarean: new insights. Evid Rep Technol Assess (Full Rep) 2010; (191): 1-397.

6. Grobman WA. Rates and prediction of successful vaginal birth after cesarean. Semin Perinatol 2010; 34(4): 244-8.

7. Smith GC, White IR, Pell JP, Dobbie R. Predicting cesarean section and uterine rupture among women attempting vaginal birth after prior cesarean section. PLoS Med 2005; 2(9): e252.

8. Eden $\mathrm{KB}, \mathrm{McDonagh} \mathrm{M}$, Denman MA, et al. New insights on vaginal birth after cesarean: can it be predicted? Obstet Gynecol 2010; 116(4): 967-81.

9. Grobman WA, Lai Y, Landon MB, et al. Development of a nomogram for prediction of vaginal birth after cesarean delivery. Obstet Gynecol 2007; 109(4): 806-12.

10. Grobman WA, Lai Y, Landon MB, et al. Does information available at admission for delivery improve prediction of vaginal birth after cesarean? Am J Perinatol 2009; 26(10): 693-701.

11. Schoorel EN, Melman S, van Kuijk SM, et al. Predicting successful intended vaginal delivery after previous caesarean section: external validation of two predictive models in a Dutch nationwide registration-based cohort with a high intended vaginal delivery rate. BJOG 2014; 121(7): 840-7; discussion 7.

12. Steyerberg EW. Clinical Prediction Models. New York NS.

13. Harrell FJ. Regression Modeling Strategies. New York NS.

14. Steyerberg EW, Moons KG, van der Windt DA, et al. Prognosis Research Strategy (PROGRESS) 3: prognostic model research. PLoS Med 2013; 10(2): e1001381.

15. National Institutes of Health Consensus Development Conference Statement vaginal birth after cesarean: new insights March 8-10, 2010. Semin Perinatol 2010; 34(5): 351-65.

16. Snijders RJ, Nicolaides KH. Fetal biometry at 14-40 weeks' gestation. Ultrasound Obstet Gynecol 1994; 4(1): 3448.

17. Yudkin PL, Aboualfa M, Eyre JA, Redman CW, Wilkinson AR. New birthweight and head circumference centiles for gestational ages 24 to 42 weeks. Early Hum Dev 1987; 15(1): 45-52.

18. Hadlock FP, Harrist RB, Sharman RS, Deter RL, Park SK. Estimation of fetal weight with the use of head, body, and femur measurements--a prospective study. Am J Obstet Gynecol 1985; 151(3): 333-7.

19. Peduzzi P, Concato J, Kemper E, Holford TR, Feinstein AR. A simulation study of the number of events per variable in logistic regression analysis. J Clin Epidemiol 1996; 49(12): 1373-9.

20. Kwee A, Bots ML, Visser GH, Bruinse HW. Obstetric management and outcome of pregnancy in women with a history of caesarean section in the Netherlands. Eur J Obstet Gynecol Reprod Biol 2007; 132(2): 171-6.

21. Donders AR, van der Heijden GJ, Stijnen T, Moons KG. Review: a gentle introduction to imputation of missing values. J Clin Epidemiol 2006; 59(10): 1087-91.

22. Rubin DB. Inference and missing data. Biometrika. 1976;63(3):581-92.

23. Naji O, Wynants L, Smith A, et al. Predicting successful vaginal birth after Cesarean section using a model based on Cesarean scar features examined by transvaginal sonography. Ultrasound Obstet Gynecol 2013; 41(6): $672-8$

24. Verhoeven CJ, van Uytrecht CT, Porath MM, Mol BW. Risk factors for cesarean delivery following labor induction in multiparous women. J Pregnancy 2013; 2013: 820892.

25. Brennan DJ, Murphy M, Robson MS, O'Herlihy C. The singleton, cephalic, nulliparous woman after 36 weeks of gestation: contribution to overall cesarean delivery rates. Obstet Gynecol 2011; 117(2 Pt 1): 273-9.

26. Van Kuijk SM, Sep SJ, Nelemans PJ, Smits LJ. How long do preconception risk prediction models hold? Influence of selective fertility on model performance. Paediatr Perinatal Epidemiol 2010; 24(6): 602-7. 


\section{Chapter 7}

\section{Probability of vaginal birth after caesarean:}

do not ask the obstetrician

ENC Schoorel, DRM Hünen, SMJ van Kuijk, BCC Augustijn, A Kwee, BWJ Mol, JG Nijhuis, LJM Smits, RPMG Hermens, HCJ Scheepers 


\section{Abstract}

\section{Background}

There is an increasing number of women with a prior caesarean section (CS) who deliver by elective repeat CS (ERCS) instead of intended vaginal birth after caesarean (VBAC). The probability of successful VBAC plays a central role within risk estimation for mode of birth. We aimed to determine predictive performance of clinicians for successful VBAC compared to a prediction model and used actual clinical outcomes as a benchmark.

\section{Methods}

Clinical vignettes were constructed from actual patients with known outcomes of intended VBAC (successful or failed). We randomly drew 450 clinical cases in order to create vignettes. For each vignette, the probability of successful VBAC was estimated both by a clinician and prediction model, and compared to the actual outcome. Discriminative performance was assessed by obtaining the areas under the curve (AUC) of the receiver operator characteristic; predictive performance was assessed by calibration per-risk-quantile and Hosmer-Lemeshow (H-L) statistics.

\section{Findings}

Four-hundred-and-six vignettes were completed. For clinicians, the AUC was 0.60 (CI 95\% 0.54-0.67) while the AUC for the prediction model was 0.70 (CI 95\% 0.64-0.76) (p-value for difference $\mathrm{P}<0.003)$. Calibration-per-risk-quantile showed that clinicians generally underestimated the probability of successful intended VBAC below 70.0\%. Calibration-perrisk-quantile for the prediction model was good in all probability ranges. The corresponding H-L statistic confirmed lack of fit for clinicians $(P=0.024)$, no evidence was found for lack of fit for the prediction model $(P=0.42)$.

\section{Interpretation}

Compared to the model, clinicians performed poorly with regard to prediction of successful intended VBAC whilst the majority counsels with a self-derived probability. Hence, in comparison to clinical judgement, the use of the VBAC prediction model can be beneficial. 


\section{Introduction}

Worldwide rising caesarean section (CS) rates are a major burden on healthcare. Reported contemporary CS rates are as high as 25\% in England and 33\% in The United States (US) ${ }^{1}$, with a range of $17 \%$ in The Netherlands to $44 \%$ in Mexico. ${ }^{2,3}$ It is remarkable that the largest increase in CSs has been observed amongst nulliparous women with a singleton in vertex position who deliver at term. ${ }^{4}$ With these rising numbers, the debate on safety and practice of childbirth after CS becomes more pressing. It is concerning that increasing numbers of women with a prior CS deliver by elective repeat caesarean section (ERCS) instead of intended vaginal birth after caesarean (VBAC) (i.e. trial of labour after caesarean). ${ }^{5}$ Though some hospitals entirely banned VBAC because of fear for litigation ${ }^{6,7}$, most experts do not consider ERCS to be superior to intended VBAC. In fact, contemporary guidelines on pregnancy and childbirth after CS state that women should be counselled in order to empower them to choose between the birth options. ${ }^{8-11}$ This difficult decision-making process is influenced by many factors. ${ }^{12}$ Amongst these factors, the probability of successful intended VBAC (i.e. actual VBAC) plays a central role. Reports have linked a low probability of success to a high risk of major foeto-maternal morbidity. ${ }^{13-15}$ Generally, reported successful VBAC-rates vary between $49 \%$ and $87 \%{ }^{16}$ However, it is evident that individual probabilities vary due to demographic factors, obstetrical history and events occurring during pregnancy. ${ }^{17}$ Many attempts have been made to develop scoring tools that should support the decision-making process by individual risk estimation of successful intended $\mathrm{VBAC}^{16,18}$ and the majority achieves reasonable predictive performance. ${ }^{18}$ To what extent these models could contribute to current care is not clear as data that compares performance of these tools to clinical judgement are not available. Hence, the aim of this study is to determine clinicians' predictive performance for successful intended VBAC in terms of calibration and discrimination and to compare this to a recently developed prediction model.

\section{Methods}

\section{Study design}

This study was designed as a vignette study with vignettes constructed from a retrospective cohort of real patients with known outcomes of intended VBAC (successful or failed). Vignettes were regarded as actual clinical situations. The main outcome measures of this study are performance parameters of clinical judgement and the prediction model in terms of discriminative capacity and calibration. 


\section{Setting}

This study was carried out within the Dutch research consortium for Women's health. Patient data for the construction of the vignettes were derived from a nationwide registration-based cohort study amongst women with a prior CS who delivered in 2010. Data were collected in 17 independent Dutch hospitals, representative for geographical regions in The Netherlands. Participating hospitals consisted of university teaching hospitals $(\mathrm{n}=5)$, non-university teaching hospitals $(\mathrm{n}=7)$ and non-university non-teaching hospitals $(\mathrm{n}=5)$.

After construction of the vignettes, gynaecologists, residents and midwives were approached for participation through email via the Dutch research consortium for Women's health and during a national conference of the Dutch Society of Gynaecology and Obstetrics (NVOG).

\section{Population}

The cohort used for the construction of the vignettes and the prediction model consists of women who were consecutively included according to the hospitals birth registers. We included women with one prior low-transverse CS and a singleton pregnancy in vertex position. Exclusion criteria were breech presentation or an intrauterine foetal demise or a contraindication for intended VBAC. A contraindication for intended VBAC was defined as placenta previa or a relevant uterine scar with extension into the fundus. For this study, we selected only women from the database who had an intended VBAC.

\section{Sample size considerations}

The sample size calculation was based on a difference in discriminative capacity between the clinicians and the prediction model. A difference of $10 \%$ in the areas under the curve (AUC) of the receiver operator characteristics (ROC) was considered clinically relevant. Therefore, with an alpha of 0.05 and a beta of 0.20 , the estimated required sample size was 400 vignettes.

\section{Vignette derivation}

Patient characteristics included in the vignettes were selected based on prior research findings, an evidence report on $\mathrm{VBAC}^{16}$, expert opinions and availability in the database. The selected variables were: third trimester estimated foetal weight (EFW) $\geq$ percentile ( $p$ ) 90 (yes/no), EFW $\leq$ p10 (yes/no), prior CS due to non-progressive labour (yes/no), one or more previous vaginal deliveries (yes/no), induction of labour in current pregnancy (yes $/ \mathrm{no}$ ), BMI $\left(\mathrm{kg} / \mathrm{m}^{2}\right)$, ethnicity, maternal age (years), hypertension (HT) (yes/no), diabetes mellitus (DM) (yes/no) and the occurrence of pre-eclampsia (PE) or HELLP syndrome during current pregnancy. A prior CS due to non-progressive labour was defined as a failure 
of dilation or descend in the first or second stage of labour. Pre-pregnancy body mass index $\left(\mathrm{kg} / \mathrm{m}^{2}\right)$ (BMI) was defined as BMI prior to, or in the first trimester of the current pregnancy. DM and HT could be either pre-existent or gestational induced. Before these data were copied to standardized clinical vignettes, missing data were imputed since omission of incomplete cases can result in loss of precision and may bias the results. ${ }^{19,} 20$ For imputation, all quantitative baseline characteristics were used for estimation of the missing values, the number of imputations were set to five. Subsequently for vignette derivation, 450 cases were randomly drawn with replacement from the database.

\section{Survey}

We aimed to obtain a sample of clinicians, representative of providers working within obstetrics and gynaecology in The Netherlands, by using national networks (see setting) for recruitment. Participants were approached face-to-face on a conference or through massemail. Non-responders received a reminder email. No compensation was provided for participation. Participants completed one to three vignettes where they were asked to predict a probability of successful intended VBAC ranging from $0 \%$ and $100 \%$. In order to obtain insight in possible participation bias, we collected data on experience and views of the participants on counselling on mode of birth after caesarean. Questions were directed at the number of years of experience as registered obstetrician, the frequency of counselling and the place of the probability of successful intended VBAC within counselling practice. Participants were subdivided in obstetricians, and non-obstetricians (residents, midwives).

\section{Prediction model}

Our prediction model was developed for third trimester counselling amongst 515 women with a history of one CS. Potential predictors for successful intended VBAC were chosen based on literature and expert opinion; the model was internally validated using bootstrapping techniques. The development, interval validation and performance of this model is described in more detail elsewhere. ${ }^{21}$ The final predictive equation is as follows: $\mathrm{P}_{\text {(success) }}=(1 /(1+\exp (-(1.647+0.371 *$ Caucasian $-0.032 *$ pre-pregnancy body mass index $0.537 *$ Previous non progressive labour $+1.045 *$ Previous vaginal delivery $-0.515 *$ Induction of labour $-0 \cdot 487 *$ third trimester estimated foetal weight $\geq \mathrm{p} 90)))) * 100 \%$. Definitions match those of the clinical vignettes.

\section{Statistical analyses}

For each clinical vignette, the probability of successful intended VBAC was calculated with the prediction model, and assessed by one or more clinicians. Subsequently, parameters of discriminative and predictive performance were obtained for both the prediction model and for the clinicians. Discriminative performance was assessed with a ROC-analysis by 
obtaining the corresponding AUC. The ROC was obtained by plotting sensitivity against 1specificity. Within this analysis, sensitivity was defined as the fraction of successful intended VBAC that was predicted correctly per corresponding cut-off value. Specificity was defined as the fraction of unsuccessful intended VBAC that was correctly predicted per corresponding cut-off value. The AUC reflects the ability of the prediction tool (i.e. model or clinicians) to discriminate between women who have a high probability of successful intended VBAC and women who have a low probability of successful intended VBAC. Predictive performance, the agreement between predicted and observed values, was assessed per risk quantile. Calibration was assessed by visually inspecting the agreement between observed and predicted frequencies in the calibration plots. Goodness-of-fit to the ideal calibration curve was quantified with the Hosmer-Lemeshow (H-L) statistic. A high H$\mathrm{L}$ statistic is related to a low p-value and indicates lack of fit. Additionally, a subgroup analyses was performed concerning differences in predictive ability of obstetricians and non-obstetricians (residents/midwives). We evaluated the difference in AUC's using the method of DeLong, extended for non-correlated ROC-curves. Additional sensitivity analysis was performed to determine whether the presence of double vignettes biased results. Estimated probabilities of VBAC for one clinical case were combined to recalculate the outcome measures.

All statistical analyses were performed using SPSS (version 20.0) and R (version 2.12.2; http://www.r-project.org).

\section{Results}

\section{Study population}

The patient cohort comprised 515 women with a prior CS who had an intended VBAC of whom $371(72.0 \%)$ actually delivered vaginally. Baseline characteristics of the cohort are summarized in Table 7.1.

Mean age was 32.2 years (standard deviation (SD) 5.0). 405 (81\%) women were of Caucasian ethnicity. In this cohort, mean BMI was 25.3 (SD 5.7). In total, 127 (24.7\%) women had a previous vaginal delivery, 201 (39\%) had a prior CS because of nonprogressive labour. In the current pregnancy, 132 (25.6\%) women were induced, nine (1.7\%) women had PE or HELLP, 13 (2.5\%) had DM and 37 (7.2\%) had HT. EFW was lower or equal to P10 in $26(8.3 \%)$ cases and higher or equal to P90 in 11 (3.5\%) cases. 
Table 7.1 Study cohort. Baseline characteristics of study cohort of women with a trial of labour after caesarean used for derivation of the clinical vignettes.

\begin{tabular}{|c|c|c|}
\hline Characteristics & $\begin{array}{c}\text { Missings } \\
\text { N }(\%)\end{array}$ & $\begin{array}{l}\text { Cohort } \\
(n=515)\end{array}$ \\
\hline Maternal age (years, mean $\pm \mathrm{SD}$ ) & $2(0 \cdot 0)$ & $32 \cdot 2 \pm 5$ \\
\hline \multicolumn{3}{|l|}{ Ethnicity } \\
\hline Caucasian & $15(3 \cdot 0)$ & $405(81 \cdot 0)$ \\
\hline Mediterranean & & $37(7 \cdot 4)$ \\
\hline African & & $24(4 \cdot 8)$ \\
\hline Indo-Surinamese & & $12(2 \cdot 4)$ \\
\hline Asian & & $7(1 \cdot 4)$ \\
\hline Other & & $15(3 \cdot 0)$ \\
\hline $\mathrm{BMI}\left(\mathrm{kg} / \mathrm{m}^{2}\right.$, mean $\left.\pm \mathrm{SD}\right)$ & $124(24 \cdot 0)$ & $25 \cdot 3 \pm 5 \cdot 7$ \\
\hline Previous CS due to non progressive labour, n (\%) & $0(0 \cdot 0)$ & $201(39)$ \\
\hline Previous vaginal delivery, n (\%) & $0(0 \cdot 0)$ & $127(24 \cdot 7)$ \\
\hline PE/HELLP, n (\%) & $0(0 \cdot 0)$ & $9(1 \cdot 7)$ \\
\hline Diabetes Mellitus, $\mathrm{n}(\%)$ & $0(0 \cdot 0)$ & $13(2 \cdot 5)$ \\
\hline Hypertension, n (\%) & $0(0 \cdot 0)$ & $37(7 \cdot 2)$ \\
\hline \multicolumn{3}{|l|}{ EFW, n $(\%)$} \\
\hline $\mathrm{P} \leq 10$ & $201(39 \cdot 0)$ & $26(8 \cdot 3)$ \\
\hline $\mathrm{P} \geq 90$ & $201(39 \cdot 0)$ & $11(3 \cdot 5)$ \\
\hline Induction of labour, $\mathrm{n}(\%)$ & $0(0 \cdot 0)$ & $132(25 \cdot 6)$ \\
\hline
\end{tabular}

SD: standard deviation, BMI: body mass index, CS: caesarean section, PE: Preeclampsia, HELLP syndrome: haemolysis, elevated liver-enzymes, low platelets, P: percentile.

\section{Participants}

In total, 406 vignettes were completed of which $255(62 \cdot 8 \%)$ by obstetricians and 147 $(36.2 \%)$ by non-obstetricians (residents and midwives). Characteristics of the participants are summarized in Table 7.2.

Table 7.2 Participants. Characteristics of the study participants, i.e. clinicians.

\begin{tabular}{lccc}
\hline $\begin{array}{l}\text { Characteristics } \\
\mathbf{n}=\mathbf{4 0 6 *}\end{array}$ & $\begin{array}{c}\text { Missings } \\
\mathbf{n} / \mathbf{n}\end{array}$ & $\begin{array}{c}\text { Obstetrician } \\
\mathbf{n}=\mathbf{2 5 5}\end{array}$ & $\begin{array}{c}\text { Non-obstetrician } \\
\mathbf{n}=\mathbf{1 4 7}\end{array}$ \\
\hline Sex, $\mathrm{n}(\%)$ & $0 / 0$ & $111(43 \cdot 5)$ & $15(10 \cdot 2)$ \\
Male & & $144(56 \cdot 5)$ & $132(89 \cdot 8)$ \\
Female & & $45 \cdot 1 \pm 7 \cdot 8$ & $31 \cdot 0 \pm 5 \cdot 7$ \\
Age, (years, mean \pm SD) & $3 / 0$ & & \\
Setting, n (\%) & $0 / 2$ & $54(21 \cdot 2)$ & $59(40 \cdot 7)$ \\
Academic teaching hospital & & $138(54 \cdot 1)$ & $81(55 \cdot 9)$ \\
Non academic teaching hospital & & $63(24 \cdot 7)$ & $1(0 \cdot 7)$ \\
Non academic non-teaching hospital & & $0(0 \cdot 0)$ & $4(2 \cdot 8)$ \\
Midwivery practice & $0(0 \cdot 0)$ & & \\
Experience, $\mathrm{n}(\%)$ & & $72(28 \cdot 2)$ & $\mathrm{N} / \mathrm{A}$ \\
$<5$ years & & $85(33 \cdot 3)$ & $\mathrm{N} / \mathrm{A}$ \\
5-10 years & & $98(38 \cdot 4)$ & N/A \\
$>10$ years & & & \\
\hline
\end{tabular}

* Of four participants it was unkown whether they were obstetrician or non-obstetrician. SD: standard deviation ; N/A not applicable 
In Table 7.3, data with regard to the views and experiences of the participants with risk counselling on mode of birth after CS are summarized. A majority of the participants counselled on mode of birth more than 20 times per year, and a majority of the participants always provided a probability of successful intended VBAC during every counselling. Obstetricians provided in $57.0 \%$ a self-estimated probability of successful intended VBAC, while residents and midwives more often provided the probability that was stated in the Dutch guidelines.

Table 7.3 Risk-counselling. Overview of experience and views of the participants on risk counselling on mode of birth after caesarean.

\begin{tabular}{|c|c|c|c|}
\hline $\begin{array}{l}\text { Questions } \\
\mathrm{n}=406^{*}\end{array}$ & $\begin{array}{l}\text { Missings } \\
\mathrm{n} / \mathrm{n}\end{array}$ & $\begin{array}{c}\text { Obstetrician } \\
\mathrm{n}=255\end{array}$ & $\begin{array}{c}\text { Non-obstetrician } \\
\mathrm{n}=147\end{array}$ \\
\hline How frequently do you counsel on mode of birth after CS? & $4 / 20$ & & \\
\hline$<5$ times/year, $\mathrm{n}(\%)$ & & $36(14 \cdot 3)$ & $23(18 \cdot 1)$ \\
\hline 5-20 times/year, n (\%) & & $66(26 \cdot 3)$ & $52(40 \cdot 9)$ \\
\hline$>20$ times/year, n (\%) & & $149(59 \cdot 4)$ & $52(40 \cdot 9)$ \\
\hline $\begin{array}{l}\text { How often do you provide a probability of successful } \\
\text { intended VBAC during counselling? }\end{array}$ & $7 / 11$ & & \\
\hline Always, n (\%) & & $151(60 \cdot 9)$ & $63(46 \cdot 3)$ \\
\hline Sometimes, $\mathrm{n}(\%)$ & & $66(26 \cdot 6)$ & $49(36 \cdot 0)$ \\
\hline Never, n (\%) & & $31(12 \cdot 5)$ & $24(17 \cdot 6)$ \\
\hline $\begin{array}{l}\text { What probability of successful intended VBAC do you } \\
\text { generally provide during counselling? }\end{array}$ & $41 / 41$ & & \\
\hline A self-estimated individual probability, n (\%) & & $122(57 \cdot 0)$ & $34(32 \cdot 1)$ \\
\hline Conform Dutch guidelines (70-75\%), n (\%) & & $48(22 \cdot 4)$ & $53(50 \cdot 0)$ \\
\hline Other, $\mathrm{n}(\%)$ & & $44(20 \cdot 6)$ & $19(17 \cdot 9)$ \\
\hline
\end{tabular}

* Of four participants it was unknown whether they were obstetrician or non-obstetrician. CS: caesarean section; ERCS: elective repeat caesarean section; VBAC: vaginal birth after caesarean

\section{Main results}

The discriminative capacities of clinicians and the prediction model are shown in Figure 7.1. The ROC of the clinicians has an AUC of 0.60 (CI 95\% 0.54-0.67), indicating poor discriminative capacity. The ROC curve of the prediction model has an AUC of 0.70 (CI $95 \% 0.64-0.76)$ which indicates fair discriminative capacity. The difference between the AUC's of the predictive ability of clinicians and the model is $10.0 \%(P<0.003)$.

Additional comparison of the AUC's of obstetricians (0.61) to non-obstetricians (0.60) showed no meaningful difference $(P=0.8)$. In Figure 7.2, calibration-per-risk-quantile for predictive performance of clinicians is shown. Clinicians generally underestimated the probability of successful intended VBAC below $70.0 \%$. Above $70.0 \%$, calibration was fair. The corresponding H-L statistic however, shows that accuracy was poor $(P=0.024)$. 


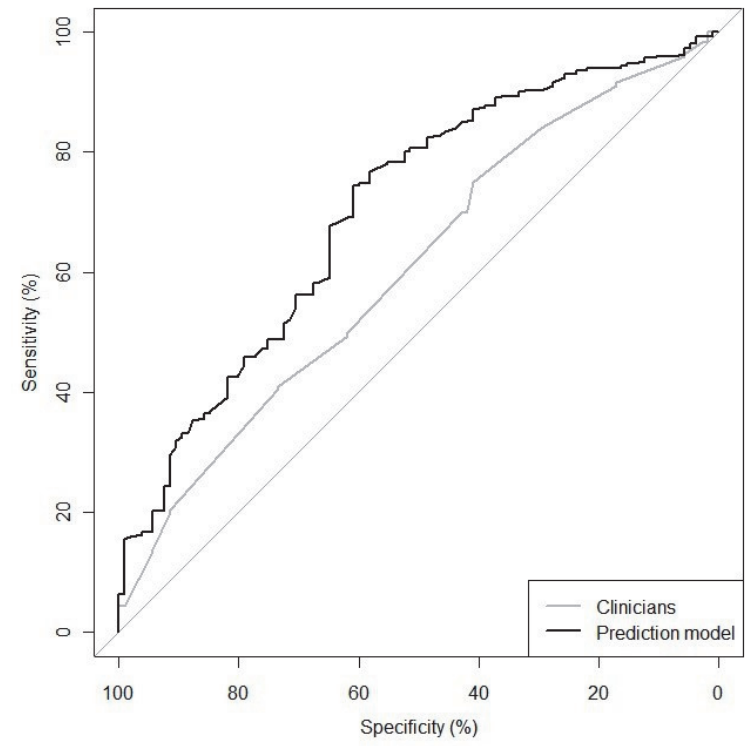

Figure 7.1 Receiver operating characteristics. Receiver operating characteristics of clinicians (AUC 0.60; 95\% CI: 0.54-0.67), and the prediction model (AUC 0.70; 95\% CI: 0.64-0.76).

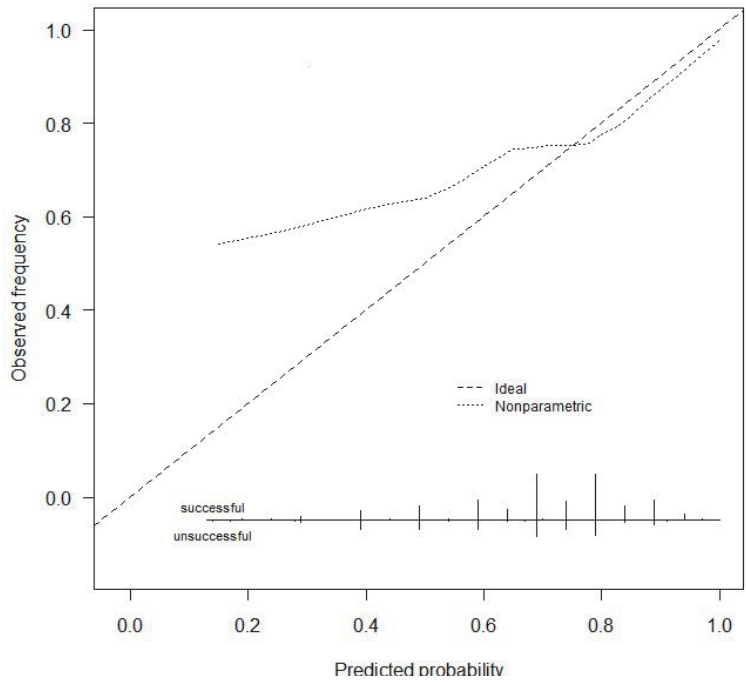

Figure 7.2 Calibration clinicians. Calibration plot with the observed frequency of a successful trial of labour after caesarean section in the study cohort by probabilities estimated by the clinicians. 
Figure 7.3 shows calibration-per-risk-quantile for the prediction model. The prediction model was well calibrated over the whole range of probabilities. The corresponding H-L statistic shows a p-value of 0.42 , which indicates a good fit of the model. Vignettes were multiple drawn with a maximum of three times; sensitivity analysis revealed that the presence of double or triple vignettes did not lead to radically different results or conclusions.

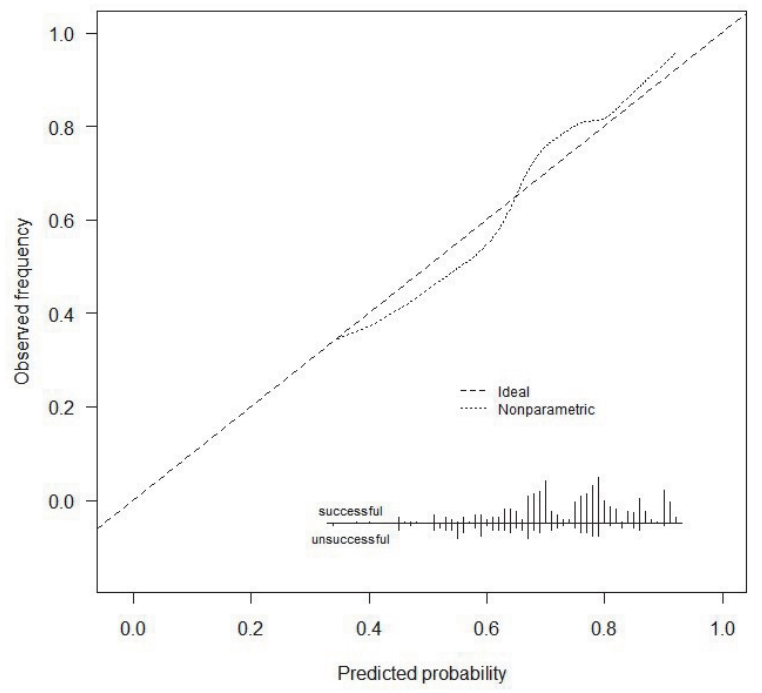

Figure 7.3 Calibration prediction model. Calibration plot with the observed frequency of a successful trial of labour after caesarean section in the study cohort by probabilities estimated with the prediction model.

\section{Discussion}

Both obstetricians and residents/midwifes had experience with counselling on mode of delivery after CS. However, our results show that clinicians do not perform well with regard to prediction of successful intended VBAC. Both accuracy and discrimination between women with a high probability and a low probability was poor. In general, clinicians underestimate the actual probability of success, this may lead to more ERCS prone counselling. Subgroup analysis revealed no meaningful difference in performance between obstetricians and non-obstetricians, while obstetricians clearly counsel more often with a self-perceived probability instead of the risk-numbers of contemporary guidelines. This study also shows that the use of the developed prediction model can be beneficial as with the prediction model a higher predictive and discriminative performance was achieved. 
The findings in this paper are consistent with the conclusions of Grove and colleagues ${ }^{22}$, who performed a systematic literature review on studies that compare 'statistical' prediction and 'clinical' prediction. In this review it was concluded that in general, prediction models are $10 \%$ more accurate than clinical judgement. ${ }^{22}$ The prediction model that was used as a reference in this study performs similar to other models for prediction of successful intended VBAC. ${ }^{18}$ The majority of women get a score with the prediction model of $70 \%$ or higher. Studies showed that for this group, risks on severe foeto-maternal morbidity for intended VBAC are similar to those of ERCS. ${ }^{14,} 15$ Therefore, women with an estimated chance of actual VBAC of $>70 \%$ could proceed with intended VBAC, preventing morbidity for future pregnancies that results from each extra CS-scar. ${ }^{23}$ Also, this could reduce healthcare costs as failed intended VBAC is more expensive in comparison to ERCS and successful intended VBAC respectively. ${ }^{24}$ However, before a prediction model is incorporated into clinical practice, we recommend a thorough prospective evaluation through application.

To the knowledge of the authors, this is the first study that estimates to what extent clinicians use the probability of successful intended VBAC within counselling and whether they are able to estimate a reliable chance of success based on knowledge i.e. 'skilled intuition'. A strong point of this study is that the design enables us to compare predictive performance of clinicians to a reference, as provided with the prediction model and the actual outcomes. This approach is in concurrence with a recent review of Bachmann and colleagues on vignette studies who stated these studies should compare outcomes to a benchmark. ${ }^{25}$ Therefore, we were able to determine 'correctness' of the prediction very accurately. However, the ideal design for this study is a prospective study with 'actual' patients instead of vignettes in a fixed format. The main concern is that it is questionable whether professionals in reality indeed behave and judge as they do in the hypothetical context of a clinical vignette. ${ }^{25}$ Furthermore, the predefining of variables might have resulted in loss of information relevant to individual clinicians and could therefore have induced a loss of precision of our results. Another drawback of our design was related to the retrospective nature of our study as well, as we were not able to obtain all patient data. Therefore, we were required to impute missing values up to $39.0 \%$ of all data regarding EFW and $24.0 \%$ for BMI which may have impaired precision of predicted probabilities of VBAC. Further, though we obtained a representative sample of clinicians for the Dutch setting, the additional 'gain' of using a prediction model for individual risk estimation might be varying across countries based on clinical training and local practices. 
In conclusion, our results show that clinicians perform poorly with regard to the estimation of the probability of successful VBAC. Our results are in favour of the prediction model since it is more reliable than clinical prediction and is easy to use. Implementation of the prediction model in current care might lead to a more standardized care. Also, as clinicians generally underestimated the VBAC success rates, the usage of a prediction model might lead to a different, more VBAC prone, mind-set within counselling in both clinicians and patients.

\section{References}

1. Martin JA, Hamilton BE, Ventura SJ, et al. Births: final data for 2009. National vital statistics reports : from the Centers for Disease Control and Prevention, National Center for Health Statistics, National Vital Statistics System 2011; 60(1): 1-70.

2. Nederland SPR. Perinatale Zorg in Nederland 2010. Utrecht: Stichting Perinatale Registratie Nederland, 2013

3. Boyle A, Reddy UM. Epidemiology of cesarean delivery: the scope of the problem. Semin Perinatol 2012; 36(5): 308-14.

4. Brennan DJ, Murphy M, Robson MS, O'Herlihy C. The singleton, cephalic, nulliparous woman after 36 weeks of gestation: contribution to overall cesarean delivery rates. Obstet Gynecol 2011; 117(2 Pt 1): 273-9.

5. National Institutes of Health Consensus Development conference statement: vaginal birth after cesarean: new insights March 8-10, 2010. Obstet Gynecol 2010; 115(6): 1279-95.

6. Barger MK, Dunn JT, Bearman S, Delain M, Gates E. A survey of access to trial of labor in California hospitals in 2012. BMC Pregnancy Childbirth 2013; 13(1): 83.

7. Roberts RG, Deutchman M, King VJ, Fryer GE, Miyoshi TJ. Changing policies on vaginal birth after cesarean: impact on access. Birth 2007; 34(4): 316-22.

8. American congress of Obstetricians and Gynaecologists. Vaginal birth after previous cesarean delivery. ACOG Practice Bulletin 2010; (no. 115).

9. The society of Obstetricians and Gynaecologists of Canada. Guidelines for Vaginal Birth After Previous Caesarean Birth. J Obstet Gynaecol Can 2005; (155): 89: 319-31.

10. Dutch organisation of Obstetrics and Gynaecology. Pregnancy and Childbirth after previous caesarean section. 2010.

11. Royal college of Obstetricians and Gynaecologists. Birth after previous caesarean birth. Green-top Guideline 2007; No. 45.

12. Kaimal AJ, Kuppermann M. Understanding risk, patient and provider preferences, and obstetrical decision making: approach to delivery after cesarean. Semin Perinatol 2010; 34(5): 331-6.

13. McMahon MJ, Luther ER, Bowes WA, Jr., Olshan AF. Comparison of a trial of labor with an elective second cesarean section. N Engl J Med 1996; 335(10): 689-95.

14. Grobman WA, Lai Y, Landon MB, et al. Can a prediction model for vaginal birth after cesarean also predict the probability of morbidity related to a trial of labor? Am J Obstet Gynecol 2009; 200(1): 56 e1-6.

15. Chaillet N, Bujold E, Dube E, Grobman WA. Validation of a prediction model for predicting the probability of morbidity related to a trial of labour in quebec. JOGC 2012; 34(9): 820-5.

16. Guise JM, Eden K, Emeis C, et al. Vaginal birth after cesarean: new insights. Evid Rep Technol Assess (Full Rep) 2010; (191): 1-397.

17. Grobman WA. Rates and prediction of successful vaginal birth after cesarean. Semin Perinatol 2010; 34(4): 244-8.

18. Eden KB, McDonagh M, Denman MA, et al. New insights on vaginal birth after cesarean: can it be predicted? Obstet Gynecol 2010; 116(4): 967-81.

19. Harrell F. Regression Modeling Strategies. New York: NY: Springer; 2001.

20. Steyerberg E. Clinical Prediction Models. New York: NY: Springer; ; 2009. 
21. Schoorel E, van Kuijk S, Melman S, et al. Vaginal birth after a caesarean section: the development of a Western European population-based prediction model for deliveries at term. BJOG 2014; 121(2): 194-201.

22. Grove WM, Zald DH, Lebow BS, Snitz BE, Nelson C. Clinical versus mechanical prediction: a metaanalysis. Psychol Assess 2000; 12(1): 19-30.

23. Silver RM, Landon MB, Rouse DJ, et al. Maternal morbidity associated with multiple repeat cesarean deliveries. Obstet Gynecol 2006; 107(6): 1226-32.

24. Macario A, El-Sayed YY, Druzin ML. Cost-effectiveness of a trial of labor after previous cesarean delivery depends on the a priori chance of success. Clin Obstet Gynecol 2004; 47(2): 378-85.

25. Bachmann LM, Muhleisen A, Bock A, ter Riet G, Held U, Kessels AG. Vignette studies of medical choice and judgement to study caregivers' medical decision behaviour: systematic review. BMC Med Res Methodol 2008; 8: 50 . 


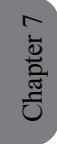




\section{Chapter 8}

\section{Involving women in personalised decision-making}

on mode of delivery after caesarean section: the development and pilot testing of a patient decision aid

ENC Schoorel, E Vankan, HCJ Scheepers, BCC Augustijn, CD Dirksen, M de Koning, SMJ van Kuijk, A Kwee, S Melman, JG Nijhuis, R Aardenburg, K de Boer, THM Hasaart, BWJ Mol, M Nieuwenhuijze, MG van Pampus, J van Roosmalen, FJME Roumen, R de Vries, MGAJ Wouters, T van der Weijden, RPMG Hermens BJOG: An international journal of Obstetrics and Gynaecology 2014;121:202-209 


\section{Abstract}

Objective To develop a patient decision aid (PtDA) for mode of delivery after caesarean section (CS) that integrates personalised prediction of vaginal birth after caesarean (VBAC) with elicitation of patient preferences and evidence-based information.

\section{Design and setting}

A PtDA was developed and pilot tested using the International Patients Decision Aid Standards (IPDAS) criteria.

Obstetric healthcare in the Netherlands.

\section{Population}

A multidisciplinary steering group, an expert panel and 25 future users of the PtDA, i.e. women with a previous CS.

\section{Methods}

Development consisted of a construction phase (definition of scope and purpose, selection of contents, framework and format) and a pilot-testing phase by interview. The process was supervised by a multidisciplinary steering group.

\section{Main outcome measures}

Usability, clarity, relevance.

\section{Results}

The construction phase resulted in a booklet including unbiased balanced information on mode of birth after CS, a preference elicitation exercise and tailored risk information including a prediction model for successful VBAC. During pilot testing, visualisation of risks and clarity formed the main basis for revisions. Pilot testing showed the availability of tailored structured information to be the main factor involving women in decision-making. The PtDA meets 39 out of 50 IPDAS criteria (78\%): 23 out of 23 criteria for content $(100 \%)$ and 16 out of 20 criteria for the development process $(80 \%)$. Criteria for effectiveness $(n=7)$ were not evaluated.

\section{Conclusions}

An evidence-based PtDA was developed, with the probability of successful VBAC and the availability of structured information as key items. It is likely that the PtDA enhances the quality of decision-making on mode of birth after CS. 


\section{Introduction}

Decision-making in pregnancy is particularly complex because women must consider not only their own health but also that of their unborn babies. In the decision on mode of delivery after caesarean section (CS), the importance of involving and supporting pregnant women has been highlighted by various international guidelines. ${ }^{1-4}$ When no contraindications are present, women should be provided with a choice between an elective repeat CS (ERCS) and an intended vaginal birth after caesarean (VBAC) (i.e. a trial of labour). ${ }^{1-4}$ It is recommended that this decision is based on a discussion of the risks, benefits and implications of both options. ${ }^{1-5}$ Mean reported intended VBAC success rates are as high as $72-76 \%,{ }^{6}$ and a successful VBAC is associated with fewer major complications, ${ }^{7}$ a shorter recovery period and higher perceived maternal satisfaction scores. ${ }^{8,9}$ In addition, an intended VBAC is in general more cost-effective than ERCS. ${ }^{10}$ However the drawback of intended VBAC is possible failure leading to an emergency CS, which is associated with a higher risk of major complications including hysterectomy and operative injury, ${ }^{7}$ and lower levels of maternal satisfaction ${ }^{8,9}$ than with either ERCS or successful VBAC. The existence of major international differences in (intended) VBAC rates suggests that choices for mode of delivery after CS do not always reflect women's preferences but may result from nonmedical factors like hospital policy, concerns about medical liability or provider preference. ${ }^{6}$ The question is how guideline recommendations on mode of birth after CS can be implemented in practice. Due to the complex nature of the decision-making process, and because it concerns a preference-sensitive decision, mode of delivery should be discussed within a shared-decision making (SDM) setting. SDM can be enhanced by the use of patient decision aids (PtDA), which provide unbiased information in a clear and comprehensive way. PtDAs have proven to be effective tools for facilitating communication between patient and healthcare provider and for increasing patients' knowledge and involvement. ${ }^{11}$ Individualized prediction of successful intended VBAC may contribute to personalisation of the decision-making process and could place potential risks into context. Although there are several prediction models ${ }^{6,12,13}$ and two PtDAs ${ }^{14,15}$ on mode of birth after CS, there has as yet been no published integration of a prediction model with a PtDA. In this study we aim to develop and pilot test a PtDA for mode of delivery after previous CS that integrates personalised prediction of successful VBAC with clarification and elicitation of patient preferences and evidence-based information. 


\section{Methods}

\section{Construction of the PtDA}

The PtDA was developed using the systematic process illustrated in Figure 8.1.16

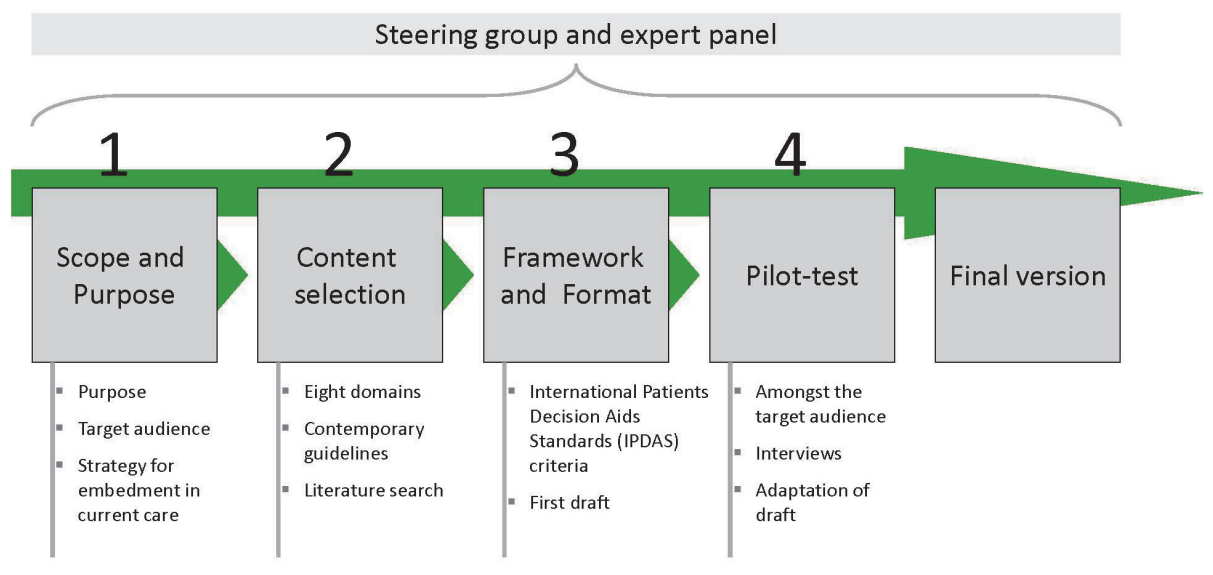

Figure 8.1 The process of developing the patient decision aid (PtDA) consisted of four steps: (1) definition of the scope and purpose of the PtDA, (2) content selection, (3) selection of the framework, format and decision support methods, and (4) pilot testing (i.e. alpha testing) of the prototype amongst the target audience. ${ }^{16}$ The development process was supervised by a steering group and a nationwide expert panel. The steering group consisted of two obstetricians and experts in epidemiology, shareddecision making and guideline implementation. The expert panel consisted of nine independent obstetricians, one midwife and one professor of midwifery science.

\section{Scope and purpose}

Its purpose was to improve decision-making on mode of birth after previous CS, as part of a guideline implementation strategy for the improvement of current Dutch care on CS. ${ }^{17}$ The target audience, i.e. future users of the PtDA, were defined as pregnant women with a history of one previous CS without a contraindication for an intended VBAC. Contraindications were determined in accordance with current international guidelines on pregnancy and childbirth ${ }^{1-5}$ and included: previous uterine rupture or other relevant uterine scar or a contraindication for vaginal labour such as malpresentation or placenta praevia. ${ }^{1-4}$ It was decided not to include information in the PtDA on twin or breech pregnancies or in cases when more than one CS had already been performed, as we considered counselling in these cases to be essentially different. 


\section{Content selection}

Based on current international guidelines on pregnancy and childbirth after CS, ${ }^{1-5}$ literature and expert opinion, we identified eight domains to be important for decision-making on mode of birth after prior CS: (1) risks and (2) benefits of intended VBAC and ERCS, (3) induction of labour, (4) the consequences of the decision for future pregnancies, (5) predictors of successful intended VBAC, (6) the woman's preference for a vaginal or CS delivery, (7) the possibility of a birth plan (i.e. a document containing the woman's preferences when giving birth) and (8) previous birth experiences. Current guideline recommendations dictated the level of detail of the medical contents of domains 1 to 5.1-5 Current data on risk estimates was included, based upon the principle of best available evidence. For domain 5, a prediction model was included that estimates the probability of successful intended VBAC. This prediction model was developed by our research group in a western European cohort of women with high rate of intended VBAC. As prescribed by current methodological guidelines for prediction research, we selected potential predictors based on clinical reasoning and evidence from the literature. ${ }^{18,19}$ The final set of predictors included in the model are: estimated fetal weight $\geq 90$ th percentile, previous CS due to failure to progress, previous vaginal delivery, induction of labour, pre-pregnancy body mass index and ethnicity. The development and internal validation of this prediction model is described elsewhere. ${ }^{20}$ Domains 6, 7 and 8 were elaborated based on expert opinion and a review of the literature.

\section{Selection of framework and format}

Based on the content selection, a practical framework was derived that consisted of the above mentioned eight domains. A prototype of the PtDA was developed in accordance with the international quality criteria of the International Patients Decision Aid Standards (IPDAS). ${ }^{21}$ More information regarding the selection process for the framework and the format can be found in Appendix S1 in the Supporting Information.

\section{Pilot testing and revision}

Pilot testing (i.e. alpha testing) comprised several rounds of review and revision of the prototype based on information gathered during development (MEC number 12-4-034). We recruited women in 2012 at an obstetric outpatient clinic of a Dutch university teaching hospital. Inclusion criteria were: pregnancy with a history of one CS and a current singleton cephalic pregnancy and a minimum age of 18 years. Women with a contraindication for an intended VBAC or who could not read Dutch were excluded. The primary outcome measures were usability, clarity, relevance of the content and relevance of the PtDA in general. After informed consent was obtained, women participated in a 30-minute face-toface audio-recorded interview. The first part of the interview was unstructured according to 
the 'think aloud method'.22 This method allowed us to obtain data using observation and without interrupting participants' thinking processes. The second part of the interview was conducted using a questionnaire. Issues of particular interest to us were the opinions of women on the stage at which the PtDA was introduced, opinions about the prediction model, whether there was a minimum outcome of the prediction model that women considered acceptable when deciding to try for a VBAC, unwanted adverse effects of the PtDA (such as increased anxiety), and opinions regarding the way in which risk was communicated and the preference elicitation tool.

The interviews were transcribed verbatim and translated into a thematic framework. Interviews were analysed by two independent researchers (ES and BA or MdK). Data collection and revision of the PtDA was continued over several rounds until data saturation was achieved.

\section{Results}

\section{Construction of the PtDA}

The practical framework, i.e. the outline of the PtDA, was constructed on the basis of the selected medical contents and our eight domains. Due to the availability of an evidencebased report on birth after CS by Guise et al., ${ }^{6}$ the quantification of risk was mainly based on data from meta-analyses. We included four additional papers because they either provided data that were more applicable for the target audience of the PtDA, based on population characteristics, ${ }^{23}$ were recently published and therefore not included in Guise et al.'s report ${ }^{6}$ or were included in that report but not in the form of a meta-analysis. ${ }^{24}$ The risk of uterine rupture was shown to range from 2.1 to 15 per 1000; we used 8 per 1000 (for spontaneous onset of labour) as a mean since this was more appropriate for the Dutch setting. This was based on data from a Dutch prospective cohort study ${ }^{23}$ which reported a higher incidence ( 8 to 15 per 1000) of uterine rupture in women who attempt VBAC than was reported by the meta-analysis ${ }^{6}(4.3$ per 1000) and a recent large cohort study by Fitzpatrick et al. (2.1 per 1000). ${ }^{25}$ Our final selection of the risks of intended VBAC and ERCS is shown in Table 8.1.

The PtDA was subdivided into a general introduction, seven steps (chapters) that guide the decision-making process and a supplement that clarifies induction of labour after previous CS. The introduction to the PtDA describes its aim, explains the choice that has to be made regarding mode of delivery and lists the options.

- Step one of the PtDA consists of a question regarding any existing preference for either VBAC or ERCS before reading the PtDA. This enables the woman to identify preliminary thoughts, beliefs and decisions, but this preference can also be discussed with the provider. 
- Step two includes two questions which can be used to evaluate previous birth experiences, fear of vaginal delivery and other external influences which the woman regards as relevant.

- In step three the woman is shown a fact sheet laying out the risks and benefits of both modes of delivery. Risks are illustrated by using pictographs on a scale of per 1000 women. ${ }^{26}$ This level of detail with regard to the risks led us to give careful and extensive consideration to the method of risk visualisation. We derived several formats of the pictographs and elaborated these during pilot testing. Step three also includes the prediction model for successful intended VBAC. This element of the PtDA is shown in Figure 8.2. Providers enter variables into the prediction formula using a computerised calculator and the outcome is noted on the corresponding (empty) pictograph within the PtDA. In order to provide context to the outcome of the prediction model, a mean probability of $70-75 \%$ of successful VBAC is included. ${ }^{1,23}$ All risks and benefits are explained using plain language, pictographs and summaries, in a balanced, unbiased manner.

Table 8.1 Risks of intended vaginal birth after caesarean and elective repeat caesarean section: estimates that were selected for the contents of the patient decision aid based on literature research.

\begin{tabular}{|c|c|c|}
\hline Risk & Intended VBAC & ERCS \\
\hline Maternal mortality & 0.04 per $1000^{6}$ & 0.13 per $1000^{6}$ \\
\hline \multirow[t]{2}{*}{ Severe maternal morbidity* } & 2 per $1000(\mathrm{VBAC})^{7}$ & 8 per $1000^{7}$ \\
\hline & 38 per $1000(\mathrm{CS})^{7}$ & \\
\hline Uterine rupture, general & 8 per 1000 (range 2.0 to 15 per 1000$)^{6,23,24}$ & 0.3 per $1000^{6}$ \\
\hline \multicolumn{3}{|l|}{ Uterine rupture, IOL: } \\
\hline Prostaglandins & 20 per $1000^{6}$ & NA \\
\hline Oxytocin & 11 per $1000^{6}$ & NA \\
\hline \multicolumn{3}{|c|}{$\begin{array}{l}\text { Future pregnancies, risk per number of } \\
\text { previous CS: }\end{array}$} \\
\hline \multirow[t]{2}{*}{ Placenta praevia } & NA & 2: 17 per $1000^{6}$ \\
\hline & & 3: 30 per $1000^{6}$ \\
\hline \multirow[t]{2}{*}{ Placenta accreta } & NA & 2: 6 per $1000^{25}$ \\
\hline & & 3: 21 per $1000^{25}$ \\
\hline \multirow[t]{2}{*}{ Hysterectomy } & NA & 2: 9 per $1000^{25}$ \\
\hline & & $3: 24$ per $1000^{25}$ \\
\hline \multirow[t]{2}{*}{ ICU admission } & NA & 2: 5 per $1000^{25}$ \\
\hline & & 3: 16 per $1000^{25}$ \\
\hline \multirow[t]{2}{*}{ Blood transfusion $\geq 4$ units } & NA & 2: 8 per $1000^{25}$ \\
\hline & & 3: 16 per $1000^{25}$ \\
\hline \multirow[t]{2}{*}{ Operative injury** } & NA & 2: 13 per $1000^{25}$ \\
\hline & & $3: 24$ per $1000^{25}$ \\
\hline Neonatal mortality & 1.3 per $1000^{6}$ & 0.5 per $1000^{6}$ \\
\hline Severe neonatal morbidity & \multicolumn{2}{|c|}{ No evidence for a significant difference in the long term ${ }^{6}$} \\
\hline
\end{tabular}

*Composite of uterine rupture, hysterectomy and surgical injury; **Composite of cysthotomy, bowel injury, uretheral injury and bowel obstruction. CS, caesarean section; VBAC, vaginal birth after caesarean section; ERCS, elective repeat CS; IOL, induction of labour; ICU, intensive care unit; NA, not applicable. 
What is my chance of achieving a vaginal birth?

The total risk of complications is smaller for women who have a vaginal delivery. About 70 to 75

percent of the women who start labour with the intention to have a vaginal delivery, indeed

achieve a vaginal delivery.

Calculator: your individual chance of a vaginal delivery ( $>32$ weeks gestational age)

For some women, their individual chance of a vaginal delivery is higher or lower than the general

mean of 70 to 75 percent. After 32 weeks of gestational age we can calculate your individual

chance of a successful vaginal birth based on your medical history and current medical condition.

Your gynaecologist will calculate your individual chance.

If you choose for an intended vaginal delivery, your chance of a successful vaginal

delivery is:

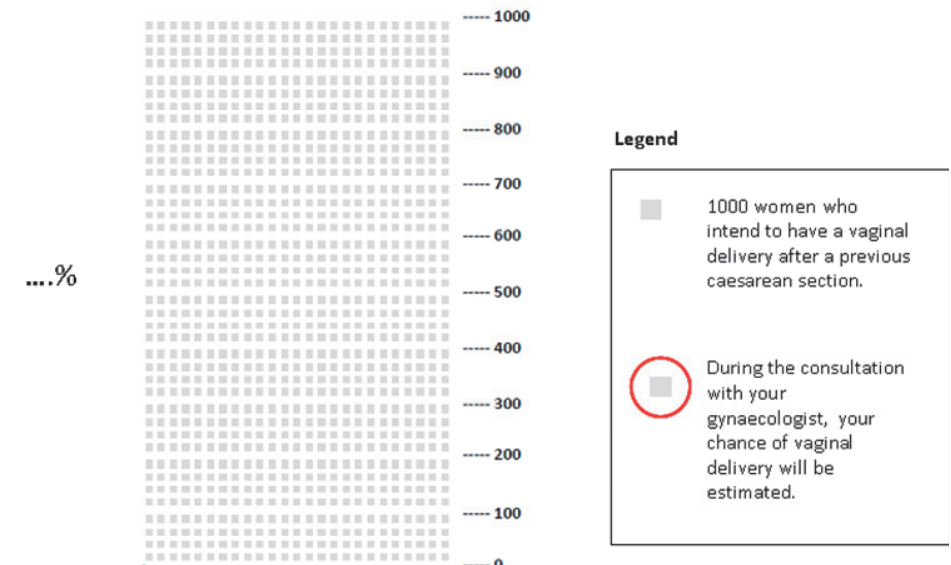

When labour starts before the due date, the chance of a successful vaginal delivery might be increased. However, when labour needs to be induced, the chance of a successful vaginal delivery is, to some extent, decreased. Therefore, when the medical conditions change, the calculator needs to be re-entered.

Figure 8.2 Prediction model for successful intended vaginal birth after caesarean. This is the element of the patient decision aid that explains the prediction model and can be used to show the individualized probability of successful intended vaginal birth after caesarean.

Step four involves a preference elicitation tool, which was designed as a list of statements that women (and their partners) can consider to clarify their values and thoughts around several aspects of the decision. The statements can be scored on a four-point Likert scale to show level of agreement with the statements. Statements were derived from the entire contents of the PtDA and were completed with data from qualitative studies of women's 
views on deciding about mode of birth after CS.27,28 The preference elicitation tool can be viewed in Appendix S2.

- Step five of the PtDA explains the option of making agreements on the delivery (birth plan), such as an epidural analgesia. This step facilitates discussion of a birth plan between the woman and healthcare provider.

- Step six requires is preliminary decision.

- Step seven is aimed to encourage the woman and healthcare provider to agree on follow-up.

- In the supplement, induction of labour after CS is considered: risks and methods of induction are explained and this encourages recalculation of the probability of successful intended VBAC.

\section{Pilot testing and revision}

\section{Pilot one}

In total, 16 eligible women with a history of one previous CS were included in the first pilot. The baseline characteristics are described in Table 8.2. The mean age of the participants was 33 years; all were native Dutch speakers and 14 out of $16(87 \%)$ had the median socioeconomic status (SES). Pilot one consisted of three rounds in which interviews were conducted and the PtDA was revised. The majority of the revisions concerned wording and clarity. Many women stated that they experienced a lack of information within the current care set-up. With regard to timing of exposure to the PtDA, a range of 12 weeks gestational age to 36 weeks gestational age was considered appropriate. Further, 15 out of 16 participants thought that the outcome of the prediction model for successful intended VBAC helped their decision-making process. Two women would choose an ERCS if their predicted probability of successful VBAC was $50 \%$ or less, and one woman set the cut-off value at $60-65 \%$. Thirteen women noted that they could not define a minimum outcome of the prediction model that they considered acceptable for trying a VBAC.

In general, all women thought that the PtDA was clear and informative. The PtDA was considered relevant by all participants. The stepwise approach of the decision-making process was repeatedly cited as a positive point. Also, most women felt that the PtDA was complete, balanced and objective. An aspect that was repeatedly mentioned to require improvement was the pictographs of the health outcomes. In rounds one and two, many women stated that one or more of the pictographs did not clarify the risks. They thought that the pictographs were distracting and added nothing to the decision-making process. In the third round, women thought that the presented figures were clear, but two out of seven women still thought that the pictures could be omitted. 
In the third round of pilot one, no new information was obtained. Subsequently, we presented the PtDA to an expert panel for critical review and comments. Eight out of 11 experts approved this version. However, three out of 11 experts expressed concerns regarding the presentation of risks. A new outline was suggested for the pictographs that involved using cubes instead of pregnant women and means instead of ranges. ${ }^{26}$ Based on this feedback, the PtDA was revised and this new version was tested in pilot two.

\section{Pilot two}

Nine eligible women were included. The baseline characteristics are summarized in Table 8.2. The mean age of the participants was 36 years; eight out of nine $(89 \%)$ women were native Dutch speakers and four out of nine (44\%) had the median SES.

Table 8.2 Baseline characteristics of pregnant women with a history of one caesarean section who participated in pilot testing of the patient decision aid.

\begin{tabular}{lcc}
\hline Characteristic & Pilot one $(n=16)$ & Pilot two $(n=9)$ \\
\hline Maternal age (years, mean \pm SD) & $33 \pm 3$ & $36 \pm 4$ \\
Native Dutch speakers $(n, \%)$ & $16(100)$ & $8(89)$ \\
Socio-economic status* $(n, \%)$ & & $1(11)$ \\
High & $2(13)$ & $4(44)$ \\
Median & $14(87)$ & $2(22)$ \\
Low & $0(0)$ & $2(22)$ \\
Unknown & $0(0)$ & $5(56)$ \\
Previous CS $(n, \%)$ & & $4(44)$ \\
Emergency CS & $10(63)$ & $1(11)$ \\
Planned CS & $6(38)$ & \\
Previous vaginal delivery $(n, \%)$ & $2(13)$ & $5(56)$ \\
Preferred mode of birth & $12(75)$ & $4(44)$ \\
Intended VBAC & $4(25)$ & \\
ERCS & & \\
\hline
\end{tabular}

SD, standard deviation; CS, caesarean section; VBAC, vaginal birth after caesarean section; ERCS, elective repeat CS. *Derived from post-code related socio-economic status scores, based on data on income, educational level and profession.

Pilot two resulted in minor additional changes regarding the wording and clarity of the PtDA. The revised pictographs were considered clear, and seven out of nine women thought that the pictographs supported the information in the tables. Eight out of nine women thought that the PtDA was relevant. After pilot two, no major revisions were required. After final adjustments were made, the PtDA was presented to the expert panel. The PtDA was approved by all members of the expert panel.

\section{IPDAS criteria}

We used the checklist of the IPDAS collaboration ${ }^{21,29}$ to estimate the quality of the developed PtDA. Of the 64 items on the checklist, 50 quality criteria were applicable to our 
study based on the scope of the PtDA. The final version of the PtDA met 39 out of the 50 applicable IPDAS criteria (78\%). ${ }^{21,29}$ With regard to content, all 23 criteria were met. For the domain development process, we met 16 out of 20 criteria. The four criteria that we did not meet were whether the PtDA is written according to a validated readability score (10.4) and if the PtDA is understood by those with limited reading skills (10.6). Though we pilot tested the PtDA, we did not actually field test (i.e. beta test) the PtDA in the current study (1.4, 1.5). The effectiveness of the PtDA was not evaluated in the current development study; therefore we have not yet met the seven criteria (12.1-12.8) concerning effectiveness.

\section{Discussion}

\section{The main findings}

The current study provides insight in the systematic process of developing a decisionsupport tool by means of interdisciplinary cooperation of healthcare professionals and the critical input of future users, i.e. women and healthcare providers. The agreed format is a booklet with a stepwise approach for decision-making. Opinions on the stage at which women should be introduced to the PtDA varied widely between individual women and providers. Therefore, we agreed on introduction at any gestational age before 36 weeks based on the needs of the woman concerned. The PtDA is evidence based and its content is in agreement with current recommendations and guidelines. Hence, this PtDA could be seen as a guideline implementation tool. The quantification of risks was mainly based on data from meta-analyses. Though no studies on this topic with a grade A level of evidence were available, many large cohort studies provide insight into relevant risks. The need for structured information was confirmed, as women indicated that clear and relevant information is currently hard to find. Women emphasized the clarity of the step-wise approach to decision-making and of the preference elicitation tool. As risks had to be shown on a scale per 1000 women, several versions of the pictographs had to be pilot tested. Thanks to the critical input of women and healthcare experts, we established a format that was usable and clear. The pilot study confirmed that women value the use of a prediction model for decision-making, though many women could not define a minimum outcome of the prediction model that they considered acceptable for trying a VBAC. However, our results cannot directly show the impact that application of the prediction model will have on women's choices. Actual application of the prediction model requires the woman and the gynaecologist to interpret the outcome of the prediction model together, and this is necessary in order to measure its effect. Based on the results of pilot testing, it is likely that this PtDA supports the decision-making process, but results on the effectiveness of the strategy have to be further explored. 


\section{Strengths and weaknesses}

We developed the PtDA according to a thorough systematic approach. Quality control was performed using critical input and review of the expert panel. The opinions of 25 women amongst the target audience were taken into account during development. However, readers may question the representativeness of the pilot study since it was performed in just one tertiary hospital and only included a small number of women with a low SES. We acknowledge that results should be confirmed in other settings with a larger study population. Other drawbacks of our design are that we did not specifically test the PtDA embedded within the routine clinical pathway (field testing). Also, we did not target women with limited reading skills, and this may have influenced attitudes about the pictographs, for example. Therefore, the results of this pilot study should be interpreted with caution and should be confirmed and complemented by the results of a larger, prospective trial.

\section{Interpretation}

Two other PtDAs on mode of birth after CS have been published. The PtDA of Shorten et al. ${ }^{14}$ was published in 2004 and comprised a booklet with a value clarification exercise. A systematic review conducted by Vlemmix et al. estimated the agreement of published obstetrics PtDAs with the IPDAS criteria. ${ }^{30}$ According to this review, the PtDA of Shorten et al. meets 34 of 50 IPDAS criteria. ${ }^{30}$ Another PtDA, developed by Emmett et al. and published in $2007,{ }^{15}$ comprised a computerised information programme with a decision analysis tool. This PtDA meets 29 of 50 IPDAS criteria. ${ }^{30}$ In the current study, we were able to meet 39 out of 50 applicable IPDAS criteria, which compares well with other PtDAs in obstetrics, ${ }^{31}$ especially when taking into account that effectiveness criteria $(n=7)$ will be further explored in ongoing research. Our PtDA has similarities to other available PtDAs on delivery after $\mathrm{CS}^{14,15}$ in the choice of key issues that are addressed. However, our PtDA also provides a new decision-making element, namely the integration of an individualised estimation of intended VBAC success with evidence-based information. We consider the prediction model to be applicable to other western European populations with comparable VBAC rates and ethnicities. With regard to other specific information on risk estimates, the PtDA should be evaluated along local guidelines and literature since differences in obstetric policy and case-mix may impair the validity of the data. Hence, the PtDA can be adapted for different populations.

We expect that the introduction of our PtDA in current care will enhance the involvement of women in decision-making on mode of delivery after CS. Subsequently it is hoped that this will reduce decisional conflicts and increase patient satisfaction. Application of the prediction model might alter birth preferences and reduce emergency CS; if this effect is observed we would expect that a reduction in major maternal and neonatal morbidity. 
Before this PtDA can be incorporated into current care, its effectiveness needs to be ascertained in a controlled prospective trial. Currently, a prospective trial amongst 12 Dutch hospitals is being carried out in order to determine its effectiveness in terms of CS rates, adherence to guidelines, decisional conflict, patient satisfaction, feasibility and costs (SIMPLE TRIAL: NCT00800384).

\section{Conclusion}

This paper provides insight into the systematic development of a PtDA that aims to enhance the involvement of women in decision-making on mode of delivery after CS. It has uncovered the main issues around the involvement of women in decision-making, namely the medical and nonmedical items that women and experts consider relevant for decisionmaking, the method of communicating risks, the additive value of using a prediction model for successful intended VBAC and the stage at which women are introduced to the PtDA. The need for a healthcare strategy for mode of delivery after CS was confirmed. In the current format the PtDA is applicable for western European countries, and specific information with regard to risk estimates may be adapted for different populations. The pilot study suggests that the PtDA and the included prediction model support the decisionmaking process. Its effectiveness in terms of CS rates, adherence to guidelines, decisional conflict, patient satisfaction, feasibility and costs will be further examined in a prospective controlled trial.

\section{Appendix S1. Selection of the framework and format of the 'PtDA'}

Based on the content selection, a practical framework was derived that consisted of the defined eight domains. Several examples of existing PtDAs were studied in order to select the appropriate format and decision support method. An important criterion for selection of format and decision support method was usability and applicability within current care. After construction of the framework and selection of the format and decision support methods, a prototype of the PtDA was developed in accordance with the international quality criteria of the International Patients Decision Aid Standards (IPDAS). ${ }^{21}$ The practical framework, i.e. outline of the PtDA, was constructed based on the selected medical contents. Subsequently, several possible formats were discussed: an online program, a booklet and an option grid. ${ }^{32}$ It was decided that a booklet was most compatible with current care. We used the checklist of the IPDAS collaboration ${ }^{21,29}$ in order to complete the outline of the PtDA and to derive the format of the PtDA. 


\section{Appendix S2. Preference elicitation tool}

This preference elicitation tool of the patient decision aid can be used for value clarification and establishing preferences on both options of mode of birth after prior caesarean section (intended vaginal birth after caesarean or elective repeat caesarean section).

\begin{tabular}{|c|c|c|c|c|}
\hline What is important to you (and your partner)? & 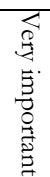 & 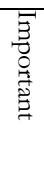 & 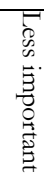 & 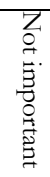 \\
\hline In a vaginal delivery & & & & \\
\hline $\begin{array}{l}\text { I feel more involved in the birth of my baby } \\
\text { I will experience a normal childbirth } \\
\text { The birth will occur the natural way } \\
\text { I will experience pain/contractions during labour } \\
\text { I have less control of the labour process } \\
\text { Episiotomy or tearing might happen } \\
\text { There is a risk of an (emergency) caesarean section } \\
\text { The quantity of my individual chance of a successful vaginal delivery }\end{array}$ & & & & \\
\hline In a caesarean section & & & & \\
\hline $\begin{array}{l}\text { I have more control of when and how the delivery takes place. } \\
\text { There is definitely an operation } \\
\text { I will experience pain after the surgery } \\
\text { There is a higher chance of complications like thrombosis, infection and } \\
\text { bleedings. }\end{array}$ & & & & \\
\hline Severe risks & & & & \\
\hline $\begin{array}{l}\text { In a vaginal delivery, there is a larger risk of a rupture of the scar in the uterus } \\
\text { (uterine rupture). } \\
\text { The overall chance of severe complications for the mother in a vaginal delivery, is } \\
\text { smaller. } \\
\text { In a caesarean section, there is a little higher chance of death of the mother. } \\
\text { For a vaginal delivery and a caesarean section, the chance of permanent injuries } \\
\text { for the baby is the same. } \\
\text { In a caesarean section, the chance of death of the baby is a little smaller. } \\
\text { Every new caesarean section increases risks for a future pregnancy. }\end{array}$ & & & & \\
\hline After a vaginal delivery & & & & \\
\hline $\begin{array}{l}\text { I will probably recover faster. } \\
\text { I will probably return to home earlier. }\end{array}$ & & & & \\
\hline Other important considerations & & & & \\
\hline
\end{tabular}




\section{References}

1. Dutch Society of Obstetrics and Gynaecology. Guideline: Pregnancy and Childbirth after previous caesarean section. 2010.

2. RCOG. Birth after previous caesarean birth. Green-top Guideline. 2007;No. 45.

3. SOGC. Guidelines for Vaginal Birth After Previous Caesarean Birth. J Obstet Gynaecol Can. 2005(155):89: 319-31.

4. ACOG. Vaginal birth after previous cesarean delivery. ACOG practice bulletin. 2010(no. 115).

5. National Institutes of Health Consensus Development Conference Statement vaginal birth after cesarean: new insights March 8-10, 2010. Semin Perinatol 2010; 34(5): 351-65.

6. Guise JM, Eden K, Emeis C, et al. Vaginal birth after cesarean: new insights. Evid Rep Technol Assess (Full Rep) 2010; (191): 1-397.

7. McMahon MJ, Luther ER, Bowes WA, Jr., Olshan AF. Comparison of a trial of labor with an elective second cesarean section. N Engl J Med 1996; 335(10): 689-95.

8. Shorten A, Shorten B. The importance of mode of birth after previous cesarean: success, satisfaction, and postnatal health. J Midwifery Womens Health 2012; 57(2): 126-32.

9. Shorten A, Shorten B, Keogh J, West S, Morris J. Making choices for childbirth: a randomized controlled trial of a decision-aid for informed birth after cesarean. Birth 2005; 32(4): 252-61.

10. Fawsitt CG, Bourke J, Greene RA, Everard CM, Murphy A, Lutomski JE. At What Price? A CostEffectiveness Analysis Comparing Trial of Labour after Previous Caesarean versus Elective Repeat Caesarean Delivery. PLoS One 2013; 8(3): e58577.

11. Stacey D, Bennett CL, Barry MJ, et al. Decision aids for people facing health treatment or screening decisions. Cochrane database of systematic reviews 2011; (10): CD001431.

12. Grobman WA. Rates and prediction of successful vaginal birth after cesarean. Semin Perinatol 2010; 34(4): 244-8.

13. Eden KB, McDonagh M, Denman MA, et al. New insights on vaginal birth after cesarean: can it be predicted? Obstet Gynecol 2010; 116(4): 967-81.

14. Shorten A, Chamberlain M, Shorten B, Kariminia A. Making choices for childbirth: development and testing of a decision-aid for women who have experienced previous caesarean. Patient Educ Couns 2004; 52(3): 307-13.

15. Emmett CL, Murphy DJ, Patel RR, et al. Decision-making about mode of delivery after previous caesarean section: development and piloting of two computer-based decision aids. Health Expect 2007; 10(2): 161-72.

16. Coulter A, Stilwell D, Kryworuchko J, Dolan Mullen P, Ng C, van der Weijden TA. systematic development process for patient decision aids. . Prov accepted BMC Med Infor Dec Mak 2013; ;Suppl.

17. Melman S, Schoorel EN, Dirksen C, et al. SIMPLE: implementation of recommendations from international evidence-based guidelines on caesarean sections in the Netherlands. Protocol for a controlled before and after study. Implement Sci 2013; 8: 3.

18. Steyerberg E. Clinical Prediction Models. New York: NY: Springer; ; 2009.

19. Harrell F. Regression Modeling Strategies. New York: NY: Springer; 2001.

20. Schoorel ENC, van Kuijk SMJ, Melman S, Nijhuis JG, Smits LJM, Aardenburg R. Personalized estimation of successful intended vaginal delivery after caesarean: the development of a Western-European population based prediction model Submitted for publication, BJOG, themed issue 2013.

21. Elwyn G, O'Connor A, Stacey D, et al. Developing a quality criteria framework for patient decision aids: online international Delphi consensus process. Bmj 2006; 333(7565): 417.

22. Lundgren-Laine H, Salantera S. Think-aloud technique and protocol analysis in clinical decision-making research. Qualitative health research 2010; 20(4): 565-75.

23. Kwee A, Bots ML, Visser GH, Bruinse HW. Obstetric management and outcome of pregnancy in women with a history of caesarean section in the Netherlands. Eur J Obstet Gynecol Reprod Biol 2007; 132(2): 171-6.

24. Silver RM, Landon MB, Rouse DJ, et al. Maternal morbidity associated with multiple repeat cesarean deliveries. Obstet Gynecol 2006; 107(6): 1226-32.

25. Fitzpatrick KE, Kurinczuk JJ, Alfirevic Z, Spark P, Brocklehurst P, Knight M. Uterine rupture by intended mode of delivery in the UK: a national case-control study. PLoS Med 2012; 9(3): e1001184. 
26. Zikmund-Fisher BJ, Ubel PA, Smith DM, et al. Communicating side effect risks in a tamoxifen prophylaxis decision aid: the debiasing influence of pictographs. Patient Educ Couns 2008; 73(2): 209-14.

27. Kaimal AJ, Kuppermann M. Understanding risk, patient and provider preferences, and obstetrical decision making: approach to delivery after cesarean. Semin Perinatol 2010; 34(5): 331-6.

28. Emmett CL, Montgomery AA, Murphy DJ. Preferences for mode of delivery after previous caesarean section: what do women want, what do they get and how do they value outcomes? Health Expect 2011; 14(4): 397-404.

29. Elwyn G, O'Connor AM, Bennett C, et al. Assessing the quality of decision support technologies using the International Patient Decision Aid Standards instrument (IPDASi). PLoS One 2009; 4(3): e4705.

30. Vlemmix F, Warendorf JK, Rosman AN, et al. Decision aids to improve informed decision-making in pregnancy care: a systematic review. BJOG 2013; 120(3): 257-66.

31. Knol F, Boelhouwer J, Veldheer V. Neighbourhood status development in the Netherlands 1998-2010. The Netherlands institure for social research 2012.

32. Elwyn G, Lloyd A, Joseph-Williams N, et al. Option Grids: shared decision making made easier. Patient Educ Couns 2013; 90(2): 207-12. 


\section{Chapter 9}

Summary and General discussion 


\section{Summary and General discussion}

The first part of this thesis comprises the evaluation of current care on caesarean sections (CS) in The Netherlands (chapters 2, 3 and 4). In concordance with the analysis of current care, the second part of the thesis subsequently presents the development of a tailored guideline implementation strategy aiming to improve current care (chapters 5, 6, 7 and 8). In this chapter, the background and main findings of the reported studies are summarized. Subsequently, the interpretation of the results and the chosen methodology will be discussed. Also, this chapter describes the results of this project in terms of implications for healthcare. The chapter finalizes with recommendations for further research and the general conclusions.

\section{Summary}

\section{Background}

In part 1 of this thesis, current practice of CS in The Netherlands is discussed. Optimal care regarding both preventive measures and decision making with regard to CS is provided in contemporary international obstetrical guidelines.1-3 However, to what extent these guidelines are incorporated in current care is not evident. Several studies showed that worldwide CS rates are dramatically increasing., ${ }^{4} 5$ Disconcerting is that the main part of this rise can be explained by an increase in CS rate amongst the relatively 'low risk pregnancies' ${ }^{6}$ A CS leads to a higher risk in following pregnancies, a risk of operative injury and to higher costs. ${ }^{7-9}$ Hence, an increase in the CS rate is undesirable. The Netherlands used to have a CS rate very close to the rate advised by the World Health Organisation (WHO) of 10-15\%. ${ }^{10,11}$ However, like the international trend, in The Netherlands the CS rate has risen significantly. The rate has increased from $7.4 \%$ to $16.4 \%$ between 1990 and $2013.11,12$ Striking is that the most impressive rise in CS rate is found in 'low risk pregnancies' which are healthy women with a singleton in vertex position at term. ${ }^{13}$ We hypothesized that this development can be attributed to poor guideline adherence. Therefore we aimed to analyse current care using guideline-based quality indicators in order to detect target points for intervention.

In part two of this thesis, pregnancy following CS in The Netherlands is discussed. After a prior CS, women should be counselled on mode of birth in their current pregnancy. The options are an elective repeat caesarean section (ERCS), or a intended vaginal birth after caesarean (VBAC) (i.e. trial of labour (TOL)). International intended VBAC rates have significantly decreased in the past two decades. ${ }^{14}$ Supposed causes for the decreasing intended VBAC rates centre around the safety issue regarding the possible occurrence of 
uterine rupture during labour. ${ }^{14}$ Against potential complications of an intended VBAC are many benefits such as faster recovery, decreased risk of complications in future pregnancies and a lower risk of overall morbidity when an intended VBAC is successful. ${ }^{15}$ Contemporary guidelines state that when no contra-indication for an intended VBAC exists, both options should be offered and discussed. ${ }^{16-18}$ These recommendations are emphasized by the WHO who clearly state that sufficient information should be provided. ${ }^{19}$ As shown in part 1 of this thesis, in current Dutch care, counselling on birth after CS does not occur according to directive guidelines. Also, VBAC rates are decreasing and may decrease further when no intervention-strategy is provided. Focus group interviews amongst Dutch providers showed however that one of the reasons of poor guideline adherence may be that providers fear that involving women in decision-making might lead to an increase of repeat CSs. ${ }^{20}$ Patient interviews on the other hand, showed that women did not feel involved in decision-making. ${ }^{20}$ Hence, based on the results of part 1 of this thesis, we developed a tailored implementation strategy targeting both providers and patients in order to improve current care on birth after CS in The Netherlands. With regard to the contents of the implementation strategy, we chose for a shared decision making tool that incorporates a prediction model for successful intended VBAC. Literature shows that the probability that an intended VBAC will be successful is a key item within decisionmaking as the incidence of maternal morbidity is lowest in women who have a VBAC $(0.2 \%)$ in comparison to emergency CS $(3.8 \%)$ and ERCS $(0.8 \%) .{ }^{21}$ Other items such as the opinions of the providers, women's own prior experiences and expectations, experienced safety and whether information was tailored to their personal situation are also of main importance in decision making. ${ }^{22}$ Consequently, adequate antenatal counselling is essential in order to direct this process. Because of the complex nature of this decision-making process, mode of birth after CS should be discussed within a shared decision making setting. Hence, we hypothesized that implementing the guideline recommendations on birth after CS through a shared decision making (SDM) tool not only leads to higher guidelineadherence but also to 'better quality' decisions. In this context it is also relevant to personalize decision-making by estimation of individual risks of morbidity per mode of birth. We hypothesized that incorporation of a prediction model for successful intended VBAC in decision-making is feasible and would contribute to the decision-making process. We therefore aimed to develop and pilot-test a SDM-tool that incorporates a predictionmodel for pregnant women who face the decision on mode of birth after CS.

\section{Main findings}

We used the framework of Grol et al. ${ }^{23}$ in order to systematically analyse and improve current care on CSs (chapter 2). The framework contained four main steps: 1: to develop guideline-based quality indicators on the decision when to perform a CS; 2: to analyse 
adherence to the quality-indicators in current Dutch obstetrical care; 3: to explore barriers and facilitators for quality-indicator adherence and 4: to develop, execute, and evaluate a strategy in terms of effectiveness, feasibility, and costs. This thesis reports on the results of step 1, 2 and the development and pilot-testing of the strategy to improve current care (step 4 and part 2 of this thesis).

Chapter 3 describes the actual development and measurement of the guideline based quality indicators. We used a systematic RAND-modified Delphi method ${ }^{24,25}$ in order to develop a set of key recommendations that were appropriate for transcription into quality indicators. A final set of 27 CS guideline-based quality indicators was approved by the national expert panel. Analysis of adherence to these indicators within a multi-centre setting uncovered four main categories as possible target for an intervention strategy: 1) suspected fetal distress (frequency $17 \%$ of the population, CS is performed after fetal blood sampling or ST-analysis in 46\%), 2) non-progressive labour (frequency $12 \%$, CS is performed too early in over $75 \%$ ), 3) continuous support during labour (frequency $88 \%$, CS is performed with continuous support in only $37 \%$ of the cases) and 4) previous CS (frequency $12 \%$, with adequate counselling observed at a mere 15\%). We hypothesize that a guideline implementation strategy on these subjects will result in increased guideline adherence, 'better care' and even in a possible reduction of CS without negative impact on the outcome for mother and child.

In chapter 4 we further analysed the quality indicator 'prior CS'. In this study, we compared inter-hospital ERCS rates and VBAC- rates. The intended VBAC rates and actual VBAC rates were corrected for case-mix based on variables that are predictive for VBAC using a backwards stepwise multiple logistic regression analysis. Seventeen representative Dutch hospitals participated. Women with a history of one prior CS without a contraindication for intended VBAC and who delivered in 2010, were included. In total, 763 records were included for analysis, amongst these cases there were 248 (33\%) ERCSs. Five-hundred -fifteen women (67\%) had an intended VBAC of whom $371(72 \%)$ delivered vaginally. After correction for case-mix, the mean observed ERCS rate was $29.8 \% \pm 11.8 \%$ (range $6 \%$ to $54 \%$ ). The mean for case-mix corrected observed successful intended VBAC rate was $71.8 \% \pm 11.1 \%$ (range $52.7 \%$ to $90.0 \%$ ). This data shows that there is a large practice variation between the hospitals, however, for both outcome measures, the larger part of the practice variation remained unexplained. Consequently, we may state that factors on hospital- and provider level also play a significant role in the derivation of practice variation. In chapter $\mathbf{5}$ we aimed to externally validate the two prediction models for successful intended VBAC of Grobman et al. ${ }^{26,27}$ for the Dutch population. These models were derived and externally validated in an obstetrical population in the Unites States (US).28,29 The first model, the 'entry-to-care' model (ETC), incorporates the variables maternal age, pre-pregnancy body mass index (BMI), ethnicity, prior vaginal delivery, prior VBAC and 
prior non-progressive labour. The second model, the 'close-to -delivery' model (CTD) replaces pre-pregnancy BMI with third-trimester BMI and complements estimated gestational age at delivery, hypertensive disease of pregnancy, cervical examination and induction of labour. The two models are designed for women who have a vertex singleton pregnancy and a history of one low-transverse CS and who delivered at term (gestational age $\geq 37$ weeks). Therefore we included only women who met these criteria for validation of the models. Individual probabilities of successful intended VBAC were calculated and subsequently outcome parameters in terms of discriminative capacity and predictive performance were derived. Both the ETC and the CTD models had a reasonable discriminative capacity of $68 \%$ (95\% CI 63-72\%) and 72\% (95\% CI 67-76\%), respectively. Analysis of predictive performance showed no lack of fit. Despite the reasonable performance of the models, we thought that the two Grobman models ${ }^{27,30}$ are less applicable for clinical practice since they include factors that are only known intrapartum. Also, some variables like 'ethnicity' were less applicable in the Dutch and accordingly the West-European population.

Hence, in chapter 6 we present a new prediction model which was derived by combining and improving existing models. This model was aimed to implement as a tool for counselling on mode of birth after CS for a West-European population. In order to reduce the chance of overfitting and to increase external validity of the developed model we preselected predictors based on clinical reasoning and evidence from previous reports. The final model for predicting successful intended VBAC consists of six variables including demographic variables (pre-pregnancy BMI, Caucasian ethnicity), obstetrical history (previous vaginal delivery and previous non-progressive labour) and current pregnancy variables (induction of current labour and estimated fetal weight (EFW) $\geq$ P90). We internally validated the model using bootstrapping. The developed prediction model has reasonable discriminative capacity $(70.8 \%(95 \% \mathrm{CI}=68.6-72.9 \%)$, and is accurate, especially for women with a predicted probability of $65 \%$ and higher. However, whether the model performs better than clinical judgement was still unclear.

Therefore, in chapter 7 we present a study that compares clinicians' predictive performance for successful intended VBAC in terms of calibration and discrimination to our prediction model. In order to compare clinical judgement to the performance of the model we constructed vignettes which were regarded as actual clinical situations. We showed that, in terms of both discriminative and predictive performance, clinicians perform poorly. Moreover clinicians tended to underestimate the actual probability of success.

Finally, chapter 8 presents the development and pilot-testing of the patient decision aid (PtDA). The PtDA is part of the guideline implementation strategy for improvement of care on birth after prior CS. The systematic development process consisted of a construction phase (definition of scope and purpose, selection of contents, framework and format) and a 
pilot-testing phase. The process was supervised by a multidisciplinary steering group and an expert panel. The developed PtDA is a booklet with a stepwise approach for decisionmaking. The PtDA includes unbiased balanced information on mode of birth after CS, a preference elicitation exercise and tailored risk information including the in chapter 6 presented prediction model. Based on the results of pilot testing, it is likely that this PtDA supports the decision-making process.

\section{General Discussion}

\section{Overall interpretation}

This project used the framework of Grol et al. ${ }^{23}$ for systematically analysing and improving care around CSs, using evidence-based guideline recommendations. The developed quality indicators are unique in the fact that they incorporate both antepartum care and intrapartum care. With regard to The Netherlands, we have uncovered the main indicators that contribute to poor care around CSs (guideline-adherence) and that contribute to overall increasing CS rates and overall feto-maternal morbidity (frequencies). The careimprovement strategy should focus on the indicator with the highest impact in terms of possible gain with regard to guideline adherence in combination with a high frequency of occurrence of the situation. One of these indicators in The Netherlands is care for women with a prior CS. In concordance with the increasing CS rates, the group of women pregnant after a CS rises. The indicator on this subject involves counselling on mode of delivery in current pregnancy. We showed that indicator adherence on this subject is alarmingly low. Also, our analysis showed that though in The Netherlands the majority of women have a VBAC, the rates are decreasing. In respect to prior data, the percentage of intended VBAC decreased from $78.2 \%$ to $67.0 \%$ within eight years. ${ }^{31}$ This observation emphasizes that repeat CSs attribute to the national rising CS rates. The large observed practice variation independent of case-mix shows that individual provider preferences and hospital policies instead of contemporary guidelines play a large role within determining mode of delivery after a prior CS. These findings highlight the need for an intervention strategy.

\section{Enhancing patient involvement in decision making}

The main goals of the intervention are to increase guideline-adherence and to enhance patient involvement in decision-making on mode of birth after CS. The second part of this thesis provides an overview of the development of the components of this strategy. Step one was to create a reliable and applicable prediction model for successful intended VBAC. This model is aimed to enhance decision making for both the professional and the patient. Both the validated prediction models and the developed model have reasonable predictive 
and discriminative performance. Clearly, the ideal prediction model would polarise the cohort into two groups: women with a very high predicted probability and women with a very low predicted probability of achieving a VBAC. However, in comparison to other models that predict successful intended VBAC, the performance of both the developed and the validated models is average to good. ${ }^{15}, 32$ Also, the models showed the potential to classify women away from the population mean which increases the chances of clinical usefulness. We chose to implement the developed model since it was more applicable in the Dutch population and it matches the scope of counselling. By comparing the performance of the developed model to the performance of clinicians, we further explored its clinical usefulness. We showed that by applying the model, more reliable risk estimations would be provided to women of our target group. Furthermore, we showed that most clinicians tend to underestimate the probability of successful intended VBAC which may result in more ERCS prone counselling. Hence, implementing the prediction model might not only lead to more reliable counselling but also to less ERCS's.

Step two for enhancing patient involvement in decision making regarding mode of delivery after CS was to develop and pilot-test the SDM-tool. We systematically developed a PtDA that incorporated the prediction model for successful intended VBAC. Two other PtDA's on birth after CS were published. ${ }^{33}, 34$ These PtDA's have similarities to our PtDA with regard to key issues that are addressed. However, we are the first to integrate a prediction model to a PtDA. The pilot-test confirmed that women value the use of a prediction model for decision-making. Based on the result of the pilot-test, we expect that the introduction of our PtDA in current care will enhance the involvement of women in decision-making. Since the PtDA was developed according to current guidelines, it is likely that implementing the PtDA would maximize guideline-adherence. However, the effectiveness of the strategy in terms of feasibility, costs and improvement of guideline-adherence needs to be further explored in a prospective controlled trial.

\section{Methodological issues}

A strong point of our study is that we included a large number of deliveries amongst 21 representative hospitals, hence the data-set is considered representative for The Netherlands. In order to increase the external validity of the study, we developed the indicator-set on a systematic way using contemporary peer-reviewed international guidelines. We consider the developed indicator-set therefore to be appropriate for other settings than The Netherlands. Its international acceptability however, needs to be established since we did not involve international experts in the indicator development process. Since the CS rate in The Netherlands is relatively low in comparison to other countries $^{11}$, it is plausible that this reflects a different mind-set which may impair external applicability and acceptance of the indicator-set. Therefore, before the indicators are applied 
in other countries, a local expert panel should evaluate them and if necessary, adapt the set to the local situation according to a systematic guideline adaptation process. ${ }^{3,35}$

Factors that may impair the internal validity of the guideline-adherence study mainly involve the methods of data-collection. By retrospectively collecting data on guideline adherence using medical charts, we may underestimate indicator-adherence mainly due to recordingbias. ${ }^{36,37}$ Also, it has also been shown that in particular with regard to physical examination, false positives have been reported in charts. ${ }^{37,} 38$ Furthermore, results may be biased due to misinterpretation from the charts. These factors may impair the internal validity of our study. However, all methods of measuring quality of care implicate bias to a certain level. The chosen method allowed us to obtain information on both frequencies and adherence, which implied to be an effective method to determine the focus of the implementation strategy. Also, due to the increasing availability of electronic patient files, we think that it is likely that chart review becomes a more reliable method of data-collection on quality of care. Furthermore, because data collection was performed by trained research nurses, the probability of misinterpretation was minimized. The subsequent performed barrier study allowed us to further analyse the observed non-adherence in depth.

The practice variation study supports the results of the guideline-adherence study. In this study we showed that guideline-adherence on counselling is low regardless of correction for case-mix. A strength of this study is that data-selection occurred systematically and involved a representative set of hospitals. The selection process of predictive variables for successful intended VBAC was, in line with contemporary insights within prediction research ${ }^{39-41}$, based on literature and expert opinion. A limitation of the chosen method is that we assume that the developed models are capable of explaining practice variation and that the remaining variation can be attributed to policy. Yet, the remaining variation could also be explained by an incomplete model. Another limitation is that, due to lack of data, we could not further specify the exact amount of practice variation that is derived from factors on hospital- and provider level.

Both of the prediction model studies were carried out within a multicentre setting with a good representation of types of hospitals and geographic regions in The Netherlands which increases external validity of the studies. A factor that may impair internal validity involved the handling of missing data. This element within the methodology was present in all prediction studies since we used one dataset. Fortunately, for most variables there was only a small quantity of missing data. We considered the missing data to be missing at random (MAR) and therefore we were able to limit loss of data by using a multiple imputation strategy. Multiple imputation has shown to be superior to complete case analysis since the 
latter results in a large loss of power and might yield biased parameter estimates. ${ }^{41,} 42$ Also, for the development of the prediction model, we carried out a sensitivity analysis using complete cases only. This analysis showed similar results for both datasets. Hence, we believe that missing data did not lead to radically different results.

Another drawback of both of the prediction studies is that usefulness of both prediction models has not yet been proven in terms of for example cost-benefit and patient satisfaction (i.e. impact analysis) . Performing an impact study is essential for determining actual model performance and clinical usefulness in terms of whether model application changes birth preferences and reduces feto-maternal morbidity. Model application may lead to a different selection of women who choose intended VBAC. This may alter model performance. ${ }^{43}$ Whether this effect actually occurs, should be evaluated in a prospective controlled trial in which the prediction model is applied. However, in line with the results of the pilot study of the PtDA, we think that clinical usefulness of a prediction model for successful intended VBAC is likely. Also the results of the vignette study confirmed this assumption.

With regard to the prediction of successful intended VBAC, we performed the first study that compared clinical judgement i.e. 'skilled intuition' to a prediction model. The main drawback of this type of study-design is whether the hypothetical context of a clinical vignette represents clinical practice. ${ }^{44}$ Hence, though the vignettes were developed through extensive literature research in accordance with a multidisciplinary project group, results should be confirmed in a prospective setting.

After the development of the prediction model, the next step was to incorporate it in counselling on mode of birth. PtDA's have proven to be effective tools for enhancing counselling and for increasing patients' knowledge and involvement. ${ }^{45}$ Our PtDA was systematically developed in accordance with the criteria of the international patient decision aid standards (IPDAS) criteria. ${ }^{46}$ Thanks to the critical input of 25 women of the target audience, an expert panel and a multidisciplinary steering group, we presented a thoroughly reviewed and usable PtDA. A weakness in the design was the chosen setting of the pilot study, by involving only a tertiary hospital and a small sample of women with a low socioeconomic status, generalizability of the results may be too optimistic. The currently ongoing prospective controlled trial in which the PtDA is applied should further explore its usability, clarity and relevance.

\section{Implications for daily practice}

In this project we translated the results of the analysis of current Dutch care on CS into clinical practice by developing a tailored implementation strategy. Accordingly, we 
developed a guideline based PtDA. This PtDA consisted of a booklet including unbiased balanced information on mode of birth after CS, a preference elicitation exercise and tailored risk information including a prediction model for successful VBAC. The target audience of the PtDA is women who are pregnant following CS and their providers. For patients, the PtDA is likely to be an important tool to enhance their involvement in decision making on mode of birth after CS. For providers, usage of the PtDA will facilitate guideline adherence and therefore is likely to result in improvement of quality of care. Hence, the next step is carrying out a multicentre controlled trial in which care including the PtDA is compared to care without the PtDA in terms of guideline-adherence, effectiveness, feasibility and costs. When the PtDA indeed results in 'better care', it could be implemented on a larger scale. Subsequently, the PtDA could be handed out at the hospital, made available on the internet and for example be transformed into an application for smartphones. Also, it could be linked to the current Dutch guideline on birth after CS to increase its availability to providers . ${ }^{16}$ Accordingly, the PtDA could be discussed during consultation with the obstetrician or a specialised nurse. However, this requires a setting that is eligible for use of decision support aids and physicians who are know the concept of SDM. Hesitation of physicians to the use of decision support tools has been described in a systematic review by Gravel et al. ${ }^{47}$ The main barriers for using decision support tools were: time constraints, lack of applicability and the clinical situation. ${ }^{47}$ Also, a recent review by Elwyn et al. ${ }^{48}$, showed that widespread adoption of diverse decision support interventions had not occurred within routine care. The underlying issues preventing widespread adoption of the decision support tools could not be further specified. Hence, implementing the PtDA within routine care will be challenging.

\section{Implications for future research}

The results of our project are promising and raise important issues for future research. First, next to care regarding decision making on mode of birth after CS, we uncovered in total three other main indicators as targets for care improvement on CS in The Netherlands. Hence, an intervention strategy on 'continuous support during labour', 'suspected fetal distress' and 'non-progressive labour' will also beneficial for Dutch care. Second, the effectiveness of the developed tool for guideline implementation on birth after CS should be evaluated. Also its feasibility and costs have to be further assessed in order to maximize the success of the implementation process. Third the validity of the developed prediction model should be further analysed through application. A prospective trial amongst 12 Dutch hospitals has to be performed in order to answer these remaining items, this trial is currently carried out (SIMPLE TRIAL: NCT00800384). A challenge for future research would be to investigate the international applicability of both the prediction model and the PtDA. 


\section{General conclusion}

The results of this thesis lead to the following principal findings:

- The following four subjects were uncovered as a possible target for an intervention strategy in order to improve current Care on both preventive measures as the decision making regarding the performance of a CS in The Netherlands: 'previous CS', 'continuous support during labour', 'suspected fetal distress' and 'non-progressive labour'.

- In The Netherlands, a large practice variation is observed on both the amount of ERCS and successful intended VBAC. This variation can only for the smaller part be explained by case-mix which indicates that local policy plays a more important role.

- External validation of two predictive models developed in the United States revealed an adequate performance within the Dutch population. Yet these models were less applicable for clinical practice since they either include factors that are only known intrapartum or included factors that were less applicable in a West-European population.

- We developed a new prediction model for successful intended VBAC for thirdtrimester counselling in a West-European population, the following predictors are included: estimated fetal weight $\geq$ P90 in the third trimester, previous non-progressive labour, previous vaginal delivery, induction of labour, pre-pregnancy body-mass-index and ethnicity. This model has a good discriminative ability and is well calibrated.

- In terms of both discriminative and predictive performance, clinicians perform poorly in respect to the developed prediction model for successful intended VBAC. Moreover clinicians tended to underestimate the actual probability of success.

- In order to improve care on birth after prior CS, we developed a PtDA. This PtDA includes unbiased balanced information on mode of birth after CS, a preference elicitation exercise and tailored risk information including the prediction model on successful intended VBAC. It is likely that this PtDA supports the decision-making process. After effectiveness of this PtDA is established, it should be implemented in standard care.

\section{References}

1. NICE, 2011, Caesarean section: full guideline. National Insitute for Health and Clinical Excellence: London.

2. National Institute for Health and Clinical Excellence. Clinical Guideline 132: Caesarean Section. London: RCOG, 2011.

3. Fervers B, Burgers JS, Haugh MC, et al. Adaptation of clinical guidelines: literature review and proposition for a framework and procedure. Int J Qual Health Care 2006; 18(3): 167-76. 
4. Declercq E, Young R, Cabral H, Ecker J. Is a rising cesarean delivery rate inevitable? Trends in industrialized countries, 1987 to 2007. Birth 2011; 38(2): 99-104.

5. Gibbons L, Belizan JM, Lauer JA, Betran AP, Merialdi M, Althabe F. Inequities in the use of cesarean section deliveries in the world. Am J Obstet Gynecol 2012; 206(4): 331 e1-19.

6. Brennan DJ, Murphy M, Robson MS, O'Herlihy C. The singleton, cephalic, nulliparous woman after 36 weeks of gestation: contribution to overall cesarean delivery rates. Obstet Gynecol 2011; 117(2 Pt 1): 273-9.

7. Luz Gibbons JMB, Jeremy A Lauer, Ana P Betrán, Mario Merialdi and Fernando Althabe. The Global Numbers and Costs of Additionally Needed and Unnecessary Caesarean Sections Performed per Year: Overuse as a Barrier to Universal Coverage. World Health Report (2010); Background Paper, 30.

8. Souza JP, Gulmezoglu A, Lumbiganon P, et al. Caesarean section without medical indications is associated with an increased risk of adverse short-term maternal outcomes: the 2004-2008 WHO Global Survey on Maternal and Perinatal Health. BMC Med 2010; 8: 71.

9. Villar J, Carroli G, Zavaleta N, et al. Maternal and neonatal individual risks and benefits associated with caesarean delivery: multicentre prospective study. BMJ 2007; 335(7628): 1025.

10. Appropriate technology for birth. Lancet 1985; 2(8452): 436-7.

11. Kramer HM, Kwee A, Bremer HA. [Once a C-section, always a C-section?]. Ned Tijdscbr Geneeskd 2009; 153(4): 136-40.

12. Stichting Perinatale Registratie Nederland. Perinatale Zorg in Nederland 2013. Utrecht: Stichting Perinatale Registratie Nederland.

13. Menacker F, Hamilton BE. Recent trends in cesarean delivery in the United States. NCHS data brief 2010; (35): 1-8.

14. Roberts RG, Deutchman M, King VJ, Fryer GE, Miyoshi TJ. Changing policies on vaginal birth after cesarean: impact on access. Birth 2007; 34(4): 316-22.

15. Guise JM, Eden K, Emeis C, et al. Vaginal birth after cesarean: new insights. Evidence Rep Technology Assess 2010; (191): 1-397.

16. Lee YM, D'Alton ME. Cesarean delivery on maternal request: maternal and neonatal complications. Curr Opin Obstet Gynecol 2008; 20(6): 597-601.

17. Colais P, Fantini MP, Fusco D, et al. Risk adjustment models for interhospital comparison of CS rates using Robson's ten group classification system and other socio-demographic and clinical variables. BMC Pregnancy Childbirth 2012; 12: 54.

18. Farine D, Shepherd D. Classification of caesarean sections in Canada: the Modified Robson criteria. J Obstet Gynaecol Can 2012; 34(10): 976-83.

19. WHO, a declaration on the promotion of patients' rights in Europe. 1994

20. Melman et al. Development and measurement of guidelines-based quality indicators of caesarean section care in the Netherlands: a RAND-modified Delphi procedure and retrospective medical chart review. unpublished data.

21. McMahon MJ, Luther ER, Bowes WA, Jr., Olshan AF. Comparison of a trial of labor with an elective second cesarean section. N Engl J Med 1996; 335(10): 689-95.

22. Kaimal AJ, Kuppermann M. Understanding risk, patient and provider preferences, and obstetrical decision making: approach to delivery after cesarean. Semin Perinatol 2010; 34(5): 331-6.

23. Grol R, Grimshaw J. Evidence-based implementation of evidence-based medicine. Jt Comm J Qual Improv. 1999; 25(10): 503-13.

24. Hermens RP, Ouwens MM, Vonk-Okhuijsen SY, et al. Development of quality indicators for diagnosis and treatment of patients with non-small cell lung cancer: a first step toward implementing a multidisciplinary, evidence-based guideline. Lung Cancer 2006; 54(1): 117-24.

25. Mourad SM, Hermens RP, Nelen WL, Braat DD, Grol RP, Kremer JA. Guideline-based development of quality indicators for subfertility care. Hum Reprod 2007; 22(10): 2665-72.

26. Grobman WA, Lai Y, Landon MB, et al. Can a prediction model for vaginal birth after cesarean also predict the probability of morbidity related to a trial of labor? Am J Obstet Gynecol 2009; 200(1): 56 e1-6.

27. Grobman WA, Lai Y, Landon MB, et al. Development of a nomogram for prediction of vaginal birth after cesarean delivery. Obstet Gynecol 2007; 109(4): 806-12.

28. Costantine MM, Fox K, Byers BD, et al. Validation of the prediction model for success of vaginal birth after cesarean delivery. Obstet Gynecol 2009; 114(5): 1029-33. 
29. Costantine MM, Fox KA, Pacheco LD, et al. Does information available at delivery improve the accuracy of predicting vaginal birth after cesarean? Validation of the published models in an independent patient cohort. Am J Perinatol 2011; 28(4): 293-8.

30. Grobman WA, Lai Y, Landon MB, et al. Does information available at admission for delivery improve prediction of vaginal birth after cesarean? Am J Perinatol 2009; 26(10): 693-701.

31. Kwee A, Bots ML, Visser GH, Bruinse HW. Obstetric management and outcome of pregnancy in women with a history of caesarean section in the Netherlands. Eur J Obstet Gynecol Reprod Biol 2007; 132(2): 171-6.

32. Eden KB, McDonagh M, Denman MA, et al. New insights on vaginal birth after cesarean: can it be predicted? Obstet Gynecol 2010; 116(4): 967-81.

33. Montgomery AA, Emmett CL, Fahey T, et al. Two decision aids for mode of delivery among women with previous caesarean section: randomised controlled trial. BMJ 2007; 334(7607): 1305.

34. Shorten A, Chamberlain M, Shorten B, Kariminia A. Making choices for childbirth: development and testing of a decision-aid for women who have experienced previous caesarean. Patient Educ Couns 2004; 52(3): 307-13.

35. Graham ID, Harrison MB, Brouwers M, Davies BL, Dunn S. Facilitating the use of evidence in practice: evaluating and adapting clinical practice guidelines for local use by health care organizations. J Obstet Gynecol Neonatal Nurs. 2002; 31(5): 599-611.

36. Luck J, Peabody JW, Dresselhaus TR, Lee M, Glassman P. How well does chart abstraction measure quality? A prospective comparison of standardized patients with the medical record. Am J Med 2000; 108(8): 642-9.

37. Shah R, Edgar DF, Evans BJ. A comparison of standardised patients, record abstraction and clinical vignettes for the purpose of measuring clinical practice. Ophthalmic Physiol_Opt. 2010; 30(3): 209-24.

38. Dresselhaus TR, Luck J, Peabody JW. The ethical problem of false positives: a prospective evaluation of physician reporting in the medical record. J Med Ethics 2002; 28(5): 291-4.

39. Harrell FJ. Regression Modeling Strategies. New York NS.

40. Steyerberg EW, Moons KG, van der Windt DA, et al. Prognosis Research Strategy (PROGRESS) 3: prognostic model research. PLoS Med 2013; 10(2): e1001381.

41. Steyerberg EW. Clinical Prediction Models. New York NS.

42. Donders AR, van der Heijden GJ, Stijnen T, Moons KG. Review: a gentle introduction to imputation of missing values. J Clin Epidemiol 2006; 59(10): 1087-91.

43. Van Kuijk SM, Sep SJ, Nelemans PJ, Smits LJ. How long do preconception risk prediction models hold? Influence of selective fertility on model performance. Paediatr Perinatal Epidemiol 2010; 24(6): 602-7.

44. Bachmann LM, Muhleisen A, Bock A, ter Riet G, Held U, Kessels AG. Vignette studies of medical choice and judgement to study caregivers' medical decision behaviour: systematic review. BMC Med Res Methodol 2008; 8: 50 .

45. Stacey D, Bennett CL, Barry MJ, et al. Decision aids for people facing health treatment or screening decisions. Cochrane Dtabase Syst Rev 2011; (10): CD001431.

46. Elwyn G, O'Connor A, Stacey D, et al. Developing a quality criteria framework for patient decision aids: online international Delphi consensus process. BMJ 2006; 333(7565): 417.

47. Legare F, Ratte S, Gravel K, Graham ID. Barriers and facilitators to implementing shared decision-making in clinical practice: update of a systematic review of health professionals' perceptions. Patient Educ Couns 2008; 73(3): 526-35.

48. Elwyn G, Scholl I, Tietbohl C, et al. "Many miles to go ...": a systematic review of the implementation of patient decision support interventions into routine clinical practice. BMC Med Inform Decis Mak 2013; 13 Suppl 2: S14. 
Valorisatie 


\section{Valorisatie}

Dit hoofdstuk beschrijft de valorisatie mogelijkheden van het in dit proefschrift beschreven onderzoek, ofwel hoe de resultaten vertaald kunnen worden naar de klinische praktijk en hoe deze ten gunste komen van het maatschappelijk belang in het algemeen en de zwangere vrouw met een keizersnedelitteken in bet bijzonder.

\section{Relevantie}

Wereldwijd stijgt het aantal keizersneden. Deze toename wordt ook in Nederland gezien. In 2013 werden er in Nederland bijvoorbeeld 170.059 kinderen geboren waarvan er 27.355 kinderen met een keizersnede ter wereld kwamen. ${ }^{1}$ Dit is 16,4 procent van het totaal aantal geboortes. Ter vergelijking, in 1990 was dit percentage nog 7.4\%. ${ }^{1}$ Een logisch gevolg hiervan is dat er tevens een stijging wordt gezien in het aantal vrouwen dat zwanger is na een eerdere keizersnede. Zoals beschreven in hoofdstuk 4 van dit proefschrift heeft anno 2011 ongeveer 11\% van alle zwangeren in Nederland een keizersnede in de voorgeschiedenis, dit betrof in dat jaar ongeveer 19.663 vrouwen. De stijging van het aantal keizersneden is relevant omdat een eerdere keizersnede een grotere kans geeft op morbiditeit en mortaliteit van moeder en kind bij een volgende zwangerschap. Dit komt vooral omdat tijdens de bevalling het litteken open kan 'scheuren' (uterusruptuur). Ook impliceert een toename van het aantal keizersneden dat de kosten van de gezondheidszorg stijgen. Derhalve is een zorgvuldige analyse van de huidige zorg en de mogelijke oorzaken en gevolgen van de stijging van het aantal keizersneden belangrijk. Opvallend is dat er internationaal gezien vooral een stijging wordt gezien bij nullipara (zwangeren die hun eerste kind verwachten) met een 'ongecompliceerde' zwangerschap. ${ }^{2}$ De vraag is dan ook of het onjuist stellen van een indicatie om een keizersnede te doen wellicht bijdraagt aan de stijging van het aantal uitgevoerde keizersneden. Over het stellen van een indicatie om een keizersnede te doen bestaan duidelijke richtlijnen. Zowel de Nederlandse Vereniging voor Obstetrie en Gynaecologie (NVOG), maar ook diverse leidende internationale beroepsorganisaties hebben duidelijke richtlijnen gepubliceerd waarin staat wanneer een keizersnede geïndiceerd is. In hoofdstuk 3 van dit proefschrift analyseerden wij in hoeverre de geldende richtlijnen in Nederland daadwerkelijk werden nageleefd. Het doel hiervan was om de zorg voor zwangeren te verbeteren. De resultaten van hoofdstuk 3 lieten zien dat er in Nederland in de volgende vier 'hoofdgroepen' sprake was van onvoldoende adherentie aan geldende richtlijnen: 1) veronderstelde foetale nood; 2) niet vorderende baring; 3) continuiteit in begeleiding van de bevalling en 4) besluitvorming rondom de manier van bevallen na eerdere keizersnede. De analyse van de huidige zorg resulteerde in de ontwikkeling van een zorgverbeteringsstrategie met betrekking tot besluitvorming rondom de manier van bevallen 
na een eerdere keizersnede. Tijdens een zwangerschap na een eerdere keizersnede dienen zwangere vrouwen gezamenlijk met de zorgverlener een keuze te maken over de manier van bevallen: een intentie tot vaginale bevalling ofwel een primaire keizersnede. Interventie op het gebied van counseling van deze groep vrouwen is nodig omdat uit ons dossieronderzoek bleek dat de zwangeren slechts in 15\% van de gevallen adequaat volgens de richtlijn werden gecounseld. Zoals in hoofdstuk 8 van dit proefschrift beschreven is, bestaat de door ons ontwikkelde interventie uit een geïndividualiseerde keuzehulp die tijdens de zwangerschap gebruikt kan worden om gezamenlijke besluitvorming c.q 'shared decision making' te faciliteren. Of implementatie van deze keuzehulp daadwerkelijk resulteert in een verbetering van de huidige zorg in termen van verbeterde richtlijn adherentie, patiënttevredenheid en kosten, wordt momenteel onderzocht.

\section{Doelgroep}

De in dit proefschrift beschreven studies vallen onder het project SIMPLE (Sectio IMPLEmentatie studie). De doelstelling van dit project is om te analyseren of er juiste indicaties gesteld worden tot het uitvoeren van keizersneden en om dit proces zo mogelijk te verbeteren. De gegevens die zijn gebruikt voor de studies in dit proefschrift zijn verzameld vanuit de huidige reguliere zorg voor zwangere vrouwen in Nederland. De doelgroep van het project is dan ook zeer breed. Alle zwangere Nederlandse vrouwen vallen binnen de doelgroep. Maar de studie is ook van toepassing op zorgverleners zoals gynaecologen en artsen in opleiding tot gynaecoloog omdat zij degenen zijn die de indicatie tot het uitvoeren van een keizersnede stellen. Dit maakt dat deze artsen de mate van richtlijn adherentie deels bepalen. Ook zijn de artsen mede uitvoerders van een zorgverbeteringsstrategie.

In hoofdstuk 8 van dit proefschrift staat de ontwikkeling van een keuzehulp beschreven met daarin een model voor het voorspellen van de kans op een succesvolle vaginale baring na een eerdere keizersnede. Deze twee onderdelen vormen, samen met de training van zorgprofessionals, de basis van de zorgverbeteringsstrategie op het gebied van besluitvorming rondom bevallen na een eerdere keizersnede. De in hoofdstuk 8 van dit proefschrift beschreven zorgverbeteringsstrategie is specifiek gericht op zwangere vrouwen in Nederland met een keizersnede in de voorgeschiedenis en de artsen die deze vrouwen voorlichten. Indien de keuzehulp succesvol geïmplementeerd zou worden en effectief blijkt, kunnen de resultaten mogelijk geëxtrapoleerd worden naar een klinische setting buiten Nederland. Of het ontwikkelde predictiemodel en de keuzehulp hier ook daadwerkelijk geschikt voor zijn, zou aanvullend onderzocht moeten worden. Zoals ook al bleek uit de 
studie die is beschreven in hoofdstuk 5 van dit proefschrift, is een predictiemodel namelijk niet zomaar zonder meer van toepassing en valide in een nieuwe setting. De belangrijkste reden hiervan is dat door verschillende populatie-kenmerken de validiteit van een model kan worden beïnvloed. Ook de 'aanvaardbaarheid' van de inhoud van de keuzehulp onder artsen en patiënten kan per land verschillend zijn. Of verdere uitbreiding van de doelgroep op internationaal niveau mogelijk is, dient dus aanvullend onderzocht te worden.

\section{Innovatie}

De SIMPLE studie is het eerste project dat een indicatoren set ontwikkelde om obstetrische zorg voor indicatiestelling van een keizersnede te meten voor, tijdens en na de partus. Door toepassing van deze set indicatoren werd op unieke wijze de huidige Nederlandse obstetrische zorg rondom keizersneden in kaart gebracht. De vervolgens ontwikkelde keuzehulp met predictiemodel is in het huidige stadium specifiek bedoeld voor Nederland. Innovatief aan deze keuzehulp is dat het de eerste keuzehulp over de manier van bevallen na een eerdere keizersnede is met een predictiemodel. Een voordeel van het toegevoegde predictiemodel is dat de keuzehulp aangepast kan worden aan de individuele patiënt en derhalve uitermate geschikt is om in te zetten voor 'personalized medicine'. En in de hedendaagse geneeskunde wordt deze manier van geneeskunde bedrijven steeds meer de norm. ${ }^{3}$

\section{Implementatie, Planning en Realisatie}

In welke mate de ontwikkelde keuzehulp succesvol geïmplementeerd kan worden in de huidige obstetrische zorg wordt onderzocht in de SIMPLE II studie. De resultaten van dit onderzoek zijn nog niet bekend en zijn dan ook niet beschreven in dit proefschrift. Het doel van de SIMPLE II studie is om te evalueren of het inzetten van de in hoofdstuk 8 beschreven keuzehulp 'bevallen na een eerdere keizersnede' leidt tot een hogere richtlijn adherentie zonder stijging van het aantal (spoed)keizersneden. De interventie werd ingevoerd in zes ziekenhuizen. In zes andere ziekenhuizen werd de reguliere obstetrische zorg gehandhaafd, deze ziekenhuizen dienden als controle. De zorg in combinatie met de keuzehulp zal worden geëvalueerd op het gebied van haalbaarheid en ervaringen. Daarbij zal worden gemeten of het percentage 'vaginal birth after caesarean section' (VBAC) in de studieperiode gelijk blijft en worden de kosten geanalyseerd. Indien deze studie zou uitwijzen dat de zorgverbeteringsstrategie haalbaar en effectief is, kan implementatie op grote schaal in Nederland overwogen worden. Hierbij kan men bijvoorbeeld denken aan het 
koppelen van de keuzehulp aan de NVOG richtlijn 'Zwangerschap en bevalling na een voorgaande keizersnede'.

\section{Referenties}

1. Stichting Perinatale Registratie Nederland. Perinatale Zorg in Nederland 2013. Utrecht: Stichting Perinatale Registratie Nederland.

2. Kwee A, Elferink-Stinkens PM, Reuwer PJ, Bruinse HW. Trends in obstetric interventions in the Dutch obstetrical care system in the period 1993-2002. Eur J Obstet Gynecol Reprod Biol. 2007; 132(1): 70-5.

3. Hayes DF, Markus HS, Leslie RD, Topol EJ. Personalized medicine: risk prediction, targeted therapies and mobile health technology. BMC Med 2014; $12: 37$. 
Nederlandstalige samenvatting 


\section{Nederlandstalige samenvatting}

\section{Achtergrond en doel van het onderzoek}

De keizersnede was oorspronkelijk bedoeld als noodprocedure tijdens de bevalling. De huidige wereldwijde stijging in het aantal keizersneden dat wordt uitgevoerd impliceert onjuiste toepassing van de keizersnede. Meer keizersneden zou kunnen leiden tot meer complicaties voor moeder en kind en tot hogere kosten voor de gezondheidszorg.

Het stijgende aantal keizersneden resulteert tevens in een groeiende groep vrouwen die zwanger zijn na een voorafgaande keizersnede, deze vrouwen hebben dus een litteken in de baarmoeder. De zorg in de zwangerschap en tijdens de bevalling voor deze groep vrouwen vereist een andere aanpak dan voor zwangere vrouwen zonder litteken in de baarmoeder. De reden is dat er een grotere kans op complicaties bestaat tijdens de zwangerschap en bevalling, zoals het scheuren van het litteken. Vrouwen die zwanger zijn na een eerdere keizersnede moeten dan ook worden gecounseld over de manier van bevallen. Hierbij dienen ze de keuze te krijgen tussen een primaire keizersnede en het nastreven van een vaginale bevalling (VBAC). Als een intentie tot VBAC niet slaagt, resulteert dit alsnog in een (spoed)keizersnede. Er bestaan duidelijke evidence-based richtlijnen over wanneer een keizersnede uit te voeren en hoe om te gaan met een zwangerschap na een eerdere keizersnede. Het is echter niet duidelijk of deze richtlijnen op de juiste manier worden gevolgd. Het doel van dit proefschrift is dan ook om in kaart te brengen in hoeverre richtlijnen rondom indicatiestelling en uitvoering van keizersneden in Nederland worden nageleefd door artsen. Vervolgens is het doel om op basis van de resultaten van deze analyse een strategie te ontwikkelen die gericht is op het verbeteren van de huidige zorg.

Dit proefschrift bestaat uit twee delen. Het eerste gedeelte beslaat het meten van de huidige zorg rondom het uitvoeren van keizersneden in Nederland (hoofdstukken 2, 3 en 4). Op basis van de analyse in deel 1 van het proefschrift is een zorgverbeteringsstrategie ontwikkeld. Het proces van het ontwikkelen en uittesten van deze zorgverbeteringsstrategie staat beschreven in het tweede gedeelte van dit proefschrift (hoofdstukken 5, 6, 7 en 8).

\section{Belangrijkste bevindingen}

Zoals beschreven in hoofdstuk 2 is, op basis van het raamwerk van Grol et al., een strategie ontwikkeld om de huidige Nederlandse zorg op het gebied van keizersneden op een systematische wijze te analyseren en te verbeteren. Deze strategie bestaat uit vier 'hoofd stappen' 1: het ontwikkelen van op richtlijnen gebaseerde kwaliteitsindicatoren omtrent de beslissing wanneer een keizersnede uit te voeren; 2: het analyseren van de adherentie aan de kwaliteitsindicatoren in de huidige Nederlandse obstetrische zorg; 3: het onderzoeken van barrières en facilitatoren voor adherentie aan kwaliteitsindicatoren en 4: het ontwikkelen, 
uitvoeren en evalueren van een zorgverbeteringsstrategie met als eindpunten effectiviteit, haalbaarheid en kosten. Dit proefschrift rapporteert de resultaten van stap 1 en 2 , en de ontwikkeling en het pilot-testen van de zorgverbeteringsstrategie (stap 4 en tevens deel 2 van dit proefschrift).

Hoofdstuk 3 beschrijft de ontwikkeling en meting van de op richtlijnen gebaseerde kwaliteitsindicatoren. We hebben hiervoor een gestandaardiseerde methode gebruikt (de RAND-modified Delphi methode) om een set kern-aanbevelingen vast te stellen die geschikt waren voor transcriptie naar kwaliteitsindicatoren. Een uiteindelijke set van 27 kwaliteitsindicatoren werd goedgekeurd door een nationaal expertpanel. Analyse van de adherentie aan deze indicatoren werd uitgevoerd in een multicenter setting en toonde vier belangrijke categorieën als doel voor een mogelijke interventie-strategie: 1) veronderstelde foetale nood (frequentie van voorkomen 17\%, een keizersnede werd uitgevoerd na toepassing van de richtlijn in $46 \%$ van de gevallen); 2) niet-vorderende baring (frequentie van voorkomen $12 \%$, een keizersnede werd in $75 \%$ van de gevallen te vroeg uitgevoerd); 3 ) continue begeleiding tijdens de bevalling (van toepassing op $88 \%$ van de bevallingen, een keizersnede werd uitgevoerd waarbij continue begeleiding tijdens de bevalling aanwezig was in slechts $37 \%$ van de gevallen) en 4) eerdere keizersnede (frequentie van voorkomen $12 \%$, adequate counseling over de manier van bevallen in 15\%). Onze hypothese is dat een richtlijn implementatie-strategie, gericht op deze onderwerpen, zal leiden tot een hogere richtlijn-adherentie, tot 'betere zorg' en zelfs tot een mogelijke reductie van het aantal keizersneden zonder negatieve impact op de uitkomst voor moeder en kind.

In hoofdstuk 4 analyseerden we de kwaliteitsindicator op het gebied van 'zwangerschap na eerdere keizersnede' verder op het gebied van praktijkvariatie. In deze studie vergeleken we in 17 verschillende ziekenhuizen het aantal primaire herhalingskeizersneden dat werd uitgevoerd bij vrouwen die eerder één keizersnede hadden gehad. Tevens werd het aantal succesvolle pogingen tot VBAC onderzocht. De aantallen werden gecorrigeerd voor verschillen tussen patiëntpopulaties (casemix). We analyseerden hiervoor 763 patiëntendossiers, binnen deze groep bevielen 248 (33\%) met een primaire herhalingskeizersnede en 515 vrouwen $(67 \%)$ streefden een VBAC na. De waarden per ziekenhuis, gecorrigeerd voor de patiëntenpopulatie, toonden een grote spreiding: het aantal primaire herhalingskeizersneden was gemiddeld 29.8\% maar met een spreiding tussen de 6 en 54\%. Het aantal succesvolle intenties tot VBAC lag gemiddeld op $71.8 \%$ met een spreiding tussen de ziekenhuizen van $52.7 \%$ tot $90.0 \%$. Deze data laat zien dat er een grote praktijkvariatie bestaat tussen Nederlandse ziekenhuizen op het gebied van bevallen na een eerdere keizersnede. Deze variatie kon niet voldoende verklaard worden door verschillen in patiëntpopulaties van de deelnemende ziekenhuizen. Hierdoor kunnen we stellen dat factoren op het gebied van zorgverleners en op ziekenhuisniveau een belangrijkere rol spelen in het ontstaan van deze praktijkvariatie. 
In hoofdstuk 5 hadden we als doel om twee predictiemodellen te valideren voor de Nederlandse populatie. Dit betrof de Amerikaanse modellen van Grobman et al. waarmee voorspeld wordt wat de kans bij een intentie tot VBAC is dat deze succesvol is (dus daadwerkelijk resulteert in een vaginale baring en niet in een spoedkeizersnede). Het eerste predictiemodel, het 'entry-to-care' model (ETC), bevat de volgende variabelen: leeftijd van de moeder, body mass index (BMI) vóór de zwangerschap, etniciteit, eerdere vaginale bevalling, eerdere VBAC, eerder niet vorderende baring. Het tweede model, het 'close-todelivery' model (CTD), bouwt hierop voort. In dit model is BMI voor de zwangerschap vervangen door BMI in het derde trimester van de zwangerschap en is toegevoegd: zwangerschapsduur ten tijde van de bevalling, hoge bloeddruk tijdens de zwangerschap, parameters van lichamelijk onderzoek tijdens de bevalling (ontsluiting, het verstrijken van de cervix en indaling van het kind) en of er sprake was van het inleiden van de bevalling. De modellen zijn gemaakt voor zwangeren die maximaal één eerdere keizersnede hebben gehad, in de huidige zwangerschap een eenling verwachten in hoofdligging en die à terme bevallen. Om deze reden hebben wij alleen vrouwen geïncludeerd in onze studie die ook aan deze criteria voldeden. Voor iedere vrouw werd de kans op een succesvolle VBAC uitgerekend met beide modellen. Vervolgens werd berekend hoe goed de predictiemodellen presteren in ons cohort. Hiervoor werd gekeken naar onderscheidend vermogen (onderscheid hoge versus lage kans op slagen) en voorspellend vermogen (precisie). Beide modellen hadden een redelijk onderscheidend vermogen en er was sprake van een goede precisie. We concluderen dat beide modellen adequat presteren in de Nederlandse populatie. De modellen zijn echter niet volledig geschikt voor toepassing in de Nederlandse zorg. Dit komt omdat we in Nederland counselen over de manier van bevallen in het derde trimester van de zwangerschap, maar sommige variabelen pas bekend worden tijdens de bevalling. Daarnaast zijn variabelen zoals 'etniciteit' minder van toepassing in de Nederlandse populatie.

Derhalve presenteren wij in hoofdstuk 6 een nieuw predictiemodel, gemaakt op basis van bestaande modellen. Dit model is bedoeld voor counseling omtrent de manier van bevallen na een eerdere keizersnede in het derde trimester van de zwangerschap en is geschikt voor een West-Europese populatie. Om ervoor te zorgen dat het model extern valide is, dus niet alleen maar valide binnen de dataset, werd er een uitvoerig literatuur onderzoek verricht alvorens de variabelen te kiezen die werden opgenomen in het model. Het uiteindelijke model voor het voorspellen van een succesvolle VBAC bevat zes variabelen waaronder demografische variabelen (BMI voor de zwangerschap, Caucasische etniciteit), obstetrische voorgeschiedenis (eerdere vaginale bevalling en eerdere niet vorderende bevalling) en variabelen die bij de huidige zwangerschap horen (inleiding van de bevalling en geschat foetaal gewicht boven het $90^{\text {ste }}$ percentiel). Het ontwikkelde predictiemodel heeft een redelijk onderscheidend vermogen en is accuraat, met name voor vrouwen met een 
voorspelde slagingskans van $65 \%$ of hoger. Echter, of het model beter is dan de 'klinische blik' van de obstetricus is nog niet evident.

Daarom hebben we vervolgens een studie uitgevoerd waarin het ontwikkelde predictiemodel werd vergeleken met de 'klinische blik' (hoofdstuk 7). Om de klinische blik te kunnen vergelijken met prestatie van het predictiemodel werden er vignetten ontwikkeld. In elk vignet werd een klinische casus gepresenteerd op basis waarvan clinici werden gevraagd om de kans op een succesvolle VBAC in te schatten. Vervolgens werd deze kans ook berekend met behulp van het predictiemodel en werden de kansen vergeleken met elkaar en de uiteindelijke uitkomst. Wij toonden aan dat wat betreft onderscheidend vermogen en precisie de clinici niet goed presteerden in vergelijking met het voorspellingsmodel. Clinici neigden naar onderschatting van de daadwerkelijke slagingskans. Tenslotte beschrijft hoofdstuk $\mathbf{8}$ de ontwikkeling en het pilot-testen van de keuzehulp. De keuzehulp is onderdeel van de zorgverbeteringsstrategie ten aanzien van zorg voor zwangeren met een keizersnede in de voorgeschiedenis. Het systematische ontwikkelingsproces van deze keuzehulp bestond uit een constructie fase (bepalen van de doelgroep en het doel van de keuzehulp, selectie van de inhoud en selectie van de weergave van de keuzehulp) en een pilot-test fase. Het proces werd gesuperviseerd door een multidisciplinaire stuurgroep en een expert panel. De ontwikkelde keuzehulp bestaat uit een boekje met daarin een stapsgewijze aanpak van het keuzeproces. De keuzehulp bestaat uit neutrale en gebalanceerde informatie over bevallen na een eerdere keizersnede, een opdracht voor de patiënt om haar wensen en mening ten aanzien van de manier van bevallen te verhelderen en op maat gemaakte informatie inclusief het in hoofdstuk 6 beschreven predictiemodel. De pilot-studie liet zien dat het waarschijnlijk is dat de ontwikkelde keuzehulp bijdraagt aan een beter keuzeproces.

In het afsluitend hoofdstuk 9 worden de belangrijkste bevindingen van dit proefschrift samengevat en bediscussieerd. We stellen dat de ontwikkelde zorgverbeteringsstrategie veelbelovend is voor het verbeteren van de huidige zorg rondom het keuzeproces van de manier van bevallen na een eerdere keizersnede. 
Dankwoord 


\section{Dankwoord}

'Birth after Caesarean, is it SIMPLE?', de afgelopen vier jaar heb ik met veel plezier onderzoek gedaan en ontzettend veel geleerd. 'Simpel' was het zeker niet en dit proefschrift is dan ook tot stand gekomen dankzij de begeleiding en ondersteuning van velen.

Als eerste een woord van dank aan mijn promotor en co-promotoren: Prof dr. J.G. Nijhuis, Dr. H.C.J. Scheepers en Dr. R.P.M.G. Hermens .

Prof dr. J.G. Nijhuis, ik leerde u kennen nog tijdens de A-KO opleiding als projectlid van de SIMPLE studie. Toen ik vervolgens aangesteld werd als promovenda was het een logische stap dat $\mathrm{u}$ mijn promotor werd. Al snel werkten we op uitnodiging van het Nederlands Tijdschrift Obstetrie en Gynaecologie samen aan een klinisch stuk over VBAC. Voor mij de perfecte start van het proefschrift en een goede manier om verdieping te vinden in het onderwerp. Ik wil u vooral bedanken voor uw zeer snelle en scherpe commentaar, betrouwbaarheid en de zeer adequate bijsturing van mijn manier van wetenschappelijk schrijven. Ik vond het altijd erg fijn om zo snel, ondanks uw drukke agenda, zeer nuttig commentaar te krijgen op mijn manuscripten.

Dr. H.C.J. Scheepers, beste Liesbeth, ik wil je bedanken voor de dagelijkse begeleiding van mijn promotietraject. Allereerst wil ik je bedanken voor de kans die je me hebt gegeven om al als student te starten met een $\mathrm{PhD}$ traject. Jouw positieve houding, tomeloze enthousiasme en betrokkenheid heb ik als zeer prettig ervaren en hebben enorm bijgedragen aan het proefschrift dat er nu ligt. Ik heb ontzettend veel van je geleerd, met name op het gebied van het translationele aspect van onderzoek doen. Ik ben trots één van je eerste promovendi te mogen zijn, ik weet zeker dat er nog veel succesvolle promovendi zullen volgen!

Dr. R.P.M.G. Hermens, beste Rosella, ik heb ontzettend veel van je geleerd. Ik wil je bedanken voor je uitgebreide en constructieve feedback op mijn stukken. Je hebt de gave om precies helder te krijgen wat er nog ontbreekt aan een stuk of wat onduidelijk is. Jouw feedback heeft dan ook menig manuscript naar een hoger niveau getild. Ook al zat je op afstand, vanuit Nijmegen heb je via onze (twee)wekelijkse telefonische besprekingen een grote rol gespeeld in mijn ontwikkeling als wetenschapper. Bedankt daarvoor!

De leden van de beoordelingscommissie: Prof. dr. M.H. Prins, Dr. M.J. Faber, Prof. dr. S.A. Scherjon, Prof. dr. M.E.A. Spaanderman en Prof. dr. R.G. de Vries, wil ik bedanken voor de beoordeling van dit proefschrift.

Ik wil graag de leden van de SIMPLE projectgroep bedanken: dr. Luc Smits, prof. dr. Carmen Dirksen, prof. dr. Trudy van der Weijden, dr. Anneke Kwee en prof. dr. Ben 
Willem Mol. Bedankt voor jullie inzet voor het SIMPLE onderzoek en voor jullie uitgebreide feedback op de diverse manuscripten!

Sander van Kuijk, ik heb ontzettend veel van je geleerd. Je hebt een opvallend talent voor onderwijs geven en jouw begeleiding op het gebied van methodologie en statistiek zijn dan ook zeer belangrijk en in zekere zin bepalend geweest voor de totstandkoming van menig artikel in dit proefschrift. Daarnaast wil ik je bedanken voor de fijne en efficiënte samenwerking en alle tijd die je hebt uitgetrokken om met me te brainstormen en me dingen uit te leggen. Ik hoop dat we in de toekomst nog vaker gaan samenwerken!

Emy Vankan en Sonja Melman, uiteindelijk wordt het een driedubbele promotie op de SIMPLE studie! Sonja, jij was tijdens de A-KO een fijne begeleider, dit ging daarna moeiteloos over in 'collega's'. Bedankt voor de fijne samenwerking! Emy, vanaf het begin dat jij erbij kwam klikte het al erg goed en ik ervaar onze samenwerking dan ook als zeer prettig. Jij maakt het laatste stuk van de SIMPLE II studie af en ik heb dan ook met veel plezier en vertrouwen dit stuk verder kunnen loslaten wetende dat het bij jou in goede handen is. Sonja en Emy, ik kijk ook erg uit naar jullie promoties!

Chahinda, wij hebben elkaar leren kennen als collega's bij de gynaecologie, inmiddels is 'collega's' uitgegroeid tot een fijne vriendschap. Bedankt voor alle uurtjes brainstormen op de vierde verdieping van het MUMC, dit heeft zeker zijn vruchten afgeworpen! Ik heb enorme bewondering voor je doorzettingsvermogen en je wetenschappelijk scherpe blik en ben dan ook heel benieuwd waar je over 10 jaar staat, ik weet zeker dat je een mooie carrière tegemoet gaat!

Jannie, wij zijn samen als student begonnen aan de SIMPLE studie. Naast dat we het op persoonlijk vlak al goed konden vinden bleek dat we ook heel fijn en effectief konden samenwerken. Hierdoor heeft de start van mijn promotieonderzoek, waarbij jij me hebt geholpen met het verzamelen van mijn data, een enorme boost gekregen. Bedankt daarvoor!

Ik wil ook 'mijn studenten' bedanken: Brigitte Augustijn, Marleen Alink, Mark de Koning, Dorien Hünen en Anouk van den Bosch. Ik vond het erg leuk om jullie allemaal te begeleiden in het doen van onderzoek en heb fijn met jullie allemaal samengewerkt! Bedankt voor jullie inzet!

Mandy Bronsgeest, beste Mandy, ik wil je heel erg bedanken voor het maken van de omslag van mijn proefschrift, ik ben er erg blij mee! Ik wil ook graag Gerda en Leo bedanken voor jullie steun en interesse in mijn onderzoek en voor het feit dat jullie mij 
Mandy hebben aanbevolen. Een heerlijke week in februari bij Gerda in Spanje heeft ertoe geleid dat ik de laatste puntjes op de i kon zetten voor de afronding van het manuscript waarna dit naar de beoordelingscommissie kon.

Ik wil graag Tiny Wouters bedanken voor het opmaken van dit proefschrift!

Uiteraard verdienen mijn paranimfen hier een uitgebreide vermelding: Hanke en Susan, als 'kwik, kwek en kwak' danwel 'K3' zijn we gedrieën als huisgenoten, studiegenoten en vriendinnen de A-KO met veel plezier maar ook alle drie succesvol doorgekomen. Ook in onze promoties, al gaan deze over zeer verschillende onderwerpen aan verschillende universiteiten, hebben we elkaar gemotiveerd en gesteund: van advies op wetenschappelijk niveau tot aan 'werkmuziekjes', de meer organisatorische aangelegenheden van het promoveren en natuurlijk de nodige ontspanning. Ik ben er trots op dat jullie de $23^{\text {ste }}$ juni naast mij staan als mijn paranimfen. Enorm bedankt voor jullie vriendschap!

Lieve vrienden en vriendinnen. Jullie vriendschap heeft me veel plezier en nieuwe energie gebracht tussen het harde werken door. Bedankt hiervoor en voor de interesse in mijn werk!

Hamid, Susan, Michael en Tanja, ik had me geen fijnere schoonfamilie kunnen wensen. Bedankt voor jullie enthousiasme en brede ondersteuning.

Lief zusje, lieve Esther, ook al zijn we wat betreft onze carrières twee totaal verschillende kanten opgegaan, aan belangstelling en betrokkenheid in mijn werk heeft het je nooit ontbroken. Zo heb je nog vanuit Egypte mijn artikel dat naar de Lancet ging nagekeken op taal, en ben je altijd goed op de hoogte van waar ik mee bezig ben. En uiteindelijk hebben we allebei veel plezier in schrijven en in het doen van wetenschappelijk onderzoek, of dat nou gaat over kwaliteit van zorg of over de revolutie in Egypte... Ik ben ontzettend trots op je en ben benieuwd, je hebt het er al vaak over gehad... wellicht over een paar jaar jouw promotie? En dan wordt natuurlijk de volgende vraag, waar gaat deze plaatsvinden: Zweden, Dubai, Egypte of toch heel misschien Nederland? Ik wens jou en Samy heel veel geluk toe.

Lieve pap en mam, jullie belangstelling en steun zijn voor mij heel belangrijk geweest. Van het begin van het onderzoek tot het eind 'zullen wij het voor je afmaken?', zijn jullie altijd enorm geïnteresseerd geweest en betrokken gebleven. Meerdere gezamenlijke heerlijke weken in Zweden heeft onder jullie aanmoediging, omdat ik toch wel erg moe was van het harde werken in de kliniek, geleid tot het kunnen schrijven van de introductie en de discussie van dit proefschrift, bedankt voor alles! 
Als laatste wil ik mijn allerliefste Mack bedanken. Lieve Siamack, bedankt voor je steun en aanmoediging bij eigenlijk alles wat ik onderneem of wil gaan ondernemen. Maar ook voor je begrip voor wanneer ik als echt avondmens midden in de nacht weer eens graag wilde praten over een moeilijke analyse, presentatie, nieuw idee, ofwel de feestlocatie voor mijn $\mathrm{PhD}$ receptie. Ik ben ervan overtuigd dat we elkaars positieve punten niet alleen op persoonlijk vlak maar ook op het gebied van werk versterken en dat is erg bijzonder. $\mathrm{Nu}$ jouw boekje nog en ondertussen, op naar onze bruiloft op 28 augustus! 
Curriculum Vitae 


\section{Curriculum Vitae}

Ellen Schoorel werd geboren op 13 augustus 1986 in Lelystad. In juni 2004 behaalde zij haar VWO diploma met profiel 'natuur en gezondheid' en Latijn aan de Scholen Gemeenschap Lelystad (SGL). In september 2004 startte zij aan de Universiteit Wageningen met de bachelor 'Nutrition and Health'. $\mathrm{Na}$ het succesvol afronden van deze bachelor werd zij geselecteerd voor de nieuwe research master 'Arts-Klinisch Onderzoeker' (A-KO). De A-KO opleiding leidt studenten op tot zowel arts als klinisch onderzoeker met als doel om een brug te slaan tussen klinisch onderzoek en de medische praktijk. In het laatste jaar van deze masteropleiding is zij betrokken geraakt bij de SIMPLE studie. In de tijdens dit jaar gevolgde afstudeerstage werd de basis gelegd voor het in dit proefschrift beschreven onderzoek. Na haar afstuderen als Arts-Klinisch Onderzoeker in augustus 2011 zette ze haar onderzoek dan ook voort in de vorm van een promotie traject onder supervisie van prof. dr. J.G. Nijhuis, dr. H.C.J. Scheepers en dr. R.P.M.G Hermens. Tijdens haar promotietraject publiceerde ze haar data in gerenommeerde internationale peer-reviewed medische tijdschriften en presenteerde zij haar data op diverse (internationale) conferenties. In april 2014 won ze de prijs voor 'Originele Studies' op de wetenschapsdag van het MUMC+.

Behalve als promovenda heeft ze de afgelopen jaren ook gewerkt als docent en als arts. Gedurende haar promotie werkte ze van september 2011 tot juni 2012 als docente op het Skillslab van Maastricht als docente medische basisvaardigheden. Van juni 2013 tot januari 2015 werkte ze als arts-assistent bij de interne geneeskunde in het MUMC+ om vervolgens in maart 2015 te starten met de huisartsopleiding in Maastricht. 


\title{
List of publications
}

\author{
Peer-reviewed publications \\ ENC Schoorel, S Melman, SMJ van Kuijk, WA Grobman, A Kwee, BWJ Mol, JG Nijhuis, LJM Smits, R \\ Aardenburg, K de Boer, FMC Delemarre, IM van Dooren, MTM Franssen, G Kleiverda, M Kaplan, SMI \\ Kuppens, FTH Lim, JM Sikkema, E Smid-Koopman, H Visser, FPJM Vrouenraets, M Woiski, RPMG Hermens, \\ HCJ Scheepers. Predicting successful intended vaginal delivery after previous caesarean section: external validation \\ of two predictive models in a Dutch nationwide registration-based cohort with a high intended vaginal delivery \\ rate. BJOG 2014; 121(7): 840-7.
}

ENC Schoorel, E Vankan, HCJ Scheepers, BCC Augustijn, CD Dirksen, M de Koning, SMJ van Kuijk, A Kwee, S Melman, JG Nijhuis, R Aardenburg, K de Boer, THM Hasaart, BWJ Mol, M Nieuwenhuijze, MG van Pampus, J van Roosmalen, FJME Roumen, R de Vries, MGAJ Wouters, T van der Weijden, RPMG Hermens. Involving women in personalised decision-making on mode of delivery after caesarean section: the development and pilot testing of a patient decision aid. BJOG 2014; 121(2): 202-9.

ENC Schoorel, SMJ van Kuijk, S Melman, JG Nijhuis, LJM Smits, R Aardenburg, K de Boer, FMC Delemarre, IM van Dooren, MTM Franssen, M Kaplan, G Kleiverda, SMI Kuppens, A Kwee, FTH Lim, BWJ Mol, FJME Roumen, JM Sikkema, E Smid-Koopman, H Visser, M Woiski, RPMG Hermens, HCJ Scheepers. Vaginal birth after a caesarean section: the development of a Western European population-based prediction model for deliveries at term. BJOG 2014; 121(2): 194-201.

ENC Schoorel, SMJ van Kuijk, JG Nijhuis, LJ Smits, HC Scheepers. Re: Predicting successful vaginal birth after Cesarean section using a model based on Cesarean scar features examined using transvaginal sonography. Ultrasound Obstet Gynecol 2013; 42(1): 122-3.

S Melman, ENC Schoorel, C Dirksen, A Kwee, L Smits, F de Boer, M Jonkers, MD Woiski, BW Mol, JP Doornbos, H Visser, AJ Huisjes, MM Porath, FM Delemarre, SM Kuppens, R Aardenburg, IM Van Dooren, FP Vrouenraets, FT Lim, G Kleiverda, PC van der Salm, K de Boer, MJ Sikkema, JG Nijhuis, RP Hermens, HC Scheepers SIMPLE: implementation of recommendations from international evidence-based guidelines on caesarean sections in the Netherlands. Protocol for a controlled before and after study. Implement Sci 2013; 8: 3.

ENC Schoorel, HCJ Scheepers, JG Nijhuis. Pregnancy and delivery after prior caesarean section: shared decision making and be aware of signs of (imminent) uterine rupture. NTOG, 2012; 125: 384-389.

\section{Submitted for publication}

S Melman, ENC Schoorel, K de Boer, H Burggraaf, JB Derks, D van Dijk, J van Dillen, CD Dirksen, JJ Duvekot, A Franx, THM Hasaart, AJM Huisjes, D Kolkman, S. van Kuijk, A Kwee, BW Mol, MG van Pampus, A de RoonImmerzeel, JJM van Roosmalen, FJME Roumen, E Smid-Koopman, L Smits, WA Spaans, H Visser, WJ van Wijngaarden, C Willekes, MGAJ Wouters, JG Nijhuis, RPMG Hermens, HCJ Scheepers. Development and measurement of guidelines-based quality indicators of caesarean section care in the Netherlands: a RANDmodified Delphi procedure and retrospective medical chart review.

ENC Schoorel, E Vankan, SM van Kuijk, BWJ Mol, JG Nijhuis, R Aardenburg, K de Boer, FMC Delemarre, CD Dirksen, IM van Dooren, MTM Franssen, M Kaplan, G Kleiverda, SMI Kuppens, A Kwee, FTH Lim MD, S Melman, MM Sikkema, E Smid-Koopman, LJ Smits, H Visser, J Langenveld, M Woiski, HC Scheepers, RP Hermens. Practice variation of vaginal birth after caesarean: a registration based cohort study.

ENC Schoorel, DRM Hünen, SMJ van Kuijk, BCC Augustijn, A Kwee, BWJ Mol, JG Nijhuis, LJM Smits, RPMG Hermens, HCJ Scheepers. Probability of vaginal birth after caesarean: do not ask the obstetrician. 